\title{
Exploring for graphite using a new ground-based time-domain electromagnetic system
}

\author{
By
}

Eric Meunier

\begin{abstract}
A thesis submitted to the Faculty of Graduate and Post-Doctoral Affairs in partial fulfillment of the requirements for the degree of
\end{abstract}

Master of Science

in

Earth Sciences

\author{
Carleton University \\ Ottawa, Ontario
}

(C) 2015

Eric Meunier 


\section{Abstract}

With the proliferation of battery-powered technology, such as cell phones, laptops, and electric cars, the need for graphite is expected to increase as it plays a crucial role in batteries and fuel cells. The mineral is already used for many applications where its unique properties make it difficult or sometimes impossible to replace. As the value of graphite increases, small graphite deposits that were not economically feasible in the past could become viable. Their small size requires exploration methods with high spatial resolution able to accurately detect and delineate the deposits. We present the IMAGEM system, a high-resolution ground-based time-domain electromagnetic system, whose high spatial resolution allows it to accurately delineate small deposits, on the order of a few metres thick. We perform forward modelling and inversion using a 1D modelling software program, AarhusInv, and present the results of case studies where the system was used for graphite exploration. The IMAGEM system was able to differentiate multiple distinct anomalies where the classic MaxMin system could only outline one anomaly. The IMAGEM system is still being developed and shows great potential for detecting small near-surface (depth less than $\sim 20 \mathrm{~m}$ ) highly-conductive bodies, such as graphite deposits. AarhusInv provides great flexibility in system configuration, and proves to be an effective program to model the IMAGEM system. 


\section{Acknowledgments}

I would like to thank Dr. Claire Samson, my thesis supervisor, for helping and guiding me through this project. Without her encouragement and support, this project would have never occurred.

I'd like to express my heartfelt thanks to Jean-Christophe Ricard, president of Devbrio Géophysique Inc. for having given me the opportunity to use his survey system and for taking time out of his very busy schedule to help me, and to Andrew Hay, project manager for Devbrio Géophysique Inc., for always being willing to help.

I would also like to thank Ray Caron (Ph.D. candidate) and Michael Cunningham (M.Sc. student) for their help early on in my research, and to Jennifer Blanchard (M. Sc. student) for her support and willingness to help. 


\section{Table of Contents}

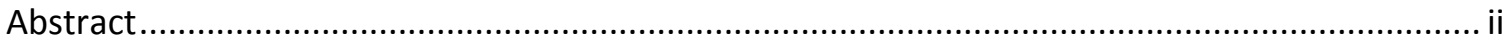

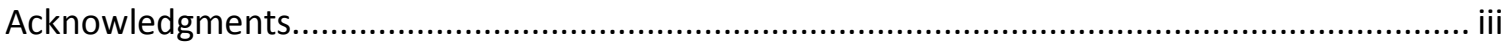

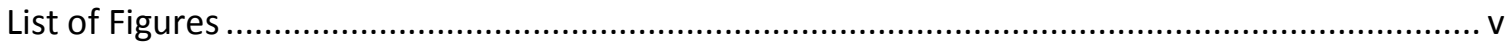

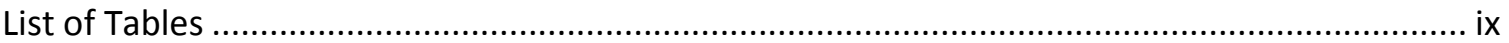

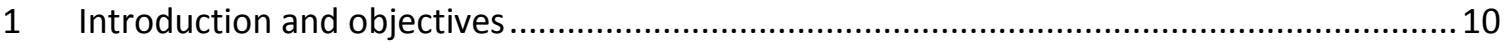

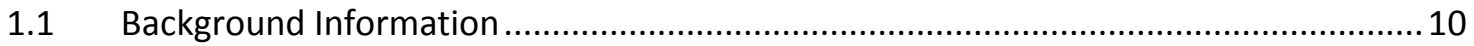

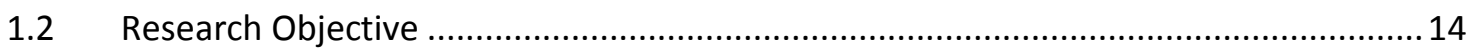

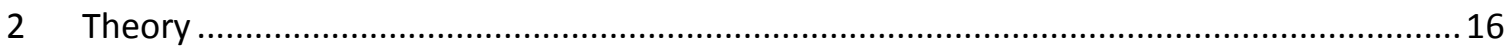

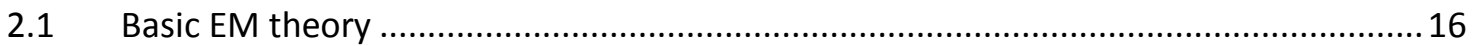

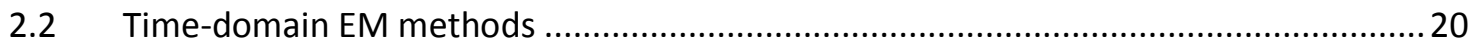

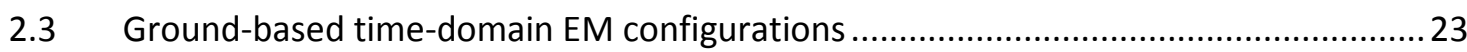

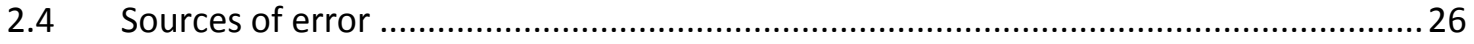

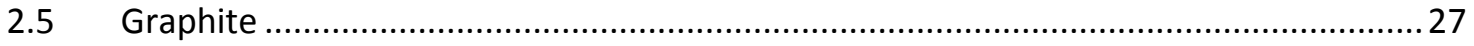

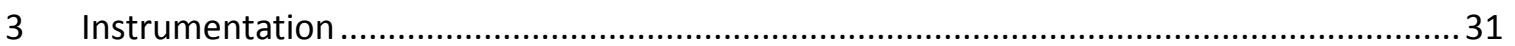

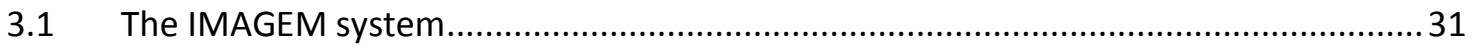

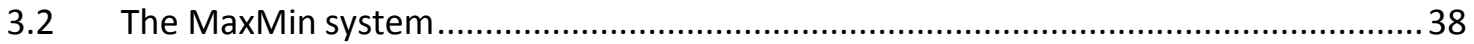

3.3 Comparing the IMAGEM system and the MaxMin I-8 system .................................42

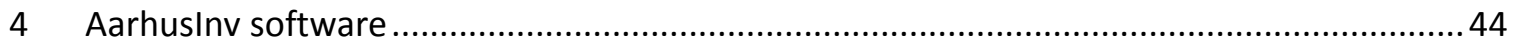

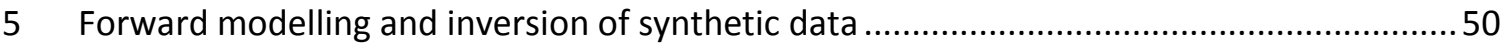

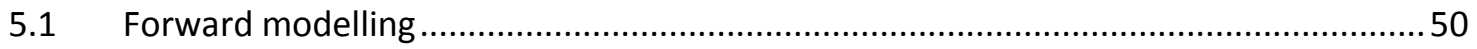

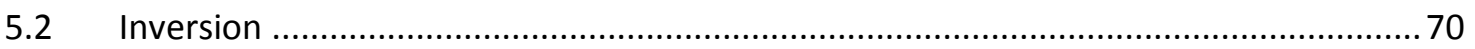

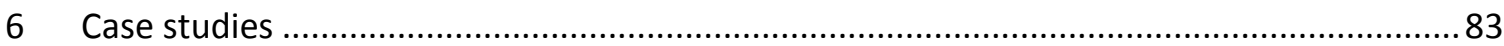

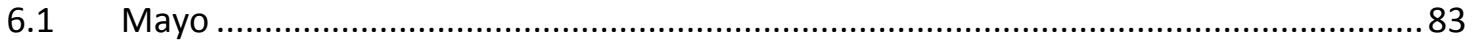

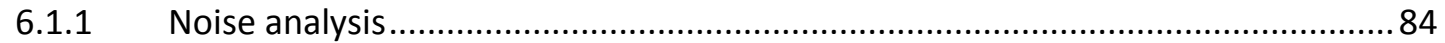

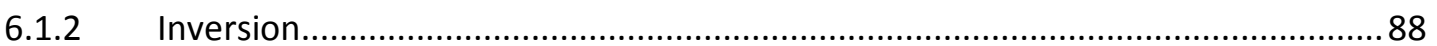

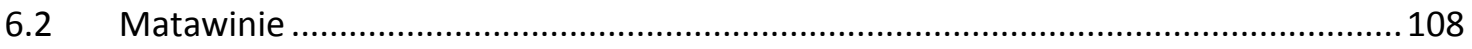

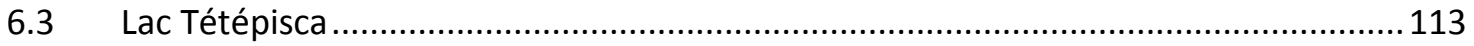

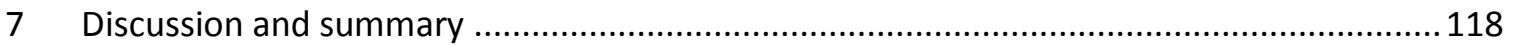

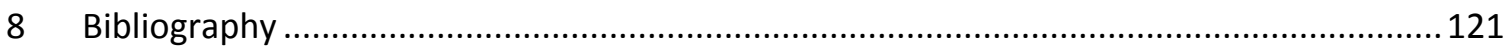




\section{List of Figures}

Figure 2.1. Representation of the basic principles of EM sounding methods (modified from Grant \& West 1965)

Figure 2.2. Representation of the current induced in the ground as the transmitter is turned off. As the transmitter is turned off, the current induced in the ground is mirroring the transmitter loop near the surface. As time after turn-off increases, the current propagates through the ground and decays in amplitude.

Figure 2.3. Common ground-based EM system configurations (from Nabighian \& Macnae, 1991).

Figure 3.1. The IMAGEM system. [A] View of the front operator. The black box attached to the operator contains the tablet computer and other electronic hardware. The operator wears a harness that is attached to the frame of the system so the system can be carried hands-free. [B] View of the side of the system. The orange box contains the battery and other electronic hardware. The white box in the centre of the system covers the dipole receiver (Photos courtesy of Devbrio Géophysique Inc.).

Figure 3.2. (A) Two full cycles of the IMAGEM transmitter. One cycle consists of a positive pulse and one negative pulse. (B) The half-sine pulse waveform used by the IMAGEM system (Modified from Ricard, 2013).

Figure 3.3. The MaxMin I-8 system. [A] The receiver (foreground) and transmitter (background) are connected by a cable. [B] The transmitter loop. [C] The receiver coils, positioned vertically for the HCP configuration (Photos courtesy of Devbrio Géophysique Inc.) .................................40 Figure 3.4. Common transmitter-receiver configurations for FDEM systems. $\mathrm{HCP}=$ Horizontal coplanar. PERP=Perpendicular. VCP=Vertical coplanar. VCA=Vertical coaxial. The MaxMin system most commonly uses the HCP configuration, but can also employ the PERP and VCP configurations (Nabighian \& Macnae, 1991).

Figure 4.1. The MOD file contains the information about the initial guess model and some inversion parameters. A constraint mode of 0 means no constraint is applied. Inversion.tem is the TEM file the program will use. Prior STD is the factor within which the parameters may vary.

The value of -1 means the parameters are free to vary. 46

Figure 4.2. The TEM file contains the information about the system and the data to invert. In this example, the ' 7 ' on line 2 represents a rectangular loop transmitter and the ' 3 ' a Z-direction magnetic field receiver. The values on line 3 represent the position of the transmitter and receiver. In this case, both are coincident and centered (in-loop configuration). The three values of ' 3 ' on line 5 means the data is input, transformed, and output as $d B / d t\left(V / m^{2}\right)$. Line 6 states the waveform is user-defined; it is then defined in line 7. Finally, starting from line 9 and on, the data is input, starting with the channel centre time on the left, followed by the value of the response at that channel, then the standard deviation of the data value, then which waveform is used and which low pass filter is used. In this case, no filter is used.

Figure 4.3. The EMO file, which is the output file after an inversion or forward model. In this example, the results of an inversion are presented. The bottom half of the figure, under 
'Parameters', shows the resulting inverted model, including values of resistivity and thickness for every iteration from the initial guess model to the final inverted model.

Figure 4.4. Representation of the IMAGEM's configuration used for modelling with AarhusInv. The red square represents the transmitter loop. The blue circle represents the receiver dipole.49 Figure 5.1. The forward modelling and inversion process for synthetic data. The black boxes represent data products, and white boxes represent operations

Figure 5.2. Visual representation of the half-space models. [A] $1 \Omega \cdot m$ half-space. [B] $100 \Omega \cdot m$ half-space.

Figure 5.3. Visual representation of the overburden resistivity models, where only the resistivity of the overburden layer is varied. [A] consists of a $10 \mathrm{~m}$ thick overburden layer with a resistivity of $1 \Omega \cdot m$ overlying a $1000 \Omega \cdot m$ half-space. [B] consists of a $10 \mathrm{~m}$ thick overburden layer with a resistivity of $100 \Omega \cdot m$ overlying a $1000 \Omega \cdot m$ half-space.

Figure 5.4. Visual representation of the overburden thickness models, where only the thickness of the overburden layer is varied. [A] consists of a $5 \mathrm{~m}$ thick overburden layer with a resistivity of $10 \Omega \cdot m$ overlying a $1000 \Omega \cdot m$ half-space. [B] consists of a $20 \mathrm{~m}$ thick overburden layer with a resistivity of $10 \Omega \cdot m$ overlying a $1000 \Omega \cdot m$ half-space

Figure 5.5. Synthetic decay curves (no noise added) generated by forward modelling the halfspace models (red), overburden resistivity models (green), and overburden thickness models (blue).

Figure 5.6. Response of multiple channels for graphite model A. Every 5 models shares the same graphite layer depth, but with increasing graphite layer thickness. A summary of the depth and thickness of the graphite layer represented on the abscissa is presented in Table 5.2. The model resistivity used is presented in Table 5.3.

Figure 5.7. Response of multiple channels for graphite model B. Every 5 models shares the same graphite layer depth, but with increasing graphite layer thickness. A summary of the depth and thickness of the graphite layer represented on the abscissa is presented in Table 5.2. The model resistivity used is presented in Table 5.3.

Figure 5.8. Response of multiple channels for graphite model C. Every 5 models shares the same graphite layer depth, but with increasing graphite layer thickness. A summary of the depth and thickness of the graphite layer represented on the abscissa is presented in Table 5.2. The model resistivity used is presented in Table 5.3.

Figure 5.9. Contour plot of the response of model A versus the depth and thickness of the graphite layer. 66

Figure 5.10. Contour plot of the response of model $\mathrm{C}$ versus the depth and thickness of the graphite layer.

Figure 5.11. The forward model decay curves with added synthetic noise. The synthetic noise is based on calculated noise measured with the IMAGEM system. .71

Figure 5.12. Result of the inversion of the half-space models. [A] Inversion of half-space model with a resistivity of $1 \Omega \cdot \mathrm{m}$. [B] Inversion of half-space with a resistivity of $100 \Omega \cdot \mathrm{m}$

Figure 5.13. Comparing the decay curve achieved by the forward model with the decay curve achieved by forward modelling of the inverted model for the half-space models. 
Figure 5.14. Result of the inversion of the overburden resistivity models. [A] Inversion of the 1 $\Omega \cdot \mathrm{m}$ overburden model over a $1,000 \Omega \cdot \mathrm{m}$ half-space. [B] Inversion of the $100 \Omega \cdot \mathrm{m}$ overburden model over a $1,000 \Omega \cdot m$ half-space.

Figure 5.15. Comparing the decay curves calculated using the initial model (solid lines) with the decay curves calculated by forward modelling of the inverted model (dotted lines) for the overburden resistivity models.

Figure 5.16. Result of the inversion of the overburden thickness models. [A] Inversion of the $5 \mathrm{~m}$ overburden model with a resistivity of $10 \Omega \cdot m$ over a $1,000 \Omega \cdot m$ half-space. [B] Inversion of the $20 \mathrm{~m}$ thick overburden model over a 1,000 $\Omega \cdot \mathrm{m}$ half-space.

Figure 5.17. Comparing the decay curve achieved by the forward model with the decay curve achieved by forward modelling the inverted model for the overburden thickness models.

Figure 6.1. Response of the IMAGEM system for a $1.1 \mathrm{~km}$ long section of a survey line at the Mayo calibration site. The red rectangle represents the section used for the noise analysis......85 Figure 6.2. Response of the IMAGEM system for the stack of measurements enclosed in the red rectangle in Figure 6.1.

Figure 6.3. (Top) Stack of the decay curves displayed in Figure 6.2. (Bottom) Standard deviation versus channel number. The mean standard deviation (dashed-dotted line) across each channel is $2.96 \times 10^{-8} \mathrm{~V} / \mathrm{m}^{2}$.

Figure 6.4. Results of the MaxMin survey data.

Figure 6.5. Inversion results using model 1 . The dotted blue line on the second graph from the top represents the shallowest depth of penetration of the MaxMin system for the configuration used during the survey.

Figure 6.6. Inversion results using model 2. The dotted blue line on the second graph from the top represents the shallowest depth of penetration of the MaxMin system for the configuration

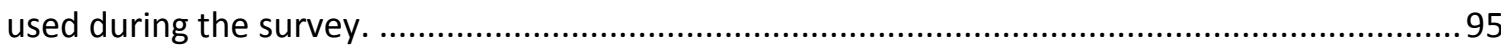

Figure 6.7. Inversion results using model 3.............................................................................96

Figure 6.8. Decay curves of points A, B, and C of Figure 6.1. The gaps observed in curve B is caused by negative values which have been removed from analysis. ........................................98

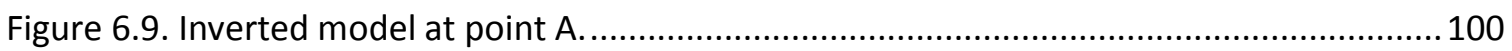

Figure 6.10. Comparing the IMAGEM field decay curve with the decay curve achieved by forward

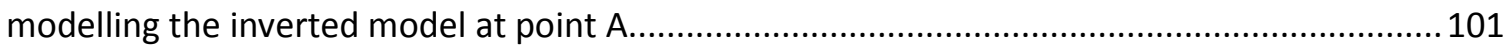

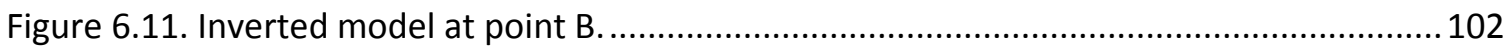

Figure 6.12. Comparing the IMAGEM field decay curve with the decay curve achieved by forward

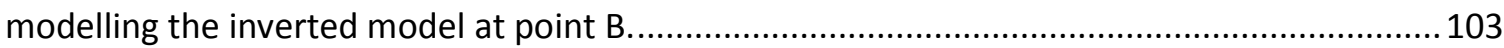

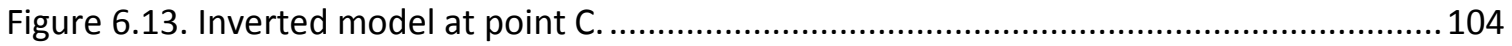

Figure 6.14. Comparing the IMAGEM field decay curve with the decay curve achieved by forward modelling the inverted model at point $\mathrm{C}$. 105 The anomalies observed are approximately on the order of 1-100 metres in size. The scattered nature observed would suggest that the deposit is formed of many small clusters of graphite. Figure 6.15. Local geology of the Matawinie survey area (source: Geological Survey of Quebec). 
Figure 6.16. Interpolated results of the Matawinie survey for channel 50 using Inversed Distance Weighing (IDW). (Data source: Nouveau Monde Mining Inc., shown with permission).

Figure 6.17. Visualizing survey data at increasing depth using the interpolated maps at increasing channel times.

Figure 6.18. Digital elevation model (DEM) and survey lines for the northern part of the survey

area (Ricard et al., 2015). Arrows point to the line presented in Figure 6.19. 115

Figure 6.19. IMAGEM data results. Amplitude of channel 50 (0.3 ms after turn-off) color coded against elevation contours (Ricard et al., 2015). Arrows point to the line presented in Figure 6.18

(Data source: Focus Graphite Inc., shown with permission) 116

Figure 6.20. IMAGEM data for channels 45 to 90 ( $0.21 \mathrm{~ms}$ to $4.3 \mathrm{~ms}$ after turn off; top panel). MaxMin data at a frequency of $3520 \mathrm{~Hz}$ (middle panel: solid and dotted lines are the in-phase and quadrature components, respectively), and elevation profile (bottom panel) (Ricard et al., 2015). 


\section{List of Tables}

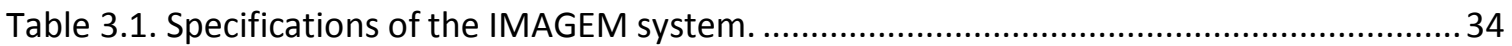

Table 3.2. Times after turn-off for the 110 channels of the IMAGEM system. 'Tx': transmitter. . 36

Table 5.1. Summary of the model parameters used for forward modelling and inversion using

AarhusInv.

Table 5.2. Summary of the different models analyzed with the depth and thickness of the graphite layer

Table 5.3. Resistivity values for the three layers of the graphite models. Layer 2 represents the graphite layer

Table 5.4. Summary of the results of inverting synthetic data................................................. 72

Table 6.1. The different initial guess models used for the inversion of the MaxMin data............90

Table 6.2. Summary of the results of inverting field data with the IMAGEM system. 


\section{Introduction and objectives}

\subsection{Background Information}

Geophysics is a discipline of applied physics used to obtain information about the subsurface indirectly. More precisely, it is the use of a set of measurements of a physical field made on the earth's surface to obtain information about the interior spatial distribution of one or more of the earth's physical properties. Exploration geophysics is a branch of geophysics used to detect or infer the presence and position of economical ore bodies, hydrocarbons, groundwater reservoirs, and other geological structures.

There are several different methods of exploration geophysics, which either measure different physical properties, or use different approaches to measure the same physical property. For example, seismic surveys measure the velocity of the propagation of seismic waves in the earth, magnetic surveys measure the magnetic intensity of the geomagnetic field, electrical surveys measure the conductivity of the subsurface, and gravity surveys measure the relative gravitational acceleration at different locations. These are all different methods of geophysics which measure different properties of the subsurface. An example of different geophysical methods which measure the same physical property is electromagnetic methods and direct-current resistivity methods. These two methods measure the resistivity of the subsurface, but do so using different instruments and procedures. 
Electromagnetic (EM) methods use time-varying electrical current to induce magnetic fields, which are used to indirectly measure the conductivity of the subsurface. They are used in a variety of applications such as:

- Mineral exploration: metallic elements found in certain deposits create a highly conductive anomaly.

- Groundwater investigations: contaminant ions such as salts and acids increase the conductivity of the groundwater.

- Mapping: different rocks have different ranges of conductivities which can be used for stratigraphic mapping.

- Geothermal reservoirs: the alteration of host rock by hydrothermal fluids can increase the conductivity of the host rock.

- Environmental: EM methods can be used to locate hazards such as contaminants and old military devices, like unexploded ordnances (UXO) and landmines.

Electromagnetic methods have certain advantages over other geophysical techniques. They require no direct electrical contact with the ground, allowing for surveys to be conducted effectively in frozen environments and from aircraft. However, they do not work well in highly resistive environments and they are also susceptible to interference from cultural sources such as power lines. Data processing requires sophisticated analytical and interpretation skills. 
There are two types of electromagnetic methods: Frequency-Domain Electromagnetics (FDEM) and Time-Domain Electromagnetics (TDEM), also known as Transient EM (TEM) methods. In FDEM methods, the ground is energized by passing an alternating current (AC) through a conductive, metallic wire (transmitter). This creates a primary field which induces eddy currents in conductive material in the subsurface. These eddy currents in turn induce a secondary electromagnetic field, which is recorded in another conductive wire (receiver). The secondary field contains the information about the conductors in the subsurface and is many orders of magnitude smaller than the primary field. Because the receiver will record both the primary and secondary field concurrently, it is difficult to isolate the secondary field. This led to the development of TDEM methods. In TDEM methods, a direct current (DC) is usually passed through a transmitter loop and abruptly shut off. The receiver can then measure the decay of the secondary field without the presence of the primary field.

The use of TDEM methods to survey a massive sulphide ore body was first disclosed in 1951, and a patent application was filed in 1953 by Newmont Exploration Ltd. They later developed the Newmont EMP system in 1962, which consisted of a large transmitter loop and moving receivers and used ninety $12 \mathrm{~V}$ car batteries to supply a $700 \mathrm{~A}$ pulse for a duration of $100 \mathrm{~ms}$. The receivers consisted of a coil with an effective area of 20,000 turns $-\mathrm{m}^{2}$. In 1958 , the first airborne TDEM system was developed by Barringer, called the INPUT system. The horizontal transmitter loop, using half-sine pulses, was placed around the aircraft and the receiver was towed in a 'bird' behind the plane. The advent of computer technology and introduction of many new electronic 
components in the 1970 s led to a revolution in TDEM methods (Nabighian \& Macnae, 1991). In 1974, Newmont Exploration Ltd. created the first high-power, threecomponent, fully digital TDEM system. The 1970s and 1980s saw the introduction of many other systems, including SIROTEM, Crone PEM, UTEM 1, Geonics EM-37, GEOTEM, and PROSPECT. Multipurpose systems, which can acquire data from multiple geophysical methods, made their appearance during this period. By 1988, all manufacturers offered fully digital TDEM systems (Nabighian \& Macnae, 1991)

At least from a business standpoint, one of the most important aspects of geophysics is inversion. The process of converting field measurements into a physical property distribution is termed 'modelling' or 'interpretation'. One of the revolutionary progressions in exploration geophysics due to the advent of computer technology is the use of computers to generate plausible models of the subsurface based on field data. This specific process is termed 'inversion'. Geophysical inversion is challenging and requires user insight even in the best circumstances. For example, the shape of a given EM anomaly could be fitted using a thin-sheet model with a certain thickness and conductivity. This process, however, does not yield a unique solution. The EM response is a combination of the thickness, depth, and conductivity, and so by varying these parameters, a similar response can be achieved with different thin-sheet models. This ambiguity is inherent to the inversion process. Although the basic laws that relate the EM field and the physical property distribution are well known (Maxwell's equations), we still lack the capability to model the response created by a moderately complex physical distribution models. Computer modelling uses very simple geometric shapes 
like planar horizontal layers to represent the Earth. However, realistic geological scenarios are varied and very complex, and few can accurately be described using simple geometric shapes. For this reason, human interpretation remains necessary to guide the modelling process.

\subsection{Research Objective}

The research project presented in this thesis concerns principally the IMAGEM system. The IMAGEM system (Ricard, 2013) is a new ground-based TDEM system which uses a small transmitter loop with an unorthodox shape and operates at a high frequency allowing for continuous recording. It has been designed to perform rapid surveys with high spatial-resolution mainly for shallow and high-conductivity targets, such as graphite deposits. The system uses an 'in-loop' configuration (Section 2.3), and is operated either by two persons on foot or towed by vehicle. The unique transmitter shape and size creates difficulty for modelling the response of the system and inverting field data. To be able to accurately model the IMAGEM system, a software program with high flexibility in system configuration is needed. AarhusInv (Auken et al., 2014) is a software program for the forward modelling and inversion of electrical and electromagnetic geophysical data. It can model any geometric system shape, which is ideal for the IMAGEM system.

The objective of this thesis is to demonstrate the survey capabilities of the IMAGEM system to detect and delineate graphite deposits. It will be compared to the MaxMin system (Apex Parametrics Limited, n.d.), an established FDEM system. 
Chapter 2 explains the basic theory of electromagnetic survey methods, and provides information about graphite, including how it is formed, how it can be detected, and why it is an important strategic mineral. Further information about the two survey systems is presented in Chapter 3, including a comparison between the two. The software program is described in Chapter 4, and this chapter also includes information about how to use it. Chapter 5 presents the results of forward modelling and inversion of synthetic data, specifically tailored for the IMAGEM system. Three case studies are presented in Chapter 6 to showcase the capabilities of the system in respect to graphite exploration. 


\section{Theory}

\subsection{Basic EM theory}

Light, radio, radar, microwaves, $\mathrm{X}$-rays, and gamma rays are all examples of electromagnetic waves, which are produced by the acceleration of electrons or other charged particles. As the name suggests, an electromagnetic field has two components perpendicular to each other: the electric field and the magnetic field. The two fields can be described in terms of the field intensity vectors, $E(V / m)$ and $H(A / m)$, or in terms of the flux density vectors, $\mathrm{D}\left(\mathrm{C} / \mathrm{m}^{2}\right)$ and $\mathrm{B}\left(\mathrm{Wb} / \mathrm{m}^{2}\right.$ or Tesla). These fields are functions of spatial position $r(x, y, z)$, and time $t$ (seconds) or frequency $f$ (hertz).

The propagation of electromagnetic waves is a function of the electrical properties of the material through which the current flows. These properties affect the velocity of the wave, the efficiency of propagation, and the energy lost within the medium (Burger et al., 2006).

Electrical resistivity $\rho$ and its reciprocal electrical conductivity $\sigma$ are physical properties of material that describe the mobility of an electric charge in the presence of an electric field. Electric charge is impeded and does not propagate efficiently in resistive material, and moves freely in conductive material. By Ohm's law, resistance $R$ is given by

$$
R=V / I
$$

where $V$ is the potential difference and $/$ is current. The physical property of resistivity can be determined from 


$$
\rho=R A / l
$$

where $A$ is the cross-sectional area and / is the length of the resistor. Resistivity is measured in units of $\Omega \cdot \mathrm{m}(\mathrm{Ohm} \cdot \mathrm{metre})$, and conductivity by $\mathrm{S} / \mathrm{m}$ (Siemens/metre).

Electric charges in motion (a current) generate a magnetic field. Electric charges can move as free electrons or as mobile ions, and can also move in a conductive medium without affecting the charge balance of the medium. Current flow can be described by the electric current flux density vector $J\left(A / \mathrm{m}^{2}\right)$. According to Ampère's law, the magnetic field generated by a current circulates around J. The density of current in a medium created by an electric field is linearly proportional to the electric field strength according to Ohm's law

$$
J=\sigma E
$$

The coupling between the $E$ and $H$ fields is described by Ampère's law and Faraday's law. Each field generates a component of the other field: an electric field is created by a time-varying magnetic field and a magnetic field is created by a timevarying electric field

$$
\nabla \times E=-\frac{\partial B}{\partial t}
$$

and

$$
\nabla \times H=\frac{\partial D}{\partial t}+J
$$

EM methods measure the conductivity of the subsurface, and are more sensitive to conductive bodies than resistors (Burger et al., 2006). EM soundings operate on the 
principle of electromagnetic induction: as per Ampère's law, a primary magnetic field $B$ is produced by the transmission of an electrical current / through a conductive coil (the transmitter). The strength of the magnetic field is proportional to the strength of the electric current and has the same phase. As per Faraday's law, the primary field, which is time-varying, will in turn induce electrical eddy currents in conductors in the ground. The strength of the induced electric current is proportional to the rate of change $(d B / d t)$ of the magnetic field. These eddy currents will generate a secondary magnetic field, which is also time-varying, and will be measured by the receiver, which consists of another conductive coil (Figure 2.1). 


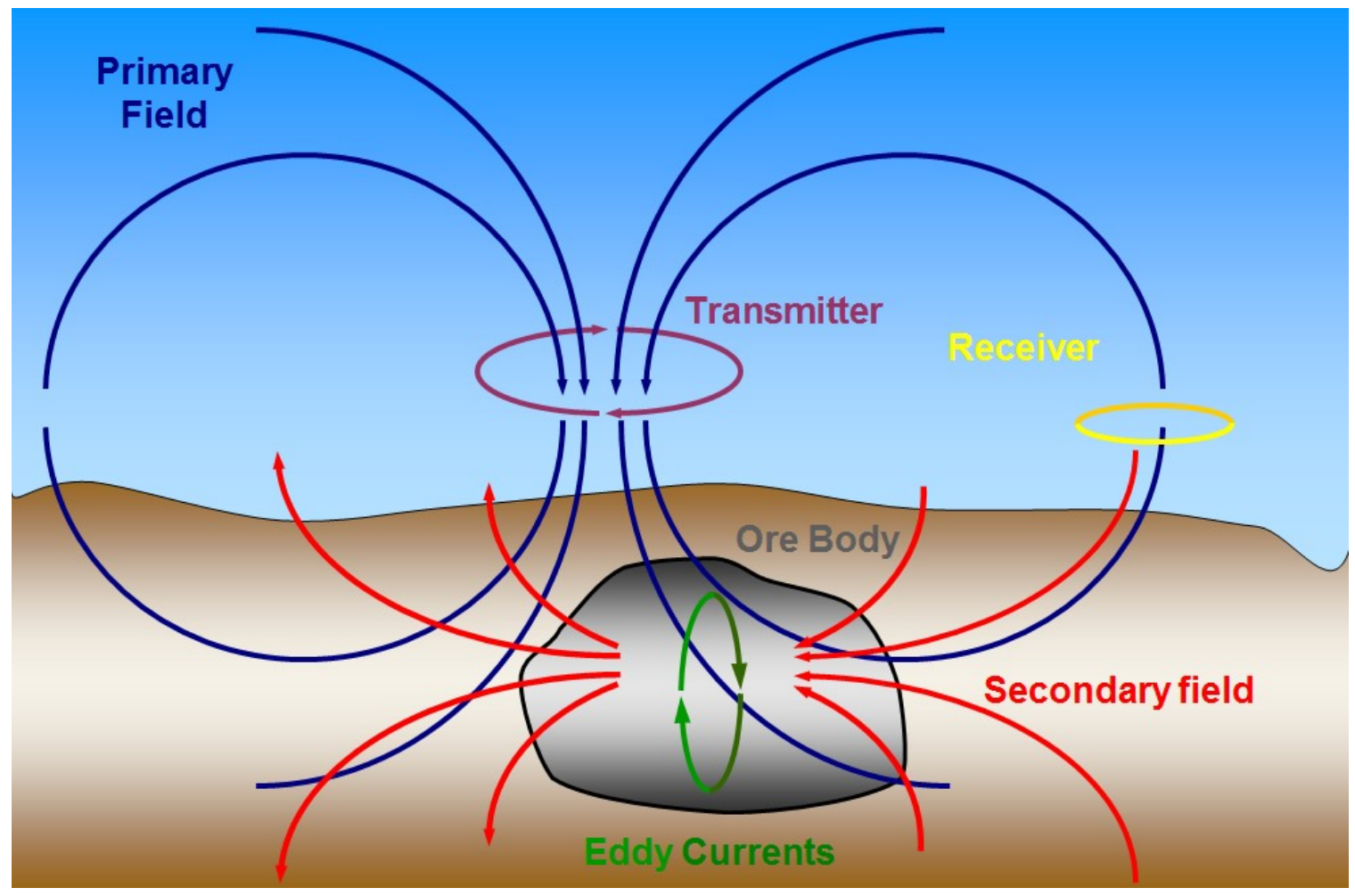

Figure 2.1. Representation of the basic principles of EM sounding methods (modified from Grant \& West 1965). 


\subsection{Time-domain EM methods}

In time-domain electromagnetic methods, a primary field is generated by an electric current in a conductive coil (transmitter) for a finite length of time and abruptly turned off. The length of time when the current is circulating through the transmitter is known as the 'on-time pulse', and the process of shutting it off rapidly is called a 'step function excitation'. According to Faraday's law, the process of rapidly setting the current to zero induces a short-duration magnetic field (primary field), which in turn, induces a short current pulse in the ground in the vicinity of the transmitter loop. This current immediately begins to decay because of the finite resistivity of the ground (Figure 2.2). With highly conductive bodies, the current decays more slowly than for weak conductors. This time-varying current produces a secondary magnetic field, whose decay induces in turn electric currents in nearby conductors, including the receiver, which is often located in the centre of the transmitter coil. The measurements of the decaying secondary magnetic field begin immediately after turn-off, and measurements are made in rapid succession (at different time gates, or 'channels') until the next ontime pulse. Due to the skin effect, at early times in the measurement of the signal, electric currents induced by the time-varying magnetic field in the ground are concentrated near the surface. As time increases, the current propagates to greater depths, and the measured signal will reflect the resistivity of the deeper layers.

The rate of change of currents induced in the ground and their associated magnetic field is a function of the conductivity, size, and shape of the conductive 
material. For a step function excitation of a transmitter loop, the current flowing in a conductive body as a function of time $t$ is (Nabighian \& Macnae, 1991):

$$
I(t)=\frac{M_{01} I_{0}}{L} e^{-t / \tau}
$$

where $M_{01}$ is the mutual inductance between the transmitter and the conductive body, $I_{0}$ is the transmitter current, $R$ and $L$ is the resistance and inductance of the conductive body, and $\tau=L / R$ is the time constant of the circuit. This equation states that the sudden interruption of the transmitter current instantaneously creates a current in the underground circuit which decays exponentially with a time constant $\tau$. At time $t=0$, the amplitude of the current is a function of the primary field flux $\left(M_{01} I_{0}\right)$ and conductor size (L), but independent of resistance $(R)$. The time constant $\tau$ is a function of both conductance and size (Nabighian \& Macnae, 1991). 


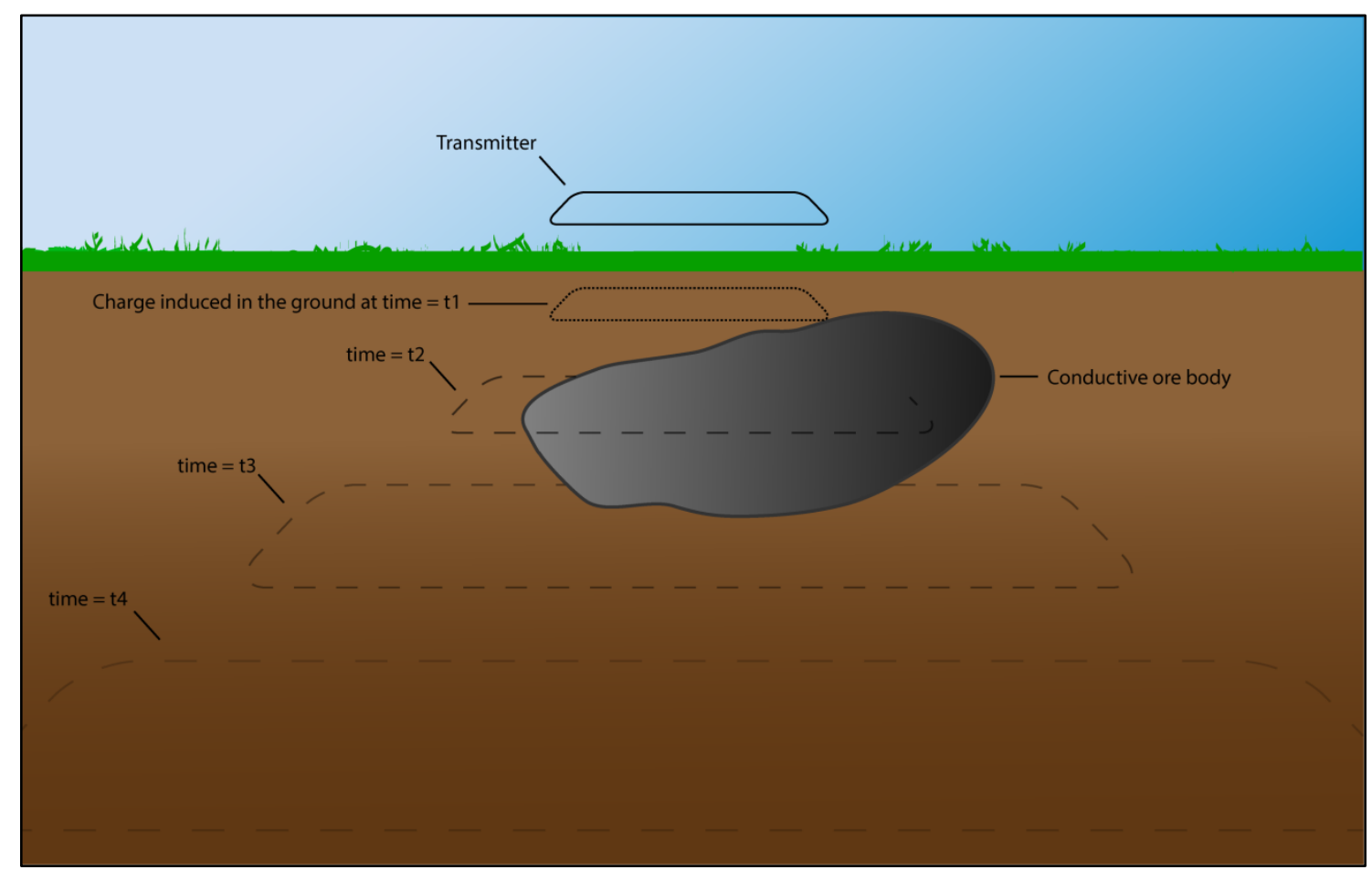

Figure 2.2. Representation of the current induced in the ground as the transmitter is turned off. As the transmitter is turned off, the current induced in the ground is mirroring the transmitter loop near the surface. As time after turn-off increases, the current propagates through the ground and decays in amplitude. 


\subsection{Ground-based time-domain EM configurations}

The most common ground-based TDEM system configuration consists of a single turn loop square transmitter, usually on the order of $10 \mathrm{~m} \times 10 \mathrm{~m}$ to $100 \mathrm{~m} \times 100 \mathrm{~m}$, with a smaller multi-turn horizontal receiver coil located in the centre of the transmitter. However, there are many different types of configurations:

- Single loop transmitter and receiver: In this configuration, a single loop is used as both the transmitter and the receiver (Figure 2.3a).

- Coincident transmitter-receiver loop: The transmitter and receiver are laid out spatially parallel, with the receiver positioned inside the transmitter loop (Figure 2.3b).

- In-loop (central loop) configuration: This configuration is similar to the coincident loop configuration but the receiver is a smaller multi-turn dipole receiver located at the centre of the transmitter loop (Figure 2.3b).

- Separated loop (slingram array): The transmitter and receiver are separated by a fixed distance (Figure 2.3c).

- Dual loop configuration: The transmitter and receiver form two loops connected in parallel (Figure 2.3e).

- Fixed transmitter: A large stationary transmitter loop is laid down, and the small receiver is moved along the survey path (Figure 2.3f). The transmitter loop can range in size from a few hundred metres to a few kilometres. 
The IMAGEM system is an in-loop system. The advantages of this include the system being more compact and interpretation being more intuitive than when the transmitter and receiver are separated by a distance. 

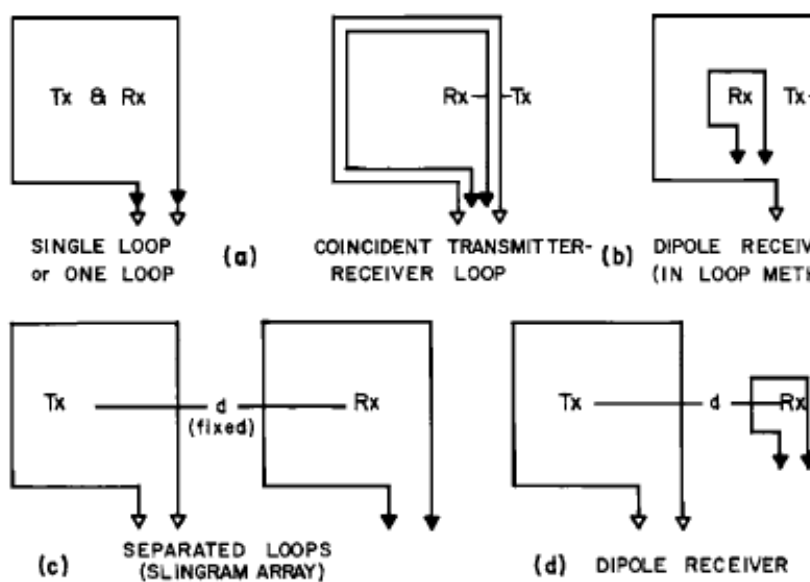

(c)

(SLINGRAM ARRAY)

LARGE LOOP RECEIVER

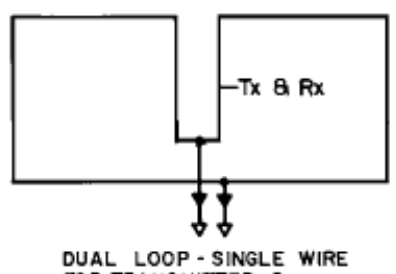

DUAL LOOP - SINGLE WIRE FOR TRANSMITTER

(e)

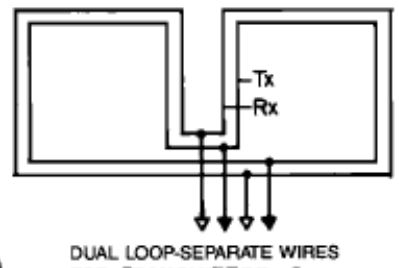

RECEIVE

DUAL LOOP-SEPARATE WIRES

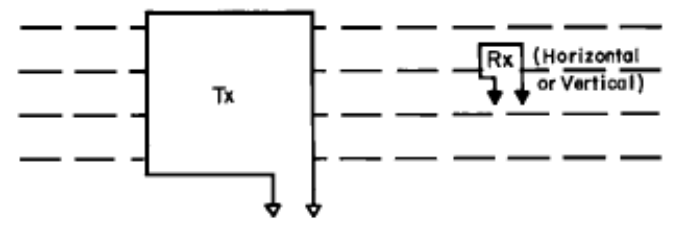

(f) LARGE FIXED TRANSMITTER LOOP

WITH ROVING RECEIVER.

Figure 2.3. Common ground-based EM system configurations (from Nabighian \& Macnae, 1991). 


\subsection{Sources of error}

There are many sources of errors that can affect the measurements of a TDEM system. These sources can be categorized in three categories: geometric sources, cultural sources, and electromagnetic noise.

- Geometric sources of error are caused by the change in the geometric relationship between the transmitter and receiver. It also includes topographic effects. In conductive environments, topography can cause significant coupling errors and must be corrected for.

- Cultural errors are caused by man-made metallic objects such as pipelines, metallic fences, etc. which are very conductive and can produce spurious anomalies.

- Electromagnetic noise can be caused by several sources:

- Geomagnetic noise is caused by natural EM transients generated by lightning discharges.

- Motion induced noise or microphonics is noise caused by the movement of the magnetic field sensors in the Earth's magnetic field. This is also called 'wind noise' and is more significant in open areas and in airborne surveys.

- Electric power distribution grids (60 Hz in North America) and Very Low Frequency (VLF) radio stations are the main sources of man-made electromagnetic noise. 
To increase the signal to noise $(\mathrm{S} / \mathrm{N})$ ratio, almost all TEM systems use a repetitive signal and stacking, which is the process of taking the average of multiple measurements at the same location.

\subsection{Graphite}

Graphite is a naturally occurring mineral composed of the element carbon. It is found in virtually every country in the world. However, most graphite deposits are not economically viable. As of 2012, the top producers of natural graphite in the world are China, with an estimated $67 \%$ of the global production, followed by India with $13 \%$, then Brazil, North Korea, Canada, and Russia, in decreasing order of tonnage produced. Combined, these six countries are accountable for $96 \%$ of the world's production (Olson, 2012). In 2012, Canada was the leading country for natural graphite development with a favorable outlook for new mines (Olson, 2012). Worldwide demand for graphite steadily increased throughout 2012 to 2014 (U.S. Department of the Interior, 2015). Applications in the high-tech sector, such as batteries for laptops, cell phones, and electric fuel cells, are driving the demand. For many countries, like the United States for example, graphite is deemed a strategic mineral, as it is important to the nation's economy, doesn't have many replacements, and primarily comes from foreign countries.

Carbon purity in graphite is a key consideration for both producers and customers. The higher the grade, the lower the milling and refining costs for producers, and for customers, higher grades offers more flexibility in product applications. There are three types of naturally occurring graphite: amorphous (or microcrystalline), flake (or 
crystalline), and vein/lump. Amorphous graphite is the lowest quality and most abundant. This form of graphite has very small crystal size that cannot be distinguished by the naked eye and is used for lower value graphite products and is the lowest priced graphite. Flake graphite is less common and of higher quality than amorphous graphite. Good quality flakes can be processed into expandable graphite (graphite that expands or exfoliates when heated) for other uses. Vein graphite is the highest quality and rarest graphite. It occurs in veins along intrusive contacts in solid lumps (Olson, 2012). Canada is a foremost producer of disseminated flake graphite deposits.

Only a small portion of carbon in the Earth's crust is in the form of graphite or diamond; the majority of carbon is contained in carbonate rocks, such as limestone, and organic matter, such as bituminous rock and fossil fuels. Graphite may be produced in any setting with favourable paleo-environment for the accumulation and preservation of organic materials (Simandl \& Kenan, 1997). The most common ways graphite is formed is by contact metamorphism of coal, which generally creates amorphous graphite, or from syngenetic metasediments which form disseminated flake graphite. Crystalline lump graphite is formed in epigenetic veins in high-grade metamorphic regions. Graphite also occurs as a trace mineral in ultrabasic rocks and pegmatites, but these are not economically viable (Krauss et al., 1998).

Disseminated flake graphite deposits are usually hosted in porphyroblastic and granoblastic marbles, paragneisses, and quartzites, with the most favorable host rocks being alumina-rich paragneisses and marbles in the upper amphibolite or granulite 
facies (Simandl \& Kenan, 1997). These geological environments are commonly found in the Canadian Precambrian shield (Mackinnon \& LeBaron, 1992) and most known deposits are of Precambrian age. The highest quality deposits are usually associated with rocks located at the contacts between marbles and paragneisses, and overall quality of graphite flake increase with intensity of regional metamorphism. Deposits are thickest within fold crests (Simandl \& Kenan, 1997). The typical depositional environment for disseminated flake graphite is composed of metasedimentary belts of granulite or upper amphibolite facies intruded by igneous rocks. Graphite deposits commonly form in clusters and are often stratiform lens-shaped or saddle-shaped. Economically viable deposits are several metres to tens of metres thick and hundreds of metres in strike length (Simandl \& Kenan, 1997).

The carbon atoms in graphite are arranged in parallel-stacked, planar honeycomblattice sheets. Another form of pure carbon is diamond, which has a 3D atomic structure where each atom is bound to four neighboring carbon atoms. Differences in atomic structure is what causes diamond to be very hard, a 10 on the Moh's hardness scale, and graphite to be very soft, a 1-2 on Moh's scale. It is also what causes graphite to have anisotropic conductivity; the conductivity along the sheet is higher than the conductivity crossing the lattice sheet. Because of this, the conductivity of graphite has a range of conductivity from $10 \mathrm{~S} / \mathrm{m}-0.1 \mathrm{~S} / \mathrm{m}$, or $0.1 \Omega \cdot \mathrm{m}-10 \Omega \cdot \mathrm{m}$. For EM systems, the best case scenario for graphite detection is a horizontal-oriented graphite deposit, where there is maximum coupling between the primary field and the graphite lattice sheets. Graphite is gray to black in colour, opaque, and usually has a metallic or greasy luster. It 
is flexible but not elastic, has a very high melting point of $3,927^{\circ} \mathrm{C}$, and a low density, ranging from $2.09-2.23 \mathrm{~g} / \mathrm{cm}^{3}$ and is the most electrically and thermally conductive of the non-metals. It is chemically inert and diamagnetic.

These properties are what make graphite an irreplaceable material in industrial applications and as a result, graphite is used in numerous applications such as refractories, steelmaking, brake linings, foundries, batteries, and lubricants. The next important development in the use of graphite will be in advanced automotive batteries and fuel cells. A single sheet of graphite is called graphene, which is very strong yet extremely lightweight, making it a very promising material for industrial use. However, technological developments are needed to mass-separate the graphene sheets from graphite before it is economically feasible.

Due to their high conductivity, graphite deposits are good exploration targets for EM methods, both airborne and ground. Induced polarisation (IP) methods are also used, however they are considered relatively expensive and in many cases too sensitive (Simandl \& Kenan, 1997). The classic geophysical signature of graphite deposits is a strong EM anomaly coincident with a weak magnetic anomaly. 


\section{Instrumentation}

\subsection{The IMAGEM system}

The IMAGEM system (Ricard, 2013, Figure 3.1) is a new ground-based timedomain electromagnetic (TDEM) system employing a unique and unorthodox configuration allowing for exceptional resolution whilst nulling the primary electromagnetic field. The system is under development by Devbrio Géophysique Inc. The system consists of an in-loop configuration (Figure 2.3b), where the transmitter loops around the dipole receiver which is located in the centre, both arranged in the horizontal coplanar configuration (Figure 3.4). Through magnetic flux modelling, the transmitter can be modelled as a $2.1 \mathrm{~m} \times 2.1 \mathrm{~m}$ square loop, which will be used to represent the IMAGEM system when modelling with AarhusInv.

The transmitter, composed of an AWG 3/0 (10.405 mm diameter) gauge aluminum wire, creates a half-sine pulse (Figure 3.2B), lasting $0.486 \mathrm{~ms}$ (the on-time), followed by $16.6 \mathrm{~ms}$ of off-time, which is then followed by the next pulse. The pulse alternates between positive and negative amplitude. One full cycle (one positive and one negative pulse) lasts $33.3 \mathrm{~ms}$ (Figure $3.2 \mathrm{~A}$ ); the system therefore operates at a base frequency of $30 \mathrm{~Hz}$. The receiver currently uses 110 channels: the first 25 record the ontime signal, and the following 85 record the off-time signal (Table 3.2). In the future, additional receivers could be added and positioned in different orientations.

The system carries a tablet computer at the front where the operator can see the current receiver readings, as well as other information such as battery life and GPS 
coordinates. The system can be operated on foot by two operators or towed by a vehicle. A real-time audio feedback system based on the measured response has also been designed for the IMAGEM system (Cunningham, 2013). A summary of the specifications of the IMAGEM system is presented in Table 3.1. 


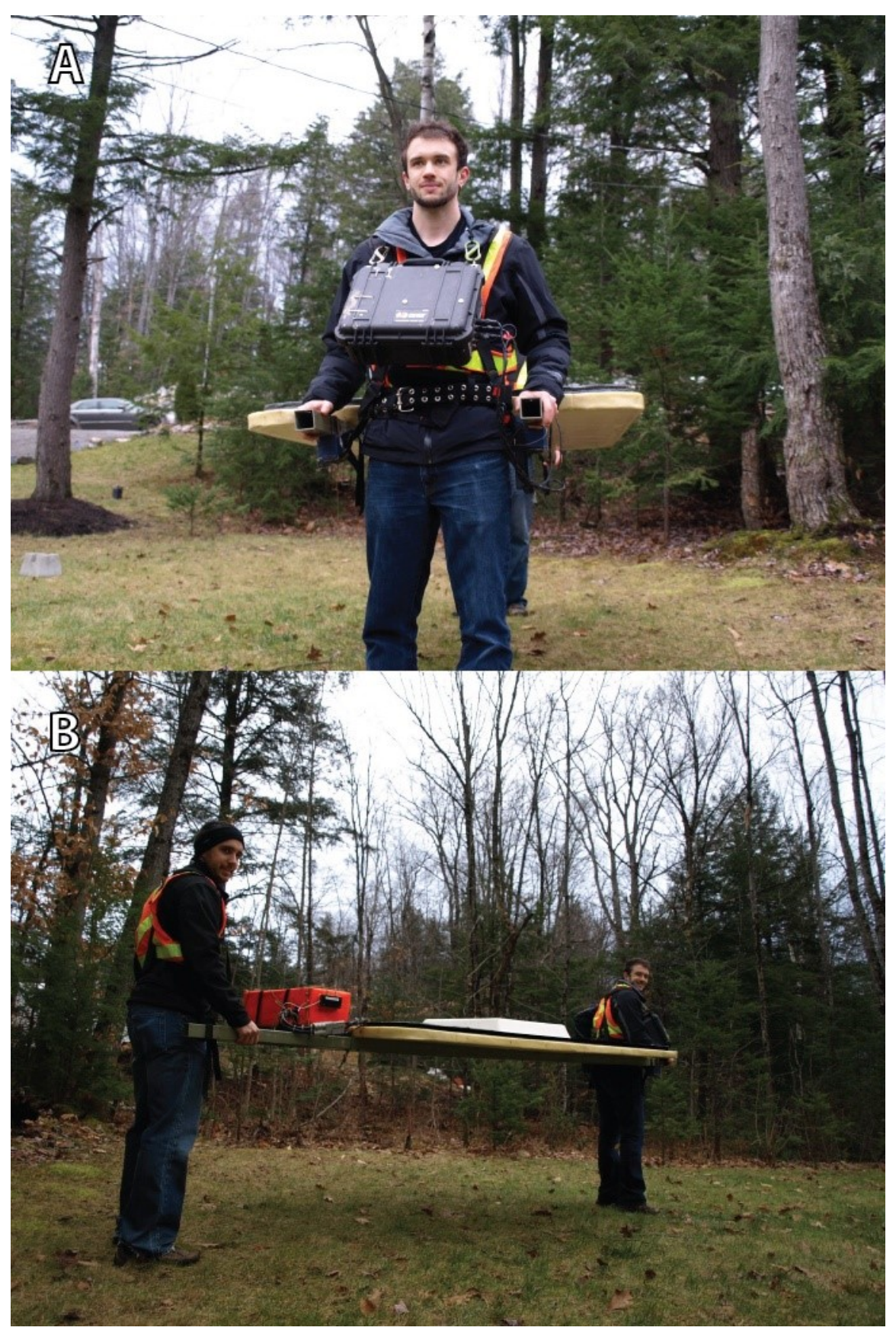

Figure 3.1. The IMAGEM system. [A] View of the front operator. The black box attached to the operator contains the tablet computer and other electronic hardware. The operator wears a harness that is attached to the frame of the system so the system can be carried hands-free. [B] View of the side of the system. The orange box contains the battery and other electronic hardware. The white box in the centre of the system covers the dipole receiver (Photos courtesy of Devbrio Géophysique Inc.). 
Table 3.1. Specifications of the IMAGEM system.

\begin{tabular}{|l|l|}
\hline \multirow{2}{*}{ Height above ground } & $0.8 \mathrm{~m}$ \\
\hline \multirow{2}{*}{ Length } & In-loop transmitter and receiver \\
\cline { 2 - 2 } Width & Horizontal co-planar geometry \\
\hline Transmitter & $3.7 \mathrm{~m}$ \\
\hline Type & $1.14 \mathrm{~m}$ \\
\hline Current & Horizontal square equivalent $\left(4.41 \mathrm{~m}^{2}\right.$ ) \\
\hline Number of turns & $700 \mathrm{~A}$ \\
\hline Moment & 1 \\
\hline Receiver & $\sim 3100 \mathrm{Am}^{2}$ \\
\hline Type & Horizontal Loop \\
\hline Radius & Variable \\
\hline Recording channels & $1-25$ (on-time) \\
\cline { 2 - 2 } & $26-110$ (off-time) \\
\hline Sampling frequency & $105 \mathrm{kS} / \mathrm{s}$ \\
\hline Signal & Alternating positive and negative half sine wave \\
\hline Waveform & $0.486 \mathrm{~ms}$ \\
\hline On-time duration & $16.180 \mathrm{~ms}$ \\
\hline Off-time duration & $30 \mathrm{~Hz}$ \\
\hline Sounding frequency &
\end{tabular}


(A)

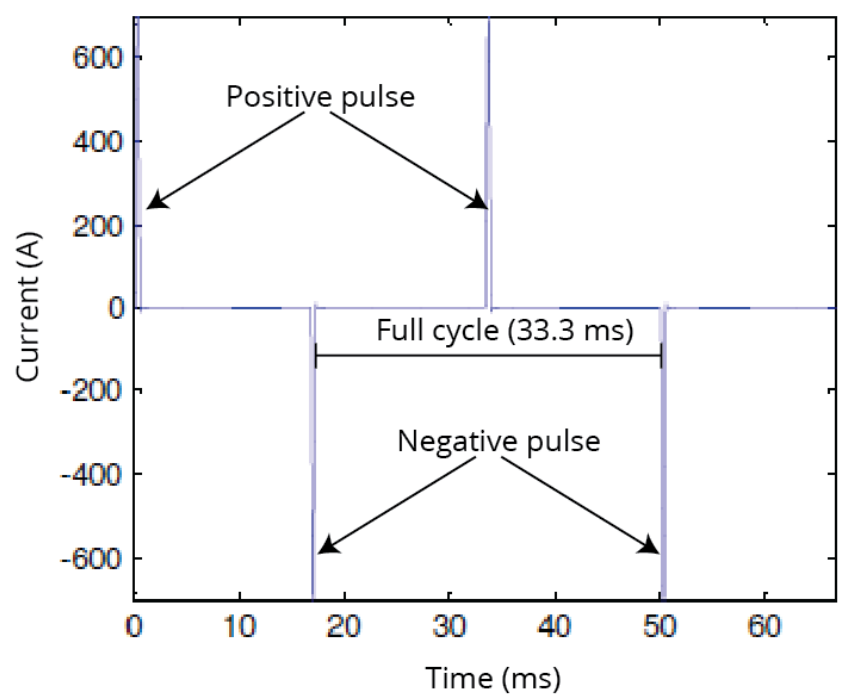

(B)

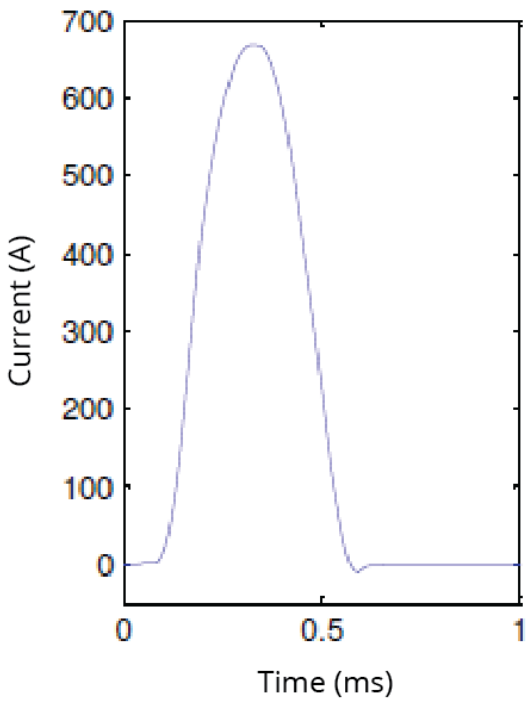

Figure 3.2. (A) Two full cycles of the IMAGEM transmitter. One cycle consists of a positive pulse and one negative pulse. (B) The half-sine pulse waveform used by the IMAGEM system (modified from Ricard, 2013). 
Table 3.2. Times after turn-off for the 110 channels of the IMAGEM system. 'Tx': transmitter.

\begin{tabular}{|c|c|c|c|c|c|c|c|c|}
\hline Channel & $\begin{array}{c}\text { Time after } \\
\text { turn-off (ms) }\end{array}$ & Tx & Channel & $\begin{array}{c}\text { Time after } \\
\text { turn-off (ms) }\end{array}$ & Tx & Channel & $\begin{array}{c}\text { Time after } \\
\text { turn-off (ms) }\end{array}$ & Tx \\
\hline 1 & N/A & ON & 38 & 0.124 & OFF & 75 & 1.580 & OFF \\
\hline 2 & N/A & ON & 39 & 0.133 & OFF & 76 & 1.690 & OFF \\
\hline 3 & N/A & ON & 40 & 0.143 & OFF & 77 & 1.800 & OFF \\
\hline 4 & N/A & ON & 41 & 0.152 & OFF & 78 & 1.920 & OFF \\
\hline 5 & N/A & ON & 42 & 0.162 & OFF & 79 & 2.060 & OFF \\
\hline 6 & N/A & ON & 43 & 0.181 & OFF & 80 & 2.200 & OFF \\
\hline 7 & N/A & ON & 44 & 0.190 & OFF & 81 & 2.350 & OFF \\
\hline 8 & N/A & ON & 45 & 0.210 & OFF & 82 & 2.510 & OFF \\
\hline 9 & N/A & ON & 46 & 0.220 & OFF & 83 & 2.690 & OFF \\
\hline 10 & $\mathrm{~N} / \mathrm{A}$ & ON & 47 & 0.240 & OFF & 84 & 2.870 & OFF \\
\hline 11 & N/A & ON & 48 & 0.260 & OFF & 85 & 3.070 & OFF \\
\hline 12 & N/A & ON & 49 & 0.280 & OFF & 86 & 3.280 & OFF \\
\hline 13 & N/A & ON & 50 & 0.300 & OFF & 87 & 3.500 & OFF \\
\hline 14 & N/A & ON & 51 & 0.310 & OFF & 88 & 3.740 & OFF \\
\hline 15 & $\mathrm{~N} / \mathrm{A}$ & ON & 52 & 0.330 & OFF & 89 & 3.990 & OFF \\
\hline 16 & $\mathrm{~N} / \mathrm{A}$ & ON & 53 & 0.360 & OFF & 90 & 4.270 & OFF \\
\hline 17 & $\mathrm{~N} / \mathrm{A}$ & ON & 54 & 0.380 & OFF & 91 & 4.560 & OFF \\
\hline 18 & N/A & ON & 55 & 0.410 & OFF & 92 & 4.870 & OFF \\
\hline 19 & N/A & ON & 56 & 0.440 & OFF & 93 & 5.200 & OFF \\
\hline 20 & N/A & ON & 57 & 0.480 & OFF & 94 & 5.560 & OFF \\
\hline 21 & $\mathrm{~N} / \mathrm{A}$ & ON & 58 & 0.500 & OFF & 95 & 5.940 & OFF \\
\hline 22 & N/A & ON & 59 & 0.540 & OFF & 96 & 6.340 & OFF \\
\hline 23 & N/A & ON & 60 & 0.580 & OFF & 97 & 6.780 & OFF \\
\hline 24 & N/A & ON & 61 & 0.620 & OFF & 98 & 7.240 & OFF \\
\hline 25 & 0.000 & RAMP & 62 & 0.660 & OFF & 99 & 7.730 & OFF \\
\hline 26 & 0.010 & OFF & 63 & 0.700 & OFF & 100 & 8.270 & OFF \\
\hline 27 & 0.019 & OFF & 64 & 0.760 & OFF & 101 & 8.830 & OFF \\
\hline 28 & 0.029 & OFF & 65 & 0.810 & OFF & 102 & 9.430 & OFF \\
\hline 29 & 0.038 & OFF & 66 & 0.870 & OFF & 103 & 10.080 & OFF \\
\hline 30 & 0.048 & OFF & 67 & 0.920 & OFF & 104 & 10.760 & OFF \\
\hline 31 & 0.057 & OFF & 68 & 0.990 & OFF & 105 & 11.500 & OFF \\
\hline 32 & 0.067 & OFF & 69 & 1.060 & OFF & 106 & 12.280 & OFF \\
\hline 33 & 0.076 & OFF & 70 & 1.130 & OFF & 107 & 13.110 & OFF \\
\hline 34 & 0.086 & OFF & 71 & 1.210 & OFF & 108 & 14.010 & OFF \\
\hline 35 & 0.095 & OFF & 72 & 1.300 & OFF & 109 & 14.960 & OFF \\
\hline 36 & 0.105 & OFF & 73 & 1.380 & OFF & 110 & 15.990 & OFF \\
\hline 37 & 0.114 & OFF & 74 & 1.480 & OFF & & & \\
\hline
\end{tabular}


The theoretical depth of penetration $d$ for the IMAGEM system can be calculated from the equation (Nabighian \& Macnae, 1991):

$$
d=\sqrt{\frac{2 t}{\sigma \mu}}
$$

where $t$ is the on-time duration, $\sigma$ is the conductivity of the ground, and $\mu$ is magnetic permeability, in this case it is equal to $\mu_{0}$ the permeability of free space. However, in practice, the depth of investigation is dependent on many factors, including the transmitter current, the geometry and configuration of the transmitter and receiver, the conductivity of the target and the level of noise, therefore the theoretical depth calculated is approximate. From equation (7, assuming an on-time duration of $0.486 \mathrm{~ms}$ (Table 3.1), a subsurface conductivity of $0.001 \mathrm{~s} / \mathrm{m}$, and no noise, the IMAGEM system has an approximate depth of penetration of $16 \mathrm{~m}$. 


\subsection{The MaxMin system}

The MaxMin system is a well-established ground-based EM system that has been used for high-resolution surveys since the 1970s. During the course of this research project, MaxMin data were acquired to compare them with the IMAGEM data.

The MaxMin I-8 (Maximum coupled-Minimum coupled, Apex Parametrics Limited n.d.) system is a frequency-domain electromagnetic (FDEM) slingram system (Figure 2.3C) made by Apex Parametrics Ltd of Markham, Ontario. It is composed of a transmitter and receiver (Figure 3.3) that are connected with a cable and are separated by a distance ranging from $25 \mathrm{~m}$ to $250 \mathrm{~m}$. It can use up to 5 transmitter frequencies $(222,444,888,1777,3555 \mathrm{~Hz})$, which have different depths of penetration. The receiver can also be oriented in different directions relative to the transmitter (Figure 3.4 ) to achieve horizontal coplanar (HCP), vertical coaxial (VCA), or perpendicular (PERP) configurations. The depth of penetration the MaxMin system depends on the frequency used, the conductivity of the subsurface, the coil separation, and the configuration. A general rule of thumb is to use the coil separation as an indicator of depth of penetration, usually between 0.75 to 1.5 times the coil separation, depending on the orientation (horizontal or vertical) of the target (Apex Parametrics Limited, n.d.). In the modelling presented in Chapter 5 and the case studies presented in Chapter 6, a coil separation of $50 \mathrm{~m}$ is used, resulting in a depth of penetration of approximately $37.5 \mathrm{~m}$ to $75 \mathrm{~m}$. The MaxMin system is equipped with a portable computer that is interfaced with the receiver and is used for digital data processing, display, storage, and transfer. 
The MaxMin I-8 transmitter consists of an air-core loop that is battery powered and carried by one person. The receivers are ferromagnetic-cored coils mounted on either side of the receiver console with their axes vertical when used in the horizontal coplanar (HCP, Figure 3.4) configuration. The cable connecting the transmitter and receiver provides a reference signal which allows for the compensation of the primary field by subtracting it from the resulting EM response, and also serves as a phase reference for synchronous detection of the secondary field. A switched-capacitor bandpass filter and noise clipping circuits before the synchronous detectors provide narrow system bandwidth and relative immunity to sferics (Frischknecht et al., 1991). The HCP configuration is normally used, but the receiver can be positioned horizontally for perpendicular (PERP, Figure 3.4) orientation. Vertical coplanar measurements (VCP, Figure 3.4) can also be made, but with more difficulty.

The MaxMin I-8 has the following principal features:

- Its portable computer displays and stores the in-phase and quadrature readings, their standard deviations, corresponding apparent ground conductivity values, and line station number.

- It uses advanced electronic (active and digital) filtering to reduce the interference effects of power lines and atmospheric noise.

- It has warning lights to indicate invalid readings.

- It uses a built-in tilt meter and computed coil orientation and separation information to ease rough terrain surveys. 


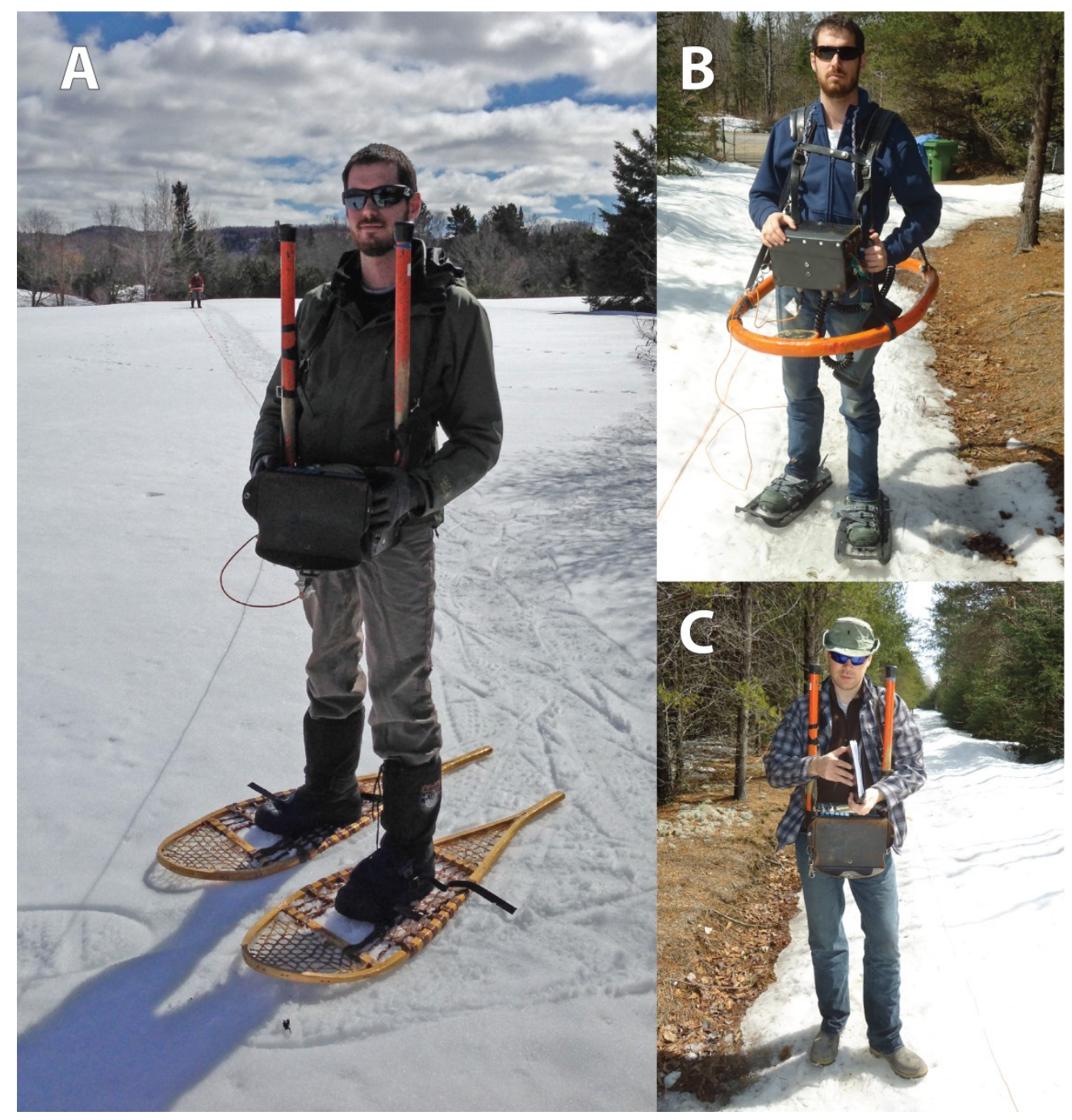

Figure 3.3. The MaxMin I-8 system. [A] The receiver (foreground) and transmitter (background) are connected by a cable. [B] The transmitter loop. [C] The receiver coils, positioned vertically for the HCP configuration (photos courtesy of Devbrio Géophysique Inc.). 

1. $\stackrel{T}{=}$
R HCP
2.
』 PERP
3.
(1) $v C p$
4. \
』 vCA

Figure 3.4. Common transmitter-receiver configurations for FDEM systems. HCP=Horizontal coplanar. PERP=Perpendicular. VCP=Vertical coplanar. VCA=Vertical coaxial. The MaxMin system most commonly uses the HCP configuration, but can also employ the PERP and VCP configurations (Nabighian \& Macnae, 1991). 


\subsection{Comparing the IMAGEM system and the MaxMin I-8 system}

The MaxMin system and the IMAGEM system differ in many ways. The most fundamental difference is that the MaxMin system is a frequency-domain system, whereas the IMAGEM system is a time-domain system. The MaxMin system uses a slingram configuration (Figure 2.3C); meaning the transmitter and receiver are separated by a fixed distance. The IMAGEM system uses an in-loop configuration (Figure 2.3B), thus the receiver is located in the centre of the transmitter. Therefore, the manner in which both systems attenuate the effect of primary field is different: the MaxMin system places the receiver far from the transmitter; the IMAGEM system creates a null field where the receiver is located.

The two systems record data in different ways. The MaxMin system measures at fixed, pre-determined 'stations'. The IMAGEM system is continuously recording as the two operators are walking along traverses; no stations are needed. This allows for a better spatial resolution because more measurements are made at very short intervals.

The MaxMin system is able to use different frequencies, which affect the induction number, and therefore the conductor response, and the system can vary the distance between the transmitter and receiver, which affects the footprint of the system and also the depth of penetration. The IMAGEM system cannot change its footprint or its depth of penetration. The MaxMin system has a depth of penetration larger than the IMAGEM system of $37.5 \mathrm{~m}$ to $75 \mathrm{~m}$ (Section 3.2) compared to an estimated $20 \mathrm{~m}$ (Section 3.1). 
From a logistical standpoint, the IMAGEM system has the advantage of being able to measure continuously, allowing for faster surveys. The MaxMin system has a cable connecting the transmitter and the receiver, which is cumbersome, especially in forested environments. Both systems require two persons to operate. 


\section{AarhusInv software}

AarhusInv (Auken et al., 2014, formerly named 'em1dinv') is a software program for the forward modelling and inversion of electrical and electromagnetic data for geophysical investigations. The software, developed at Aarhus University, Denmark, supports transient electromagnetic, frequency-domain electromagnetic, helicopterborne frequency-domain, direct current, magnetic resonance sounding, magnetotelluric, and surface wave dispersion systems. The base code is written in Fortran 95/77. The program itself is a command line application and therefore lacks any graphical user interface. All input and output are provided using ASCII files (Figure 4.1Figure 4.3). The software has great flexibility in the way it handles system configurations, allowing almost any transmitter and receiver shape and size, which is necessary to model the IMAGEM system.

There are two files used by the program: the MOD file (Figure 4.1), which contains the information about the model itself, and the TEM file (Figure 4.2) which contains the data and information about the system. When an inversion or forward model is performed, an EMO file is output (Figure 4.3). Once the input files are ready, they are passed on to the general inversion module which initiates the iterative inversion process. The program then begins the process of minimizing the residuals, which are the differences between the observed responses and the corresponding predicted computed responses. During this process, the inversion module calls the forward response module for forward response updates and derivatives. Once the inversion is complete, the result is sent to the general model analysis module, which performs a 
model parameter sensitivity analysis. The depth of investigation is then calculated based on the final inverted model. Following this calculation, a result file is written in ASCII format (Figure 4.3, Auken et al., 2014).

Because of the IMAGEM system's unconventional transmitter design, the exact shape of the transmitter cannot be modelled. However, results of magnetic flux simulations have shown that, at depths greater than approximately $2 \mathrm{~m}$, the IMAGEM transmitter is equivalent to a square loop measuring $2.1 \mathrm{~m} \times 2.1 \mathrm{~m}$. Therefore, the IMAGEM will be modelled as such in AarhusInv. The IMAGEM's relatively small receiver will be modelled as a point-source B-field (magnetic field) receiver measuring in the Zdirection, located in the centre of the transmitter loop (Figure 4.4). 


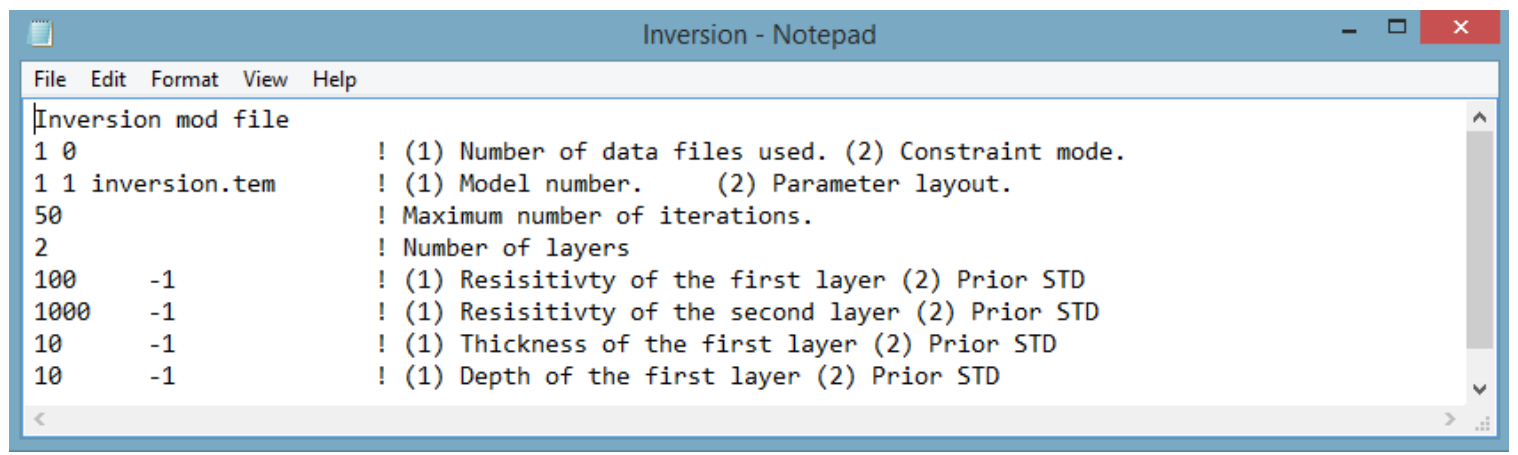

Figure 4.1. The MOD file contains the information about the initial guess model and some inversion parameters. A constraint mode of 0 means no constraint is applied. Inversion.tem is the TEM file the program will use. Prior STD is the factor within which the parameters may vary. The value of -1 means the parameters are free to vary. 


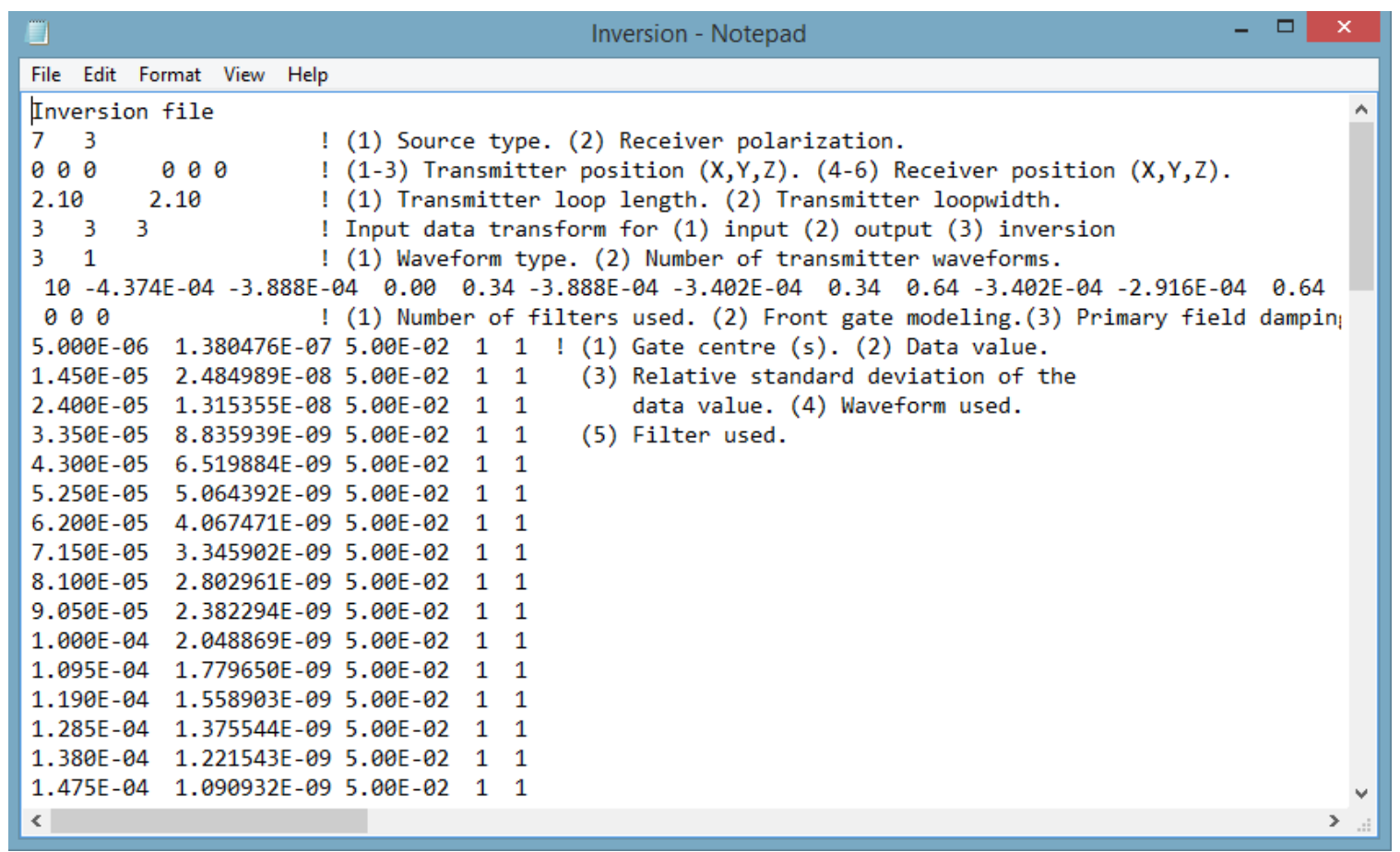

Figure 4.2. The TEM file contains the information about the system and the data to invert. In this example, the ' 7 ' on line 2 represents a rectangular loop transmitter and the ' 3 ' a Z-direction magnetic field receiver. The values on line 3 represent the position of the transmitter and receiver. In this case, both are coincident and centered (in-loop configuration). The three values of ' 3 ' on line 5 means the data is input, transformed, and output as $\mathrm{dB} / \mathrm{dt}\left(\mathrm{V} / \mathrm{m}^{2}\right)$. Line 6 states the waveform is user-defined; it is then defined in line 7. Finally, starting from line 9 and on, the data is input, starting with the channel centre time on the left, followed by the value of the response at that channel, then the standard deviation of the data value, then which waveform is used and which low pass filter is used. In this case, no filter is used. 


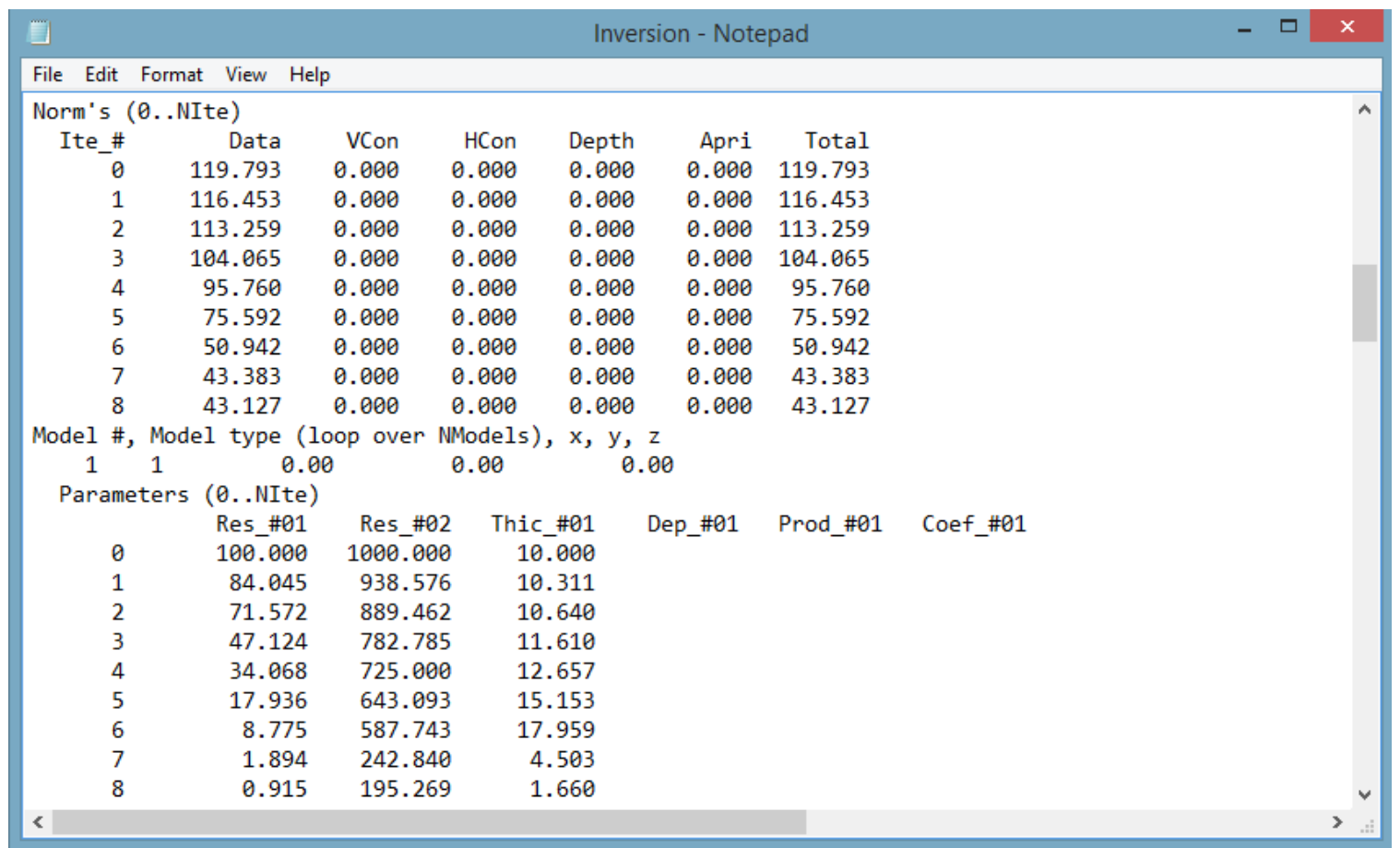

Figure 4.3. The EMO file, which is the output file after an inversion or forward model. In this example, the results of an inversion are presented. The bottom half of the figure, under 'Parameters', shows the resulting inverted model, including values of resistivity and thickness for every iteration from the initial guess model to the final inverted model. 


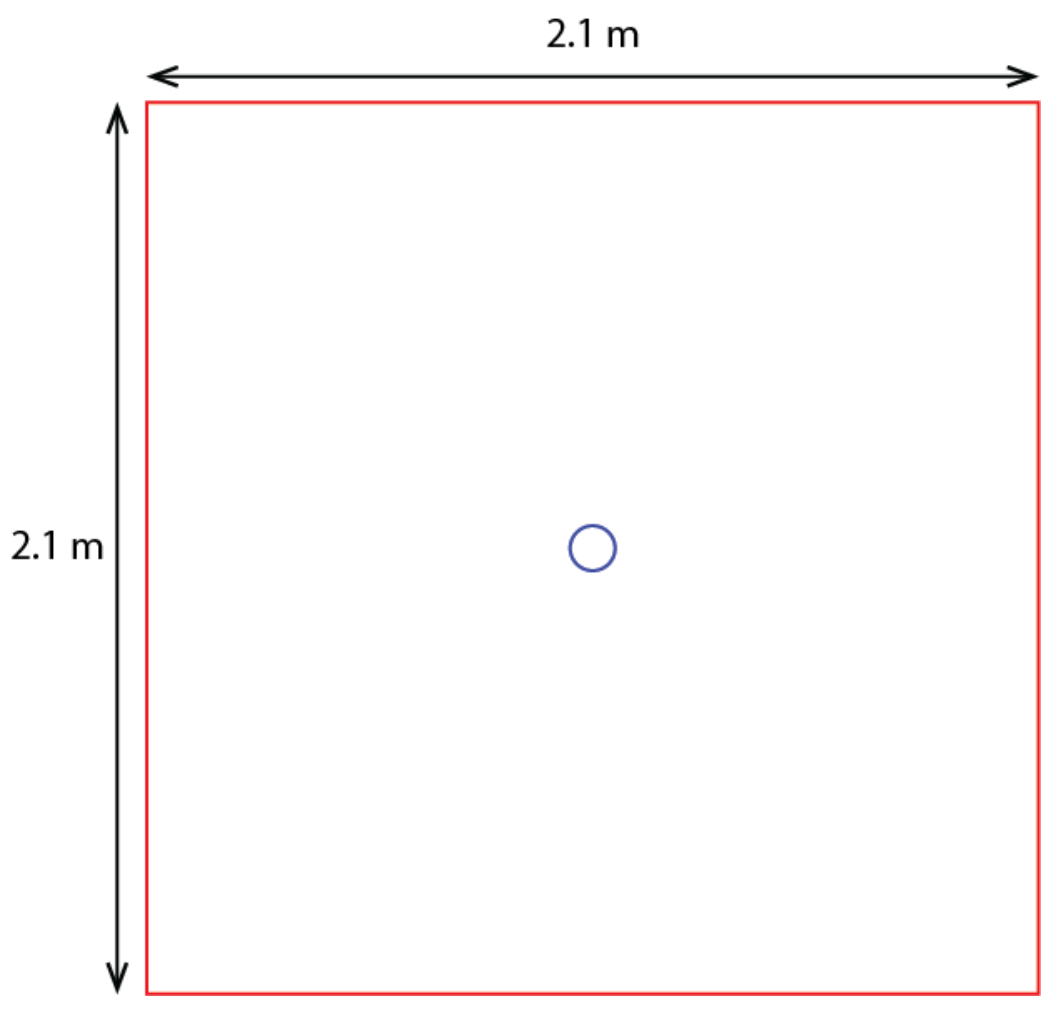

Figure 4.4. Representation of the IMAGEM's configuration used for modelling with AarhusInv. The red square represents the transmitter loop. The blue circle represents the receiver dipole. 


\section{Forward modelling and inversion of synthetic data}

\subsection{Forward modelling}

To gain insight into the responses of the IMAGEM system for different geological scenarios, and to test the capabilities and compatibility of AarhusInv with the IMAGEM system, forward modelling and the subsequent inversion of these forward models were performed. A forward model is, in essence, a calculation of the response expected for given model parameters. It is the opposite of an inversion, where the values of the model parameters must be determined based on the observed data.

The forward modelling and inversion process used with synthetic data is illustrated in Figure 5.1. A user generated initial 'true' model, representing a geological model, is processed using forward modelling to generate a decay curve. Synthetic noise is then added to the decay curve, and this noisy decay curve is inverted to generate a geological model, called the inverted model. The initial model and inverted model are then compared to each other.

A summary of the model parameters used for forward modelling and inversion is presented in Table 5.1. The first and simplest model used is that of a half-space with varying resistivity (Figure 5.2). Next are the 'overburden resistivity' models, which consist of a conductive overburden layer overlaying a resistive half-space and where only the resistivity of the overburden layer is varied (Figure 5.3). The last models are the 'overburden thickness' models, which also consist of a conductive overburden layer overlaying a resistive half-space, but only the thickness of the overburden layer is varied 
(Figure 5.4). These models are used to represent the typical geology of the Canadian

Precambrian shield, which usually consist of conductive overburden layers, composed of glacial till deposited during the last ice age, overlaying a resistive basement composed of metamorphic rock. 


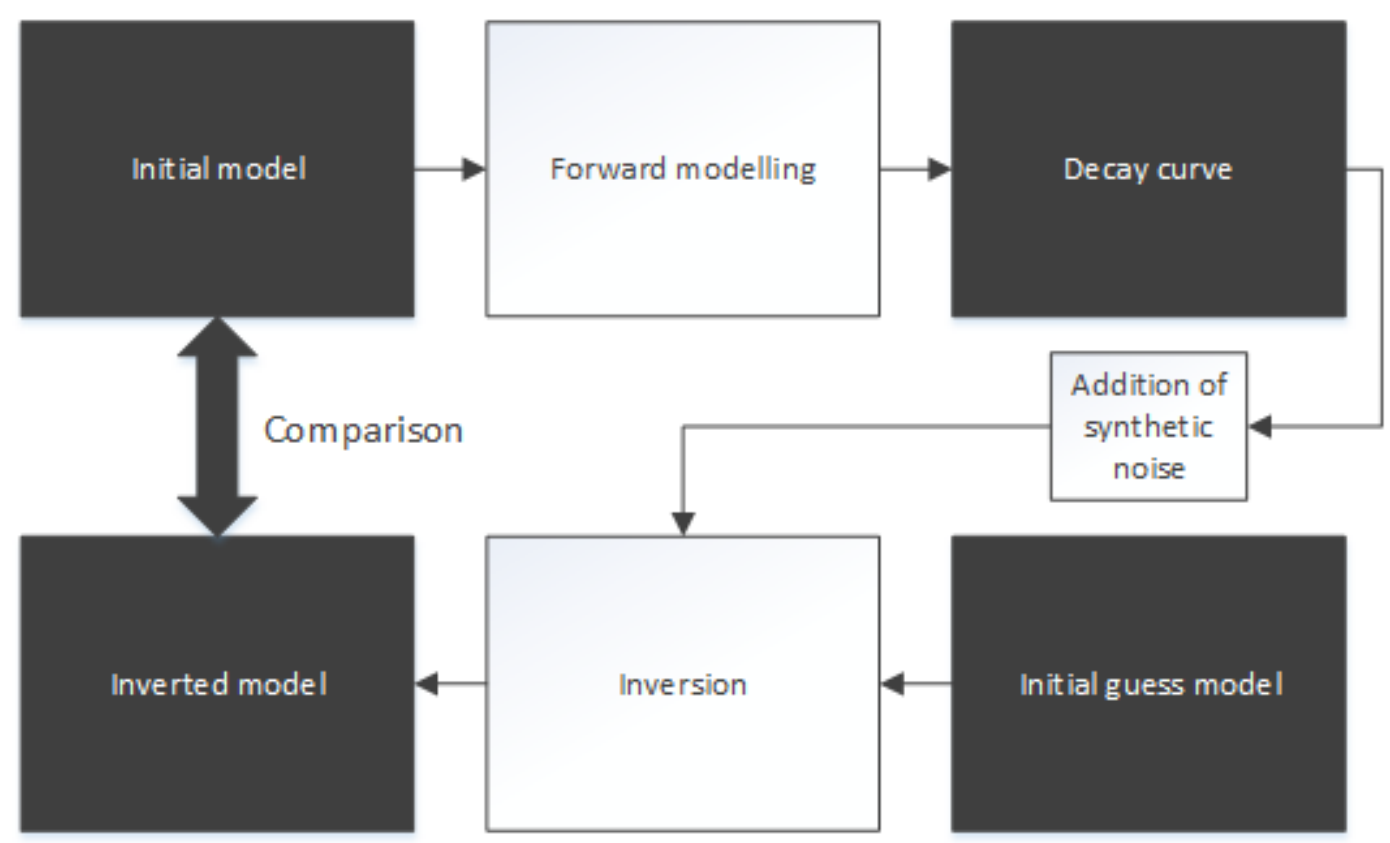

Figure 5.1. The forward modelling and inversion process for synthetic data. The black boxes represent data products, and white boxes represent operations. 
Table 5.1. Summary of the model parameters used for forward modelling and inversion using AarhusInv.

\begin{tabular}{|c|c|c|c|c|}
\hline \multirow{2}{*}{\multicolumn{2}{|c|}{ Models }} & \multicolumn{2}{|c|}{ Resistivity $(\Omega \cdot m)$} & Thickness (m) \\
\hline & & Layer 1 & Layer 2 & Laver 1 \\
\hline \multirow{2}{*}{ Half-space models } & Half-space $[A]$ & 1 & N/A & $\infty$ \\
\hline & Half-space [B] & 100 & $\mathrm{~N} / \mathrm{A}$ & $\infty$ \\
\hline \multirow{2}{*}{$\begin{array}{l}\text { Overburden resistivity } \\
\text { models }\end{array}$} & Overburden resistivity $[A]$ & 1 & 1000 & 10 \\
\hline & Overburden resistivity $[B]$ & 100 & 1000 & 10 \\
\hline \multirow{2}{*}{$\begin{array}{l}\text { Overburden thickness } \\
\text { models }\end{array}$} & Overburden thickness [A] & 10 & 1000 & 5 \\
\hline & Overburden thickness [B] & 10 & 1000 & 20 \\
\hline
\end{tabular}




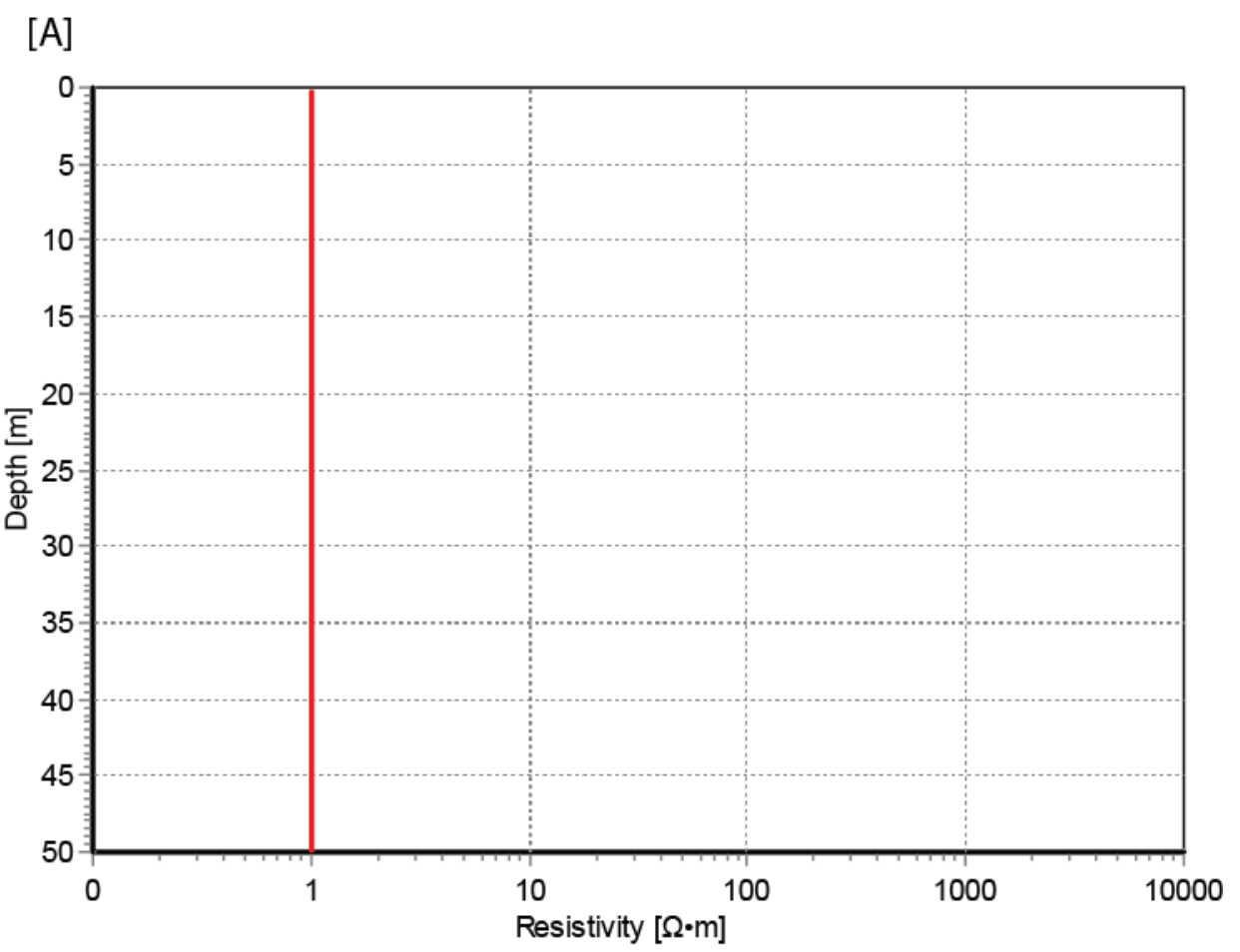

$[\mathrm{B}]$

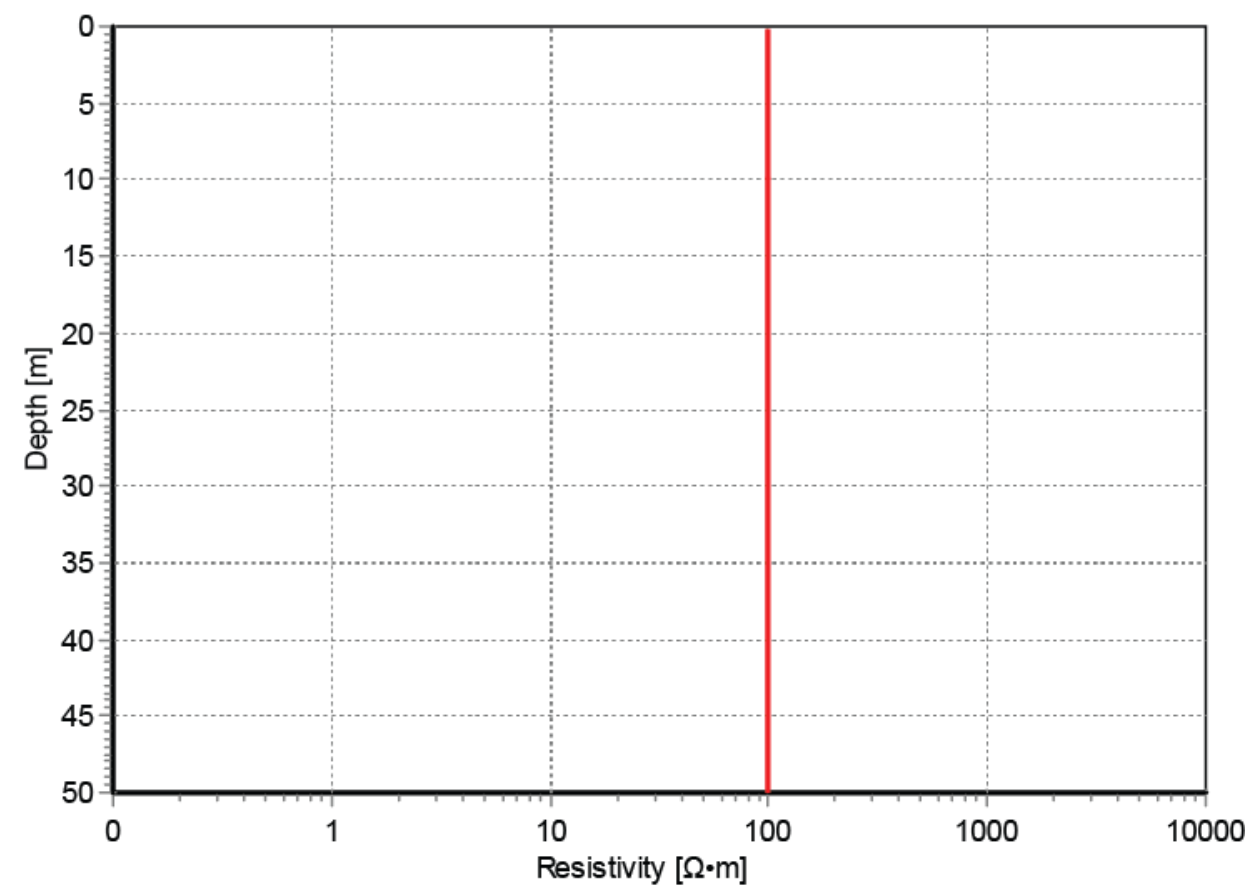

Figure 5.2. Visual representation of the half-space models. [A] $1 \Omega \cdot m$ half-space. [B] $100 \Omega \cdot m$ half-space. 


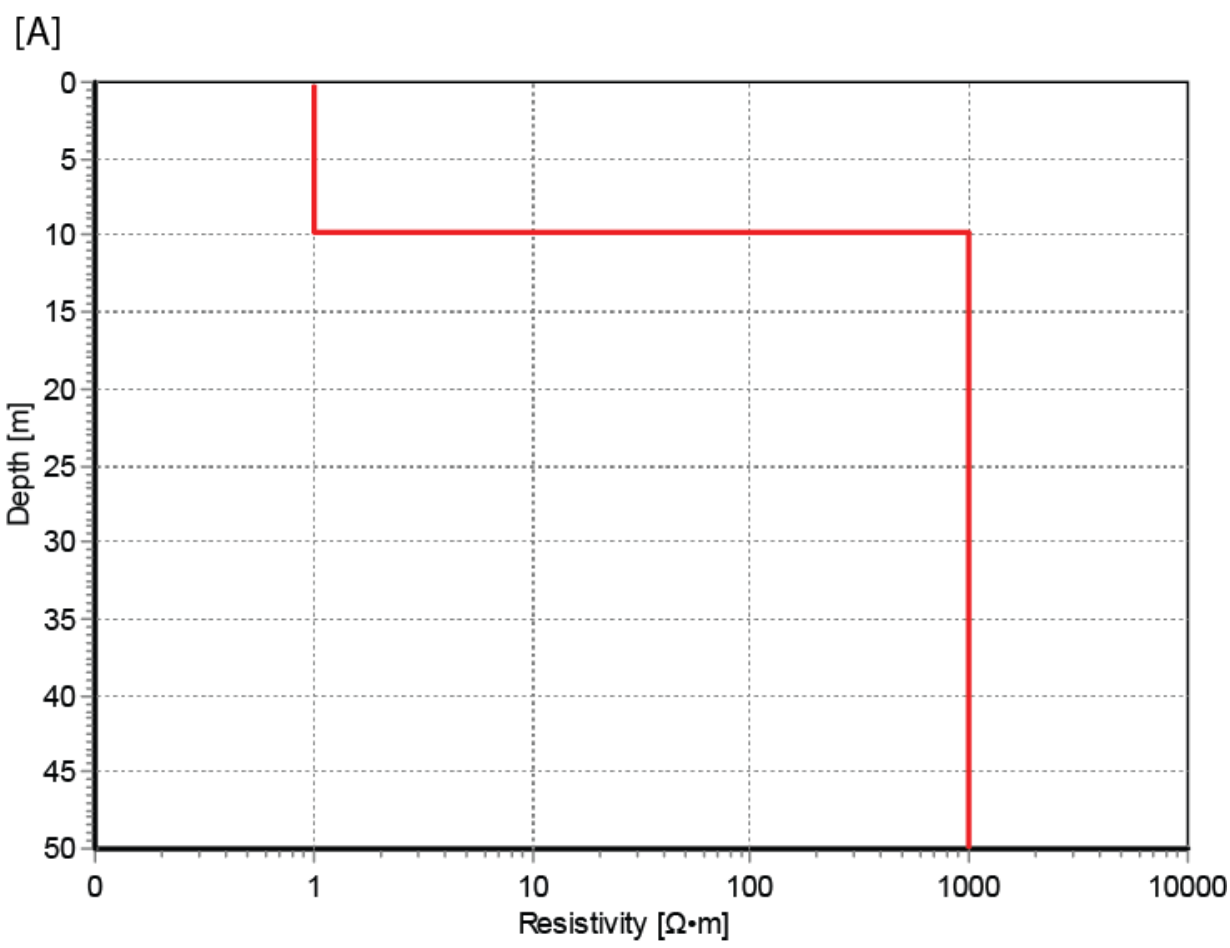

[B]

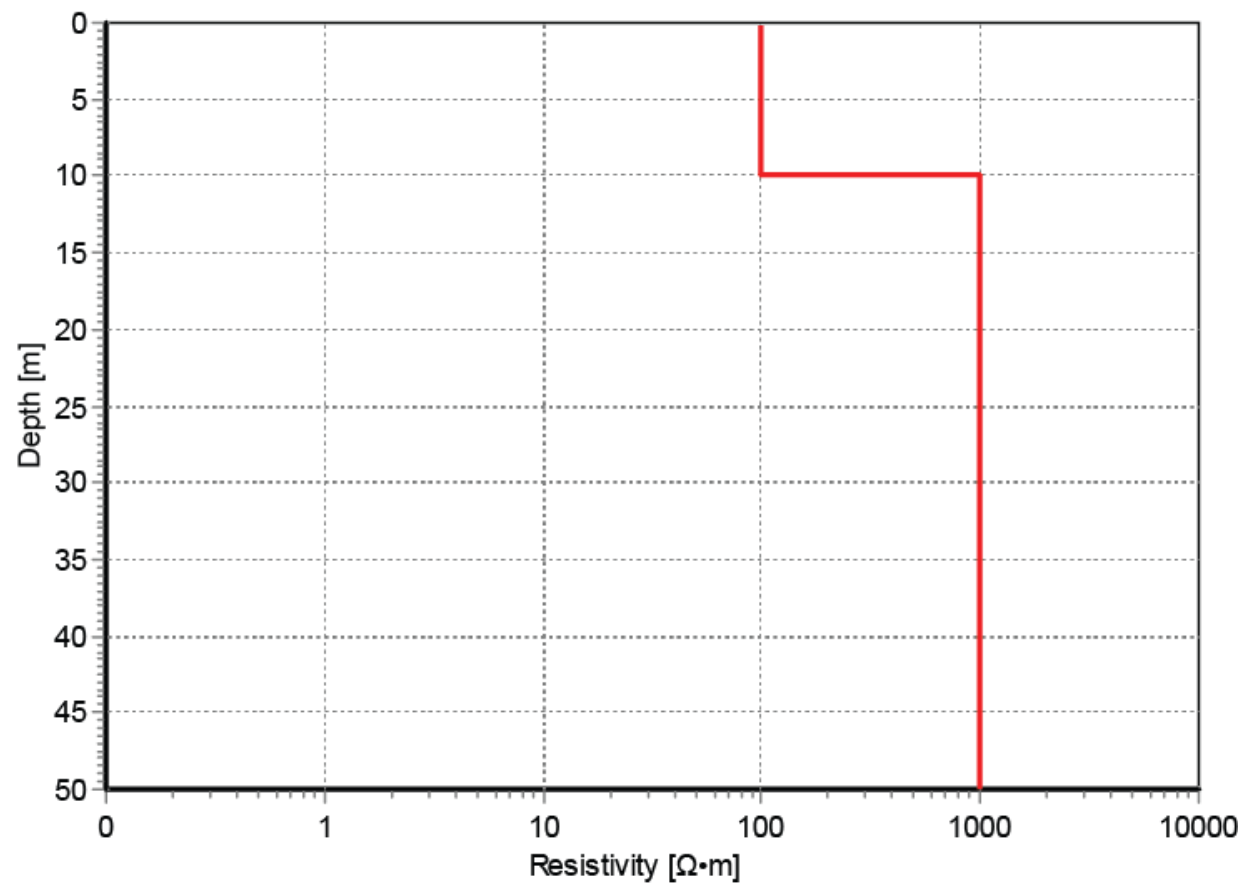

Figure 5.3. Visual representation of the overburden resistivity models, where only the resistivity of the overburden layer is varied. [A] consists of a $10 \mathrm{~m}$ thick overburden layer with a resistivity of $1 \Omega \cdot \mathrm{m}$ overlying a $1000 \Omega \cdot \mathrm{m}$ half-space. [B] consists of a $10 \mathrm{~m}$ thick overburden layer with a resistivity of 100 $\Omega \cdot m$ overlying a $1000 \Omega \cdot m$ half-space. 


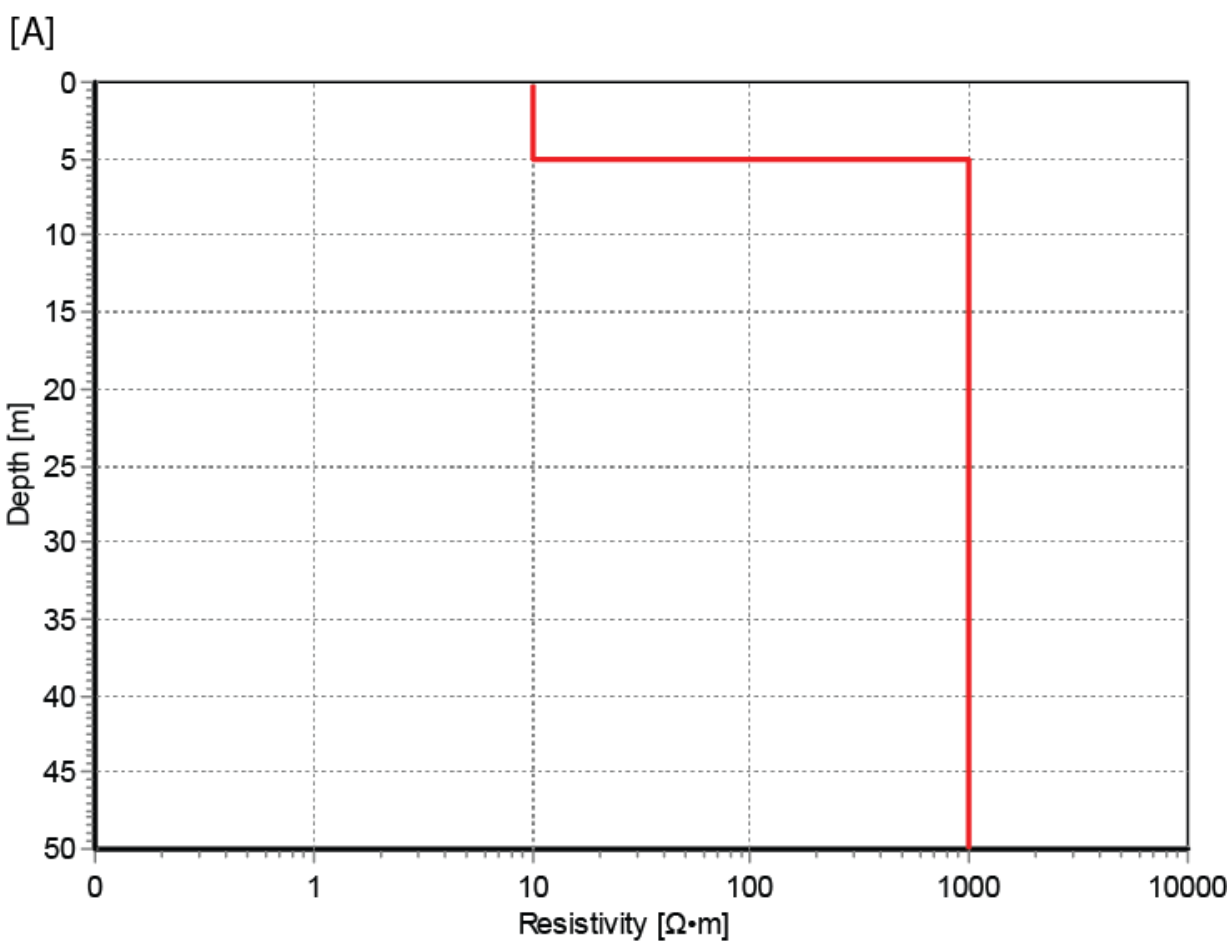

$[\mathrm{B}]$

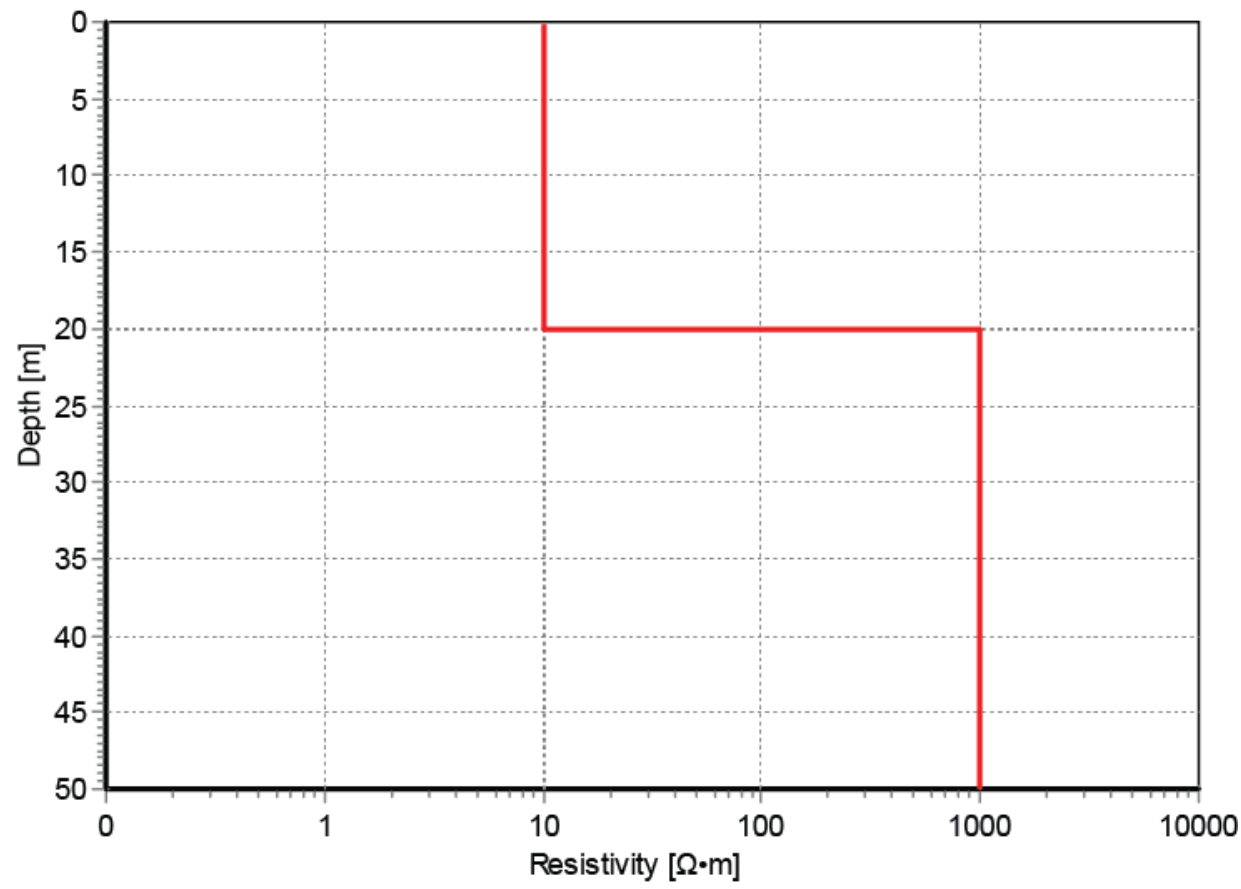

Figure 5.4. Visual representation of the overburden thickness models, where only the thickness of the overburden layer is varied. [A] consists of a $5 \mathrm{~m}$ thick overburden layer with a resistivity of $10 \Omega \cdot \mathrm{m}$ overlying a $1000 \Omega \cdot \mathrm{m}$ half-space. [B] consists of a $20 \mathrm{~m}$ thick overburden layer with a resistivity of $10 \Omega \cdot \mathrm{m}$ overlying a $1000 \Omega \cdot \mathrm{m}$ half-space. 


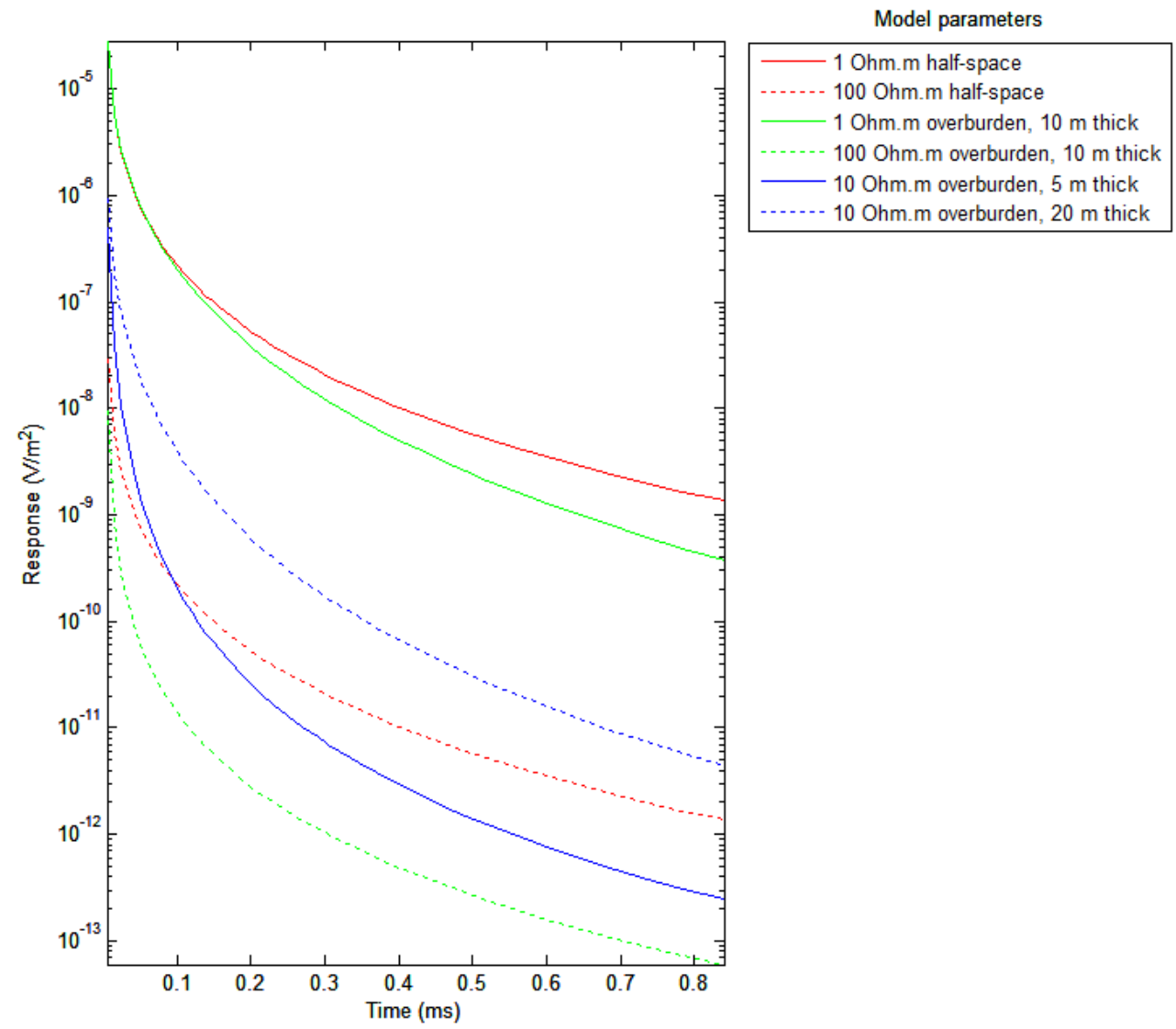

Figure 5.5. Synthetic decay curves (no noise added) generated by forward modelling the half-space models (red), overburden resistivity models (green), and overburden thickness models (blue). 
The result of the forward modelling is displayed in Figure 5.5. Looking at the halfspace models, the higher resistivity causes a lower response (compare the solid red and dotted red curves). The two curves, however, have the same shape. For the overburden resistivity models, the higher resistivity model (dotted blue curve) has a lower response and the lower resistivity model (solid blue curve) has a higher response. The two curves have the same shape. In the overburden thickness models, the thicker overburden layer (dotted green curve) has a higher response, and the thinner overburden layer (solid green curve) has a smaller response. The two curves do not have the same shape.

The highest response at time $t=0 \mathrm{~ms}$ is achieved by the $1 \Omega \cdot \mathrm{m}$ half-space model (solid red line) and the $1 \Omega \cdot \mathrm{m}$ overburden model (solid blue line). This is expected since they are the two models where the conductivity of the near-subsurface is highest. The decay curves of these two models deviate with time. This is due to the resistive halfspace underlying the conductive overburden layer, which causes the curve to decay faster relative to that of the half-space model. The next highest response at time $t=0 \mathrm{~ms}$ corresponds to the $10 \Omega \cdot \mathrm{m}$ and $20 \mathrm{~m}$ thick overburden layer (dotted green line). This model has a larger response than the $10 \Omega \cdot \mathrm{m}$ and $5 \mathrm{~m}$ thick overburden model (solid green line). Although both models contain a resistive half-space, the $20 \mathrm{~m}$ thick model has much more conductive material before reaching the half-space, causing the response to be stronger. The decay curve of the $100 \Omega \cdot \mathrm{m}$ half-space model crosses the decay curve of the $10 \Omega \cdot \mathrm{m}$ and $5 \mathrm{~m}$ thick overburden model. This is because, near the surface, the overburden model is more conductive. But below the $5 \mathrm{~m}$ thick overburden 
layer in the overburden model, the $1,000 \Omega \cdot \mathrm{m}$ underlying half-space is much more resistive than the $100 \Omega \cdot m$ half-space of the half-space model.

In general, the resistivity of the surface/near-subsurface material controls the amplitude at $t=0 \mathrm{~ms}$. The presence of material of increasing resistivity with depth controls the shape of the decay curve over time. As the subsurface becomes more resistive, the curve decays more rapidly.

To help with better understanding survey results of the IMAGEM system, forward modelling of a typical geological situation consisting of a graphite-rich layer underlying a conductive overburden layer and overlaying a resistive basement is performed. The depth and thickness of the graphite layer is varied to see the effects on the response of the IMAGEM system. A summary of the depths and thicknesses is presented in Table 5.2. The resistivity of the three layers is presented in Table 5.3. 
Table 5.2. Summary of the different models analyzed with the depth and thickness of the graphite layer.

\begin{tabular}{|c|c|c|}
\hline Model & Depth to graphite layer (m) & Thickness of graphite layer (m) \\
\hline 1 & 1 & 1 \\
\hline 2 & 1 & 5 \\
\hline 3 & 1 & 10 \\
\hline 4 & 1 & 15 \\
\hline 5 & 1 & 20 \\
\hline 6 & 5 & 1 \\
\hline 7 & 5 & 5 \\
\hline 8 & 5 & 10 \\
\hline 9 & 5 & 15 \\
\hline 10 & 5 & 20 \\
\hline 11 & 10 & 1 \\
\hline 12 & 10 & 5 \\
\hline 13 & 10 & 10 \\
\hline 14 & 10 & 15 \\
\hline 15 & 10 & 20 \\
\hline 16 & 15 & 1 \\
\hline 17 & 15 & 5 \\
\hline 18 & 15 & 10 \\
\hline 19 & 15 & 15 \\
\hline 20 & 15 & 20 \\
\hline 21 & 20 & 1 \\
\hline 22 & 20 & 5 \\
\hline 23 & 20 & 10 \\
\hline 24 & 20 & 15 \\
\hline 25 & 20 & 20 \\
\hline
\end{tabular}


Table 5.3. Resistivity values for the three layers of the graphite models. Layer 2 represents the graphite layer.

\begin{tabular}{|c|c|c|c|}
\hline Model & Resistivity of layer $\mathbf{1}(\mathbf{\Omega} \cdot \mathbf{m})$ & Resistivity of layer $\mathbf{2}(\mathbf{\Omega} \cdot \mathbf{m})$ & Resistivity of layer $\mathbf{3}(\mathbf{\Omega} \cdot \mathbf{m})$ \\
\hline A & 100 & 0.1 & 1000 \\
\hline B & 100 & 1.0 & 1000 \\
\hline C & 100 & 10 & 1000 \\
\hline
\end{tabular}




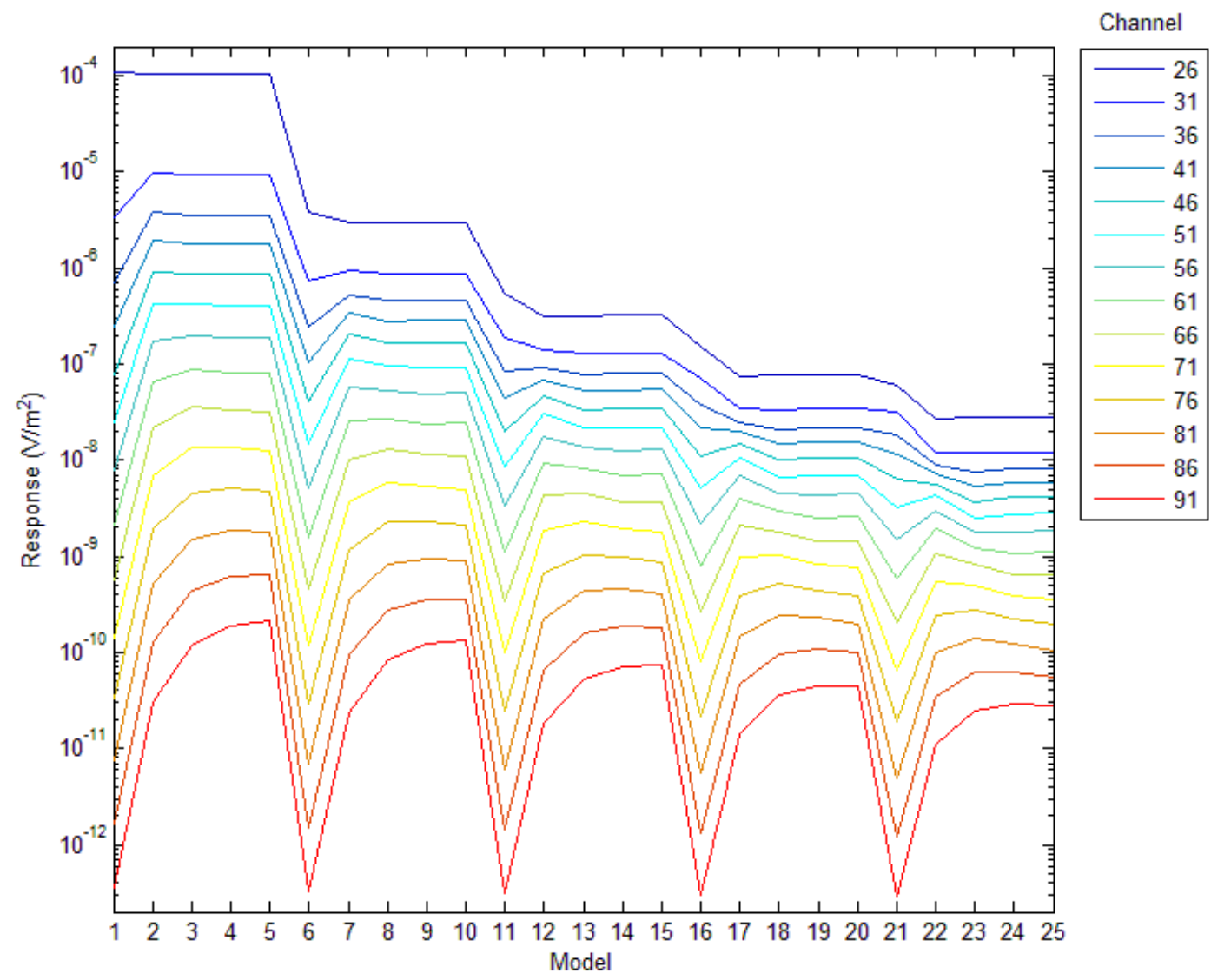

Figure 5.6. Response of multiple channels for graphite model A. Every 5 models shares the same graphite layer depth, but with increasing graphite layer thickness. A summary of the depth and thickness of the graphite layer represented on the abscissa is presented in Table 5.2. The model resistivity used is presented in Table 5.3. 


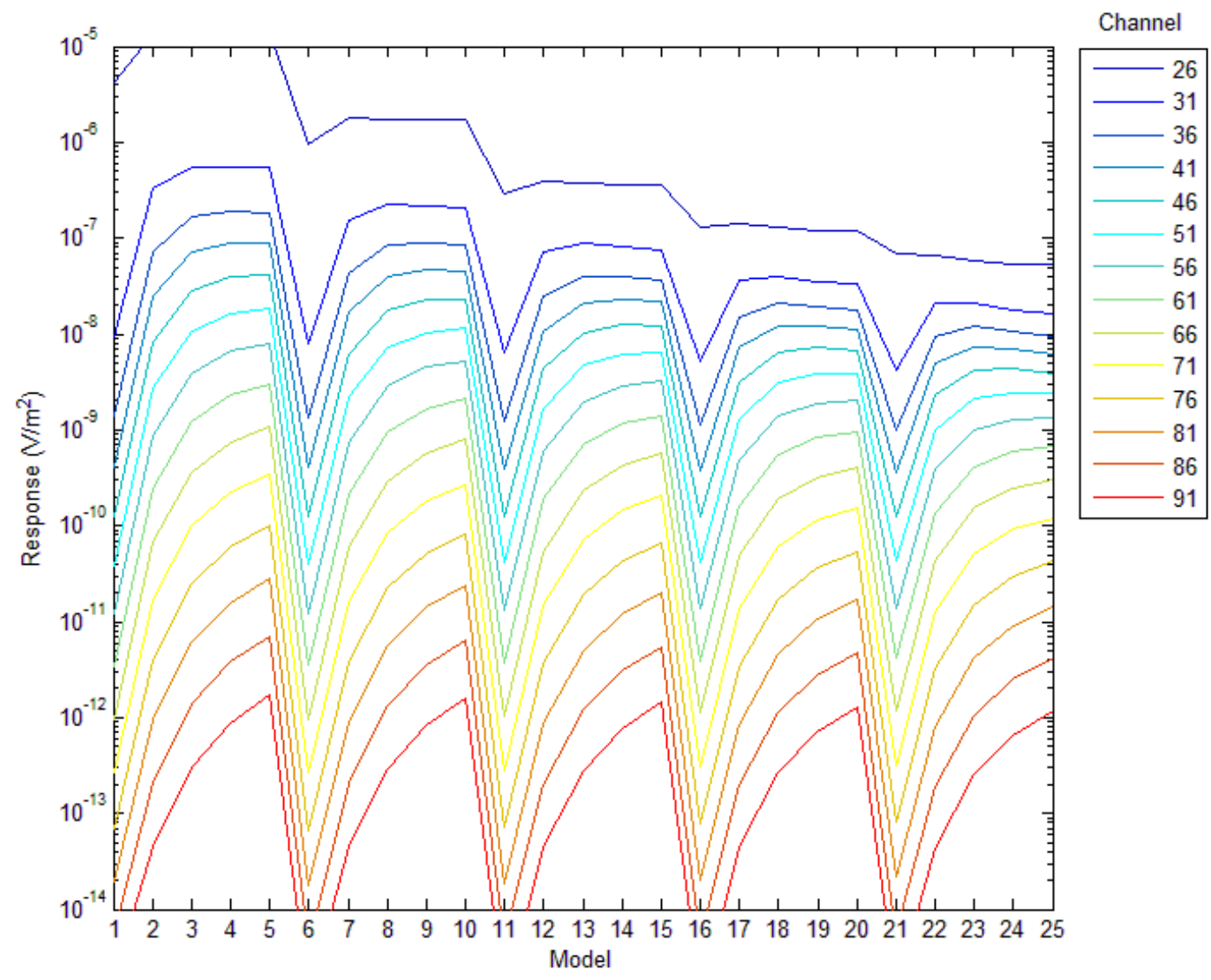

Figure 5.7. Response of multiple channels for graphite model B. Every 5 models shares the same graphite layer depth, but with increasing graphite layer thickness. A summary of the depth and thickness of the graphite layer represented on the abscissa is presented in Table 5.2. The model resistivity used is presented in Table 5.3. 


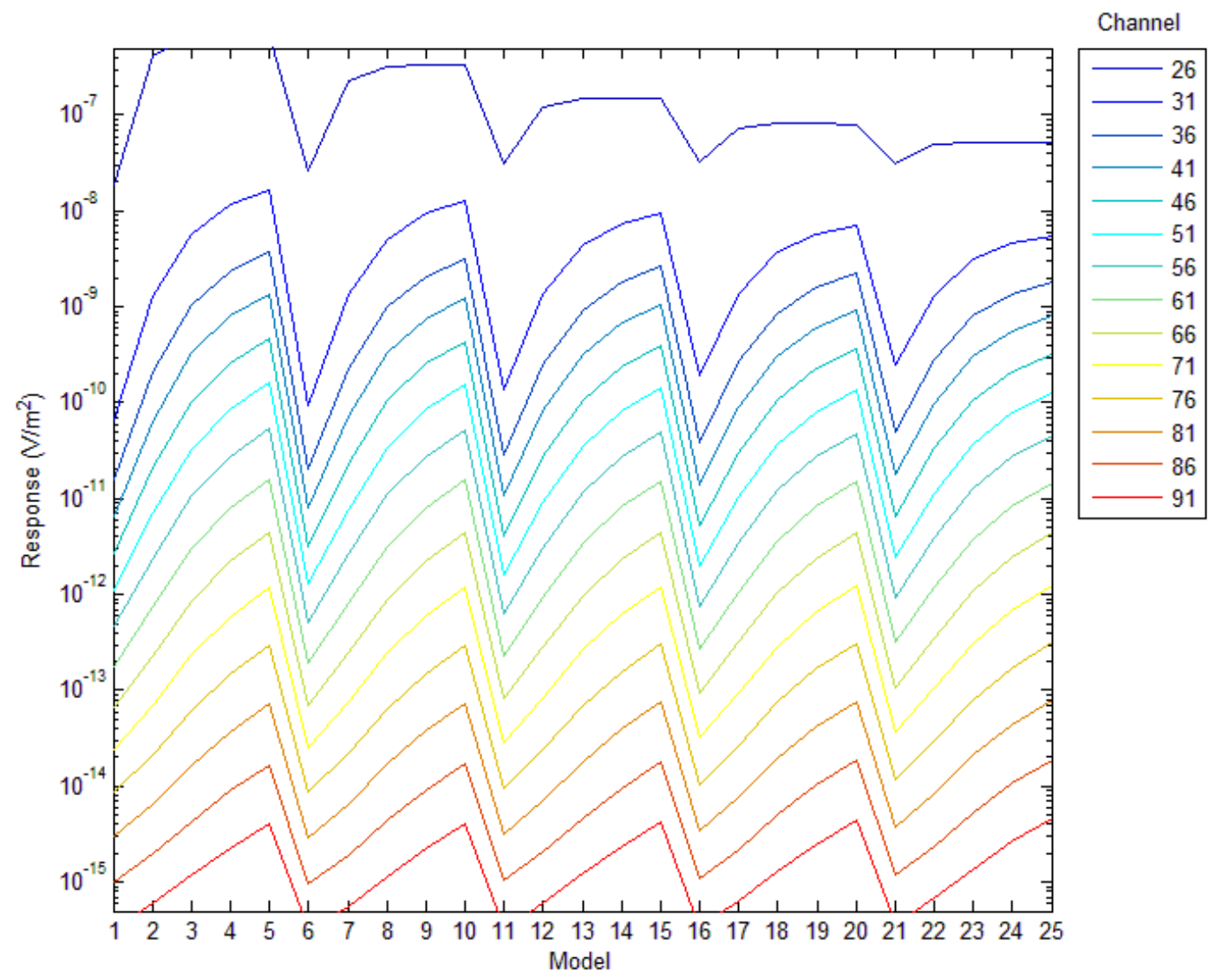

Figure 5.8. Response of multiple channels for graphite model C. Every 5 models shares the same graphite layer depth, but with increasing graphite layer thickness. A summary of the depth and thickness of the graphite layer represented on the abscissa is presented in Table 5.2. The model resistivity used is presented in Table 5.3. 
The forward modelling response resulting from models $A, B$, and $C$ are presented in Figure 5.6, Figure 5.7, and Figure 5.8, respectively. In general, the earlier channels have a higher response, and there is a significant drop in response at every change in depth. For a constant depth, the response tends to exhibit diminishing return, where the response has a smaller increase for each successive increase in thickness. However, some channels exhibit a plateau for the earlier time channels in the more conductive models.

When comparing the three figures, we can analyse the effect of the conductivity of the graphite layer. For a given depth, as the conductivity increases, a plateau is reached more easily. At these plateaus, the decay curves are very similar regardless of layer thickness, thus they are insensitive to layer thickness. This makes inverting data using these decay curves ambiguous. 


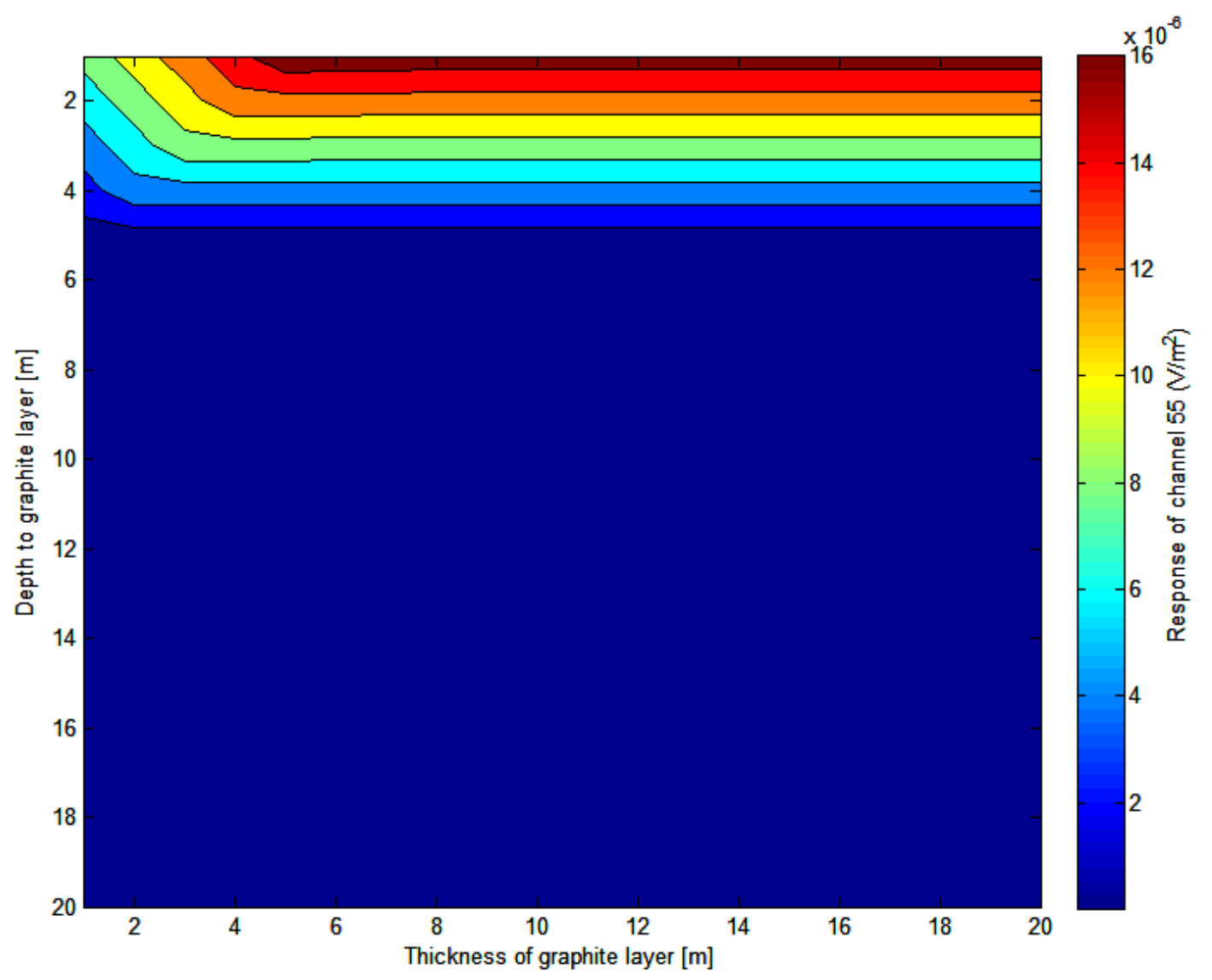

Figure 5.9. Contour plot of the response of model A versus the depth and thickness of the graphite layer. 


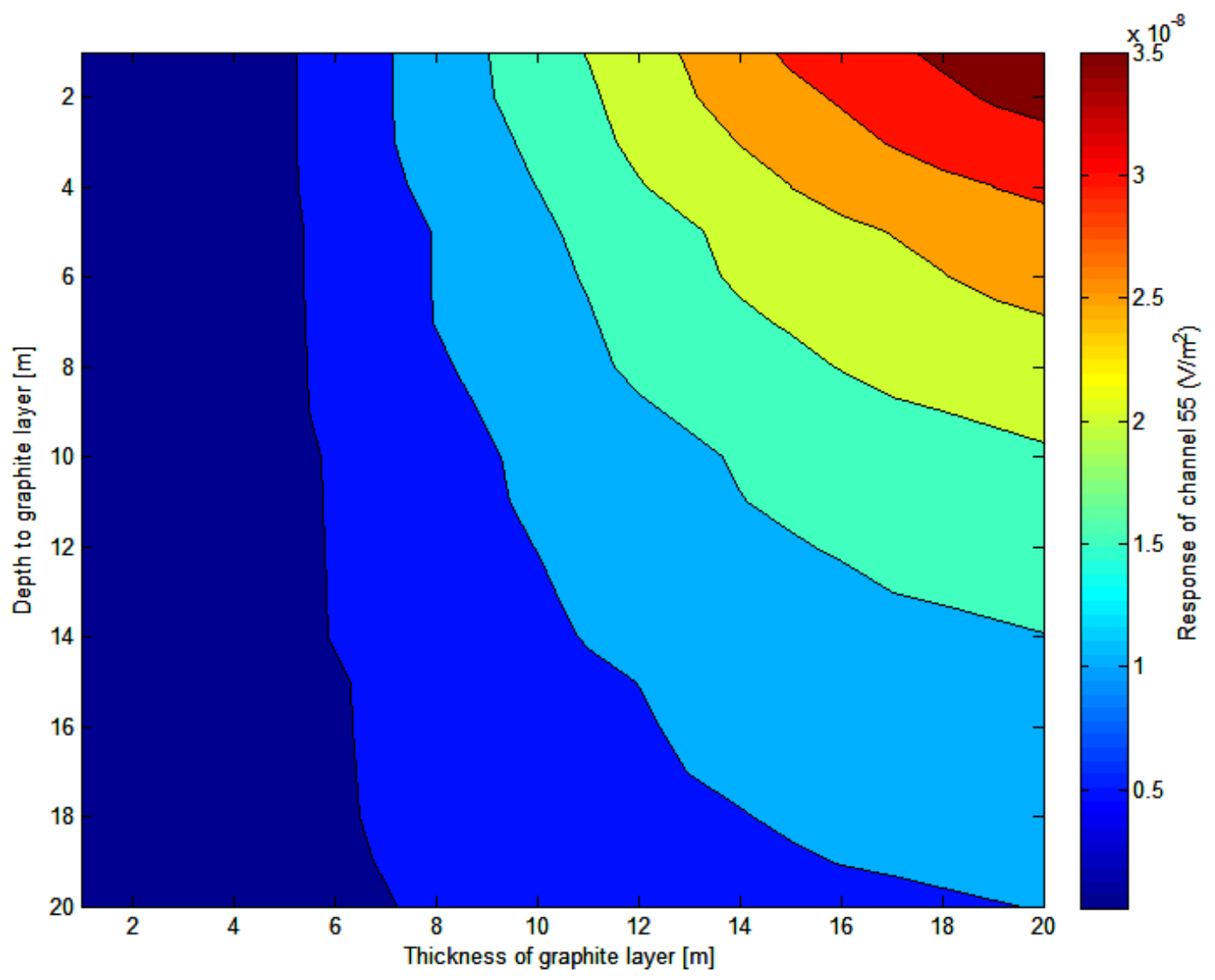

Figure 5.10. Contour plot of the response of model $\mathrm{C}$ versus the depth and thickness of the graphite layer. 
The effects of depth and thickness of the graphite layer on the response of the IMAGEM system can also be visualized by plotting the response as a function of depth and thickness and interpolating to create a contour plot. This is presented in Figure 5.9 for graphite model A and Figure 5.10 for graphite model C.

For model A, the response is only a function of thickness for approximately the first 5 metres of thickness and 5 metres of depth. The response increases with depth for the first 5 metres of depth for all thicknesses, after which the response is approximately constant. The response also increases with thickness for only the first 5 metres of depth, after which the response is constant.

For model C, the response is a function of both depth and thickness for all depths and thicknesses. This relationship appears to be fairly equal and constant with respect to the two parameters. There is some flattening of the response at small thicknesses, where the response appears to be more strongly influenced by depth. Similarly, there is flattening at high thicknesses as well, where the response is less influenced by depth than by thickness. However, this relationship can be more skewed depending on which channel is being observed. For example, looking at Figure 5.8, the relationship of depth versus thickness for the late time channels is linear; the response of channel 91 increases linearly with increasing model (and thus increasing graphite layer thickness). But for the earlier time channels, there is the 'arching' of the response, suggesting the relationship is not linear, like for channel 31, where the response increases by its maximum amount for the first increase in thickness (model 1 to model 2, where the 
depth is constant but the thickness increases by 4 metres), and the next largest increase is between model 2 to model 3 (an increase in 5 metres of thickness), and the increase in response continues to diminish relative to the previous until the depth of the graphite layer changes (between model 5 and model 6), and the effect is repeated for the next depth. 


\subsection{Inversion}

To test the inversion capabilities of AarhusInv, the program is used to invert synthetic decay curves that have been modified by adding noise. The inversion is expected to output the same (or very close) model as the forward model used to create the decay curve.

The noise added to the synthetic forward model was obtained by calculating the standard deviation of a stack of measurements by the IMAGEM system in the field and is further explained in Section 6.1.1. The forward modelling decay curves with added synthetic noise are presented in Figure 5.11. These decay curves are the input for the inversion. A summary of the results of the inversions is presented in Table 5.4. 


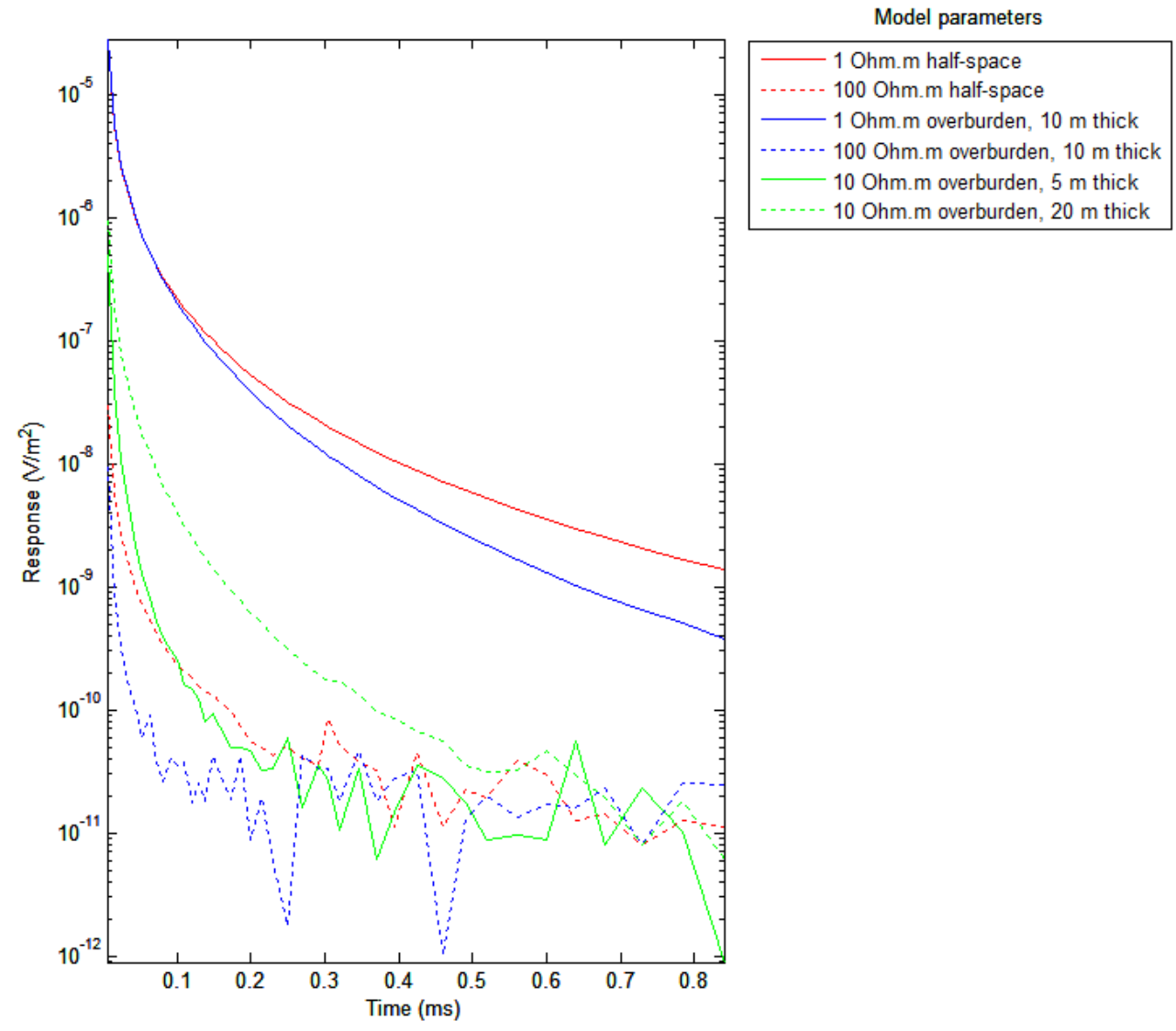

Figure 5.11. The forward model decay curves with added synthetic noise. The synthetic noise is based on calculated noise measured with the IMAGEM system. 
Table 5.4. Summary of the results of inverting synthetic data.

\begin{tabular}{|c|c|c|c|c|}
\hline & & \multicolumn{3}{|c|}{ Half-space model A } \\
\hline & & Forward model & Initial guess model & Inverted model \\
\hline \multirow{2}{*}{ Resistivity $(\Omega \cdot m)$} & Layer 1 & 1 & 5 & 1 \\
\hline & Layer 2 & 1 & 50 & 1.1 \\
\hline \multirow{2}{*}{ Thickness (m) } & Layer 1 & $\infty$ & 10 & 24.3 \\
\hline & Layer 2 & $\mathrm{~N} / \mathrm{A}$ & $\infty$ & $\infty$ \\
\hline \multicolumn{2}{|c|}{ \# of iterations } & N/A & $\mathrm{N} / \mathrm{A}$ & 15 \\
\hline \multicolumn{2}{|c|}{ Start residual } & N/A & $\mathrm{N} / \mathrm{A}$ & 77.476 \\
\hline \multicolumn{2}{|c|}{ End residual } & N/A & $\mathrm{N} / \mathrm{A}$ & 0.086 \\
\hline & & \multicolumn{3}{|c|}{ Half-space model B } \\
\hline & & Forward model & Initial guess model & Inverted model \\
\hline \multirow{2}{*}{ Resistivity $(\Omega \cdot m)$} & Layer 1 & 100 & 100 & 23.8 \\
\hline & Layer 2 & 100 & 1,000 & 102.5 \\
\hline \multirow{2}{*}{ Thickness (m) } & Layer 1 & $\infty$ & 10 & 0.42 \\
\hline & Layer 2 & $N / A$ & $\infty$ & $\infty$ \\
\hline \multicolumn{2}{|c|}{ \# of iterations } & $\mathrm{N} / \mathrm{A}$ & $\mathrm{N} / \mathrm{A}$ & 9 \\
\hline \multicolumn{2}{|c|}{ Start residual } & $\mathrm{N} / \mathrm{A}$ & $\mathrm{N} / \mathrm{A}$ & 66.122 \\
\hline \multicolumn{2}{|c|}{ End residual } & & $\mathrm{N} / \mathrm{A}$ & 0.454 \\
\hline & & \multicolumn{3}{|c|}{ Overburden resistivity model A } \\
\hline & & Forward model & Initial guess model & Inverted model \\
\hline \multirow{2}{*}{ Resistivity $(\Omega \cdot m)$} & Layer 1 & 1 & 50 & 1 \\
\hline & Layer 2 & 1,000 & 500 & 952.9 \\
\hline \multirow{2}{*}{ Thickness (m) } & Layer 1 & 10 & 10 & 10.1 \\
\hline & Layer 2 & $\infty$ & $\infty$ & $\infty$ \\
\hline \multicolumn{2}{|c|}{ \# of iterations } & $\mathrm{N} / \mathrm{A}$ & $\mathrm{N} / \mathrm{A}$ & 10 \\
\hline \multicolumn{2}{|c|}{ Start residual } & $\mathrm{N} / \mathrm{A}$ & $\mathrm{N} / \mathrm{A}$ & 164.512 \\
\hline \multicolumn{2}{|c|}{ End residual } & $\mathrm{N} / \mathrm{A}$ & $\mathrm{N} / \mathrm{A}$ & 0.072 \\
\hline & & \multicolumn{3}{|c|}{ Overburden resistivity model B } \\
\hline & & Forward model & Initial guess model & Inverted model \\
\hline \multirow{2}{*}{ Resistivity $(\Omega \cdot m)$} & Layer 1 & 100 & 50 & 115.9 \\
\hline & Layer 2 & 1,000 & 500 & 1026.2 \\
\hline \multirow{2}{*}{ Thickness (m) } & Layer 1 & 10 & 10 & 12 \\
\hline & Layer 2 & $\infty$ & $\infty$ & $\infty$ \\
\hline \multicolumn{2}{|c|}{ \# of iterations } & $\mathrm{N} / \mathrm{A}$ & $\mathrm{N} / \mathrm{A}$ & 9 \\
\hline \multicolumn{2}{|c|}{ Start residual } & $\mathrm{N} / \mathrm{A}$ & $\mathrm{N} / \mathrm{A}$ & 26.115 \\
\hline \multicolumn{2}{|c|}{ End residual } & $\mathrm{N} / \mathrm{A}$ & $N / A$ & 0.476 \\
\hline
\end{tabular}




\begin{tabular}{|c|c|c|c|c|}
\hline & \multicolumn{3}{|c|}{ Overburden thickness model A } \\
\hline & & Forward model & Initial guess model & Inverted model \\
\hline \multirow{2}{*}{ Resistivity $(\Omega \cdot m)$} & Layer 1 & 10 & 50 & 12.1 \\
\hline & Layer 2 & 1,000 & 500 & 1532.3 \\
\hline \multirow{2}{*}{ Thickness (m) } & Layer 1 & 5 & 10 & 6.4 \\
\hline & Layer 2 & $\infty$ & $\infty$ & $\infty$ \\
\hline \multicolumn{2}{|c|}{ \# of iterations } & $\mathrm{N} / \mathrm{A}$ & $\mathrm{N} / \mathrm{A}$ & 14 \\
\hline \multicolumn{2}{|c|}{ Start residual } & N/A & $\mathrm{N} / \mathrm{A}$ & 24.097 \\
\hline \multicolumn{2}{|c|}{ End residual } & N/A & $\mathrm{N} / \mathrm{A}$ & 1.686 \\
\hline & & \multicolumn{3}{|c|}{ Overburden thickness model B } \\
\hline & & Forward model & Initial guess model & Inverted model \\
\hline \multirow{2}{*}{ Resistivity $(\Omega \cdot m)$} & Layer 1 & 10 & 50 & 10.1 \\
\hline & Layer 2 & 1,000 & 500 & 1104.4 \\
\hline \multirow{2}{*}{ Thickness (m) } & Layer 1 & 20 & 10 & 20.3 \\
\hline & Layer 2 & $\infty$ & $\infty$ & $\infty$ \\
\hline \multicolumn{2}{|c|}{ \# of iterations } & $\mathrm{N} / \mathrm{A}$ & N/A & 10 \\
\hline \multicolumn{2}{|c|}{ Start residual } & N/A & $\mathrm{N} / \mathrm{A}$ & 80.685 \\
\hline \multicolumn{2}{|c|}{ End residual } & $\mathrm{N} / \mathrm{A}$ & $\mathrm{N} / \mathrm{A}$ & 0.061 \\
\hline
\end{tabular}


[A]

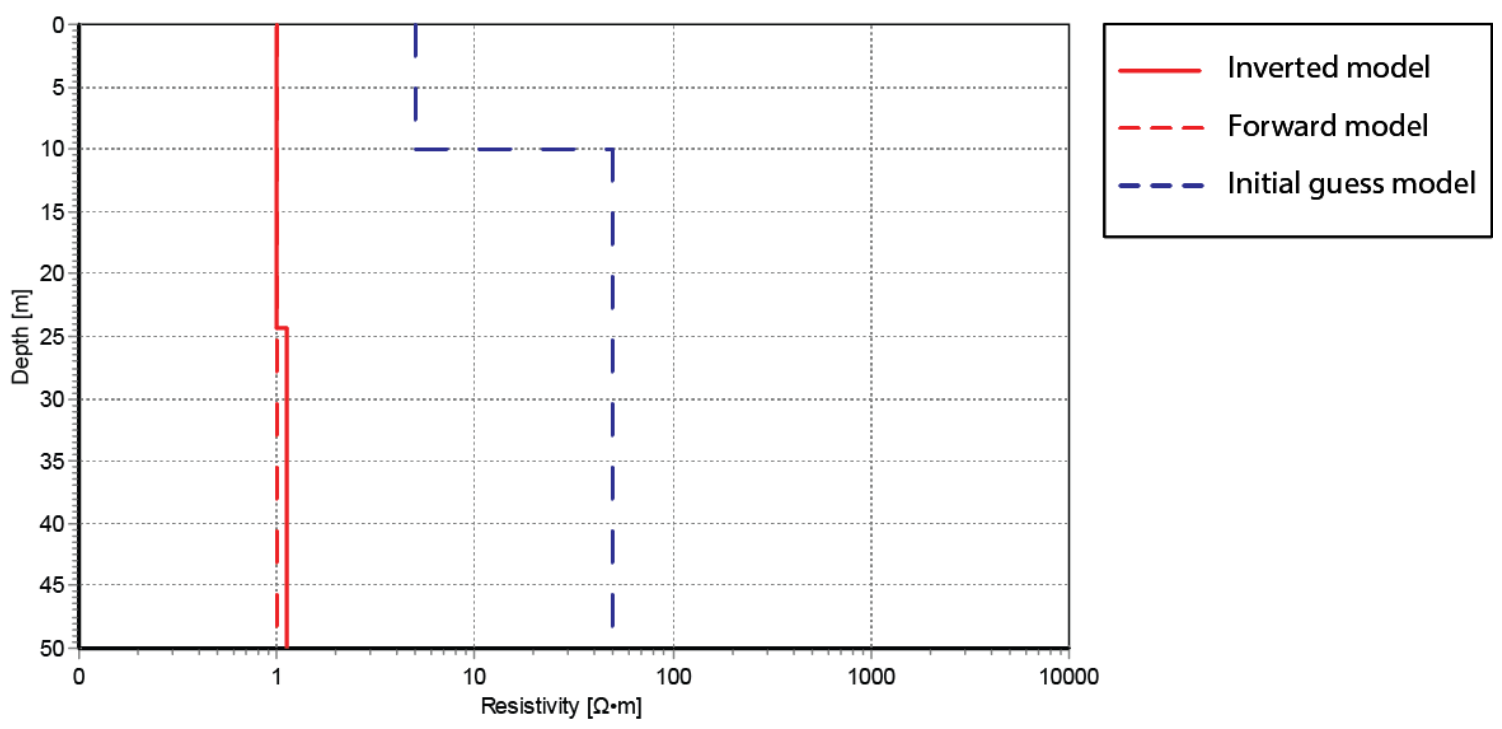

[B]

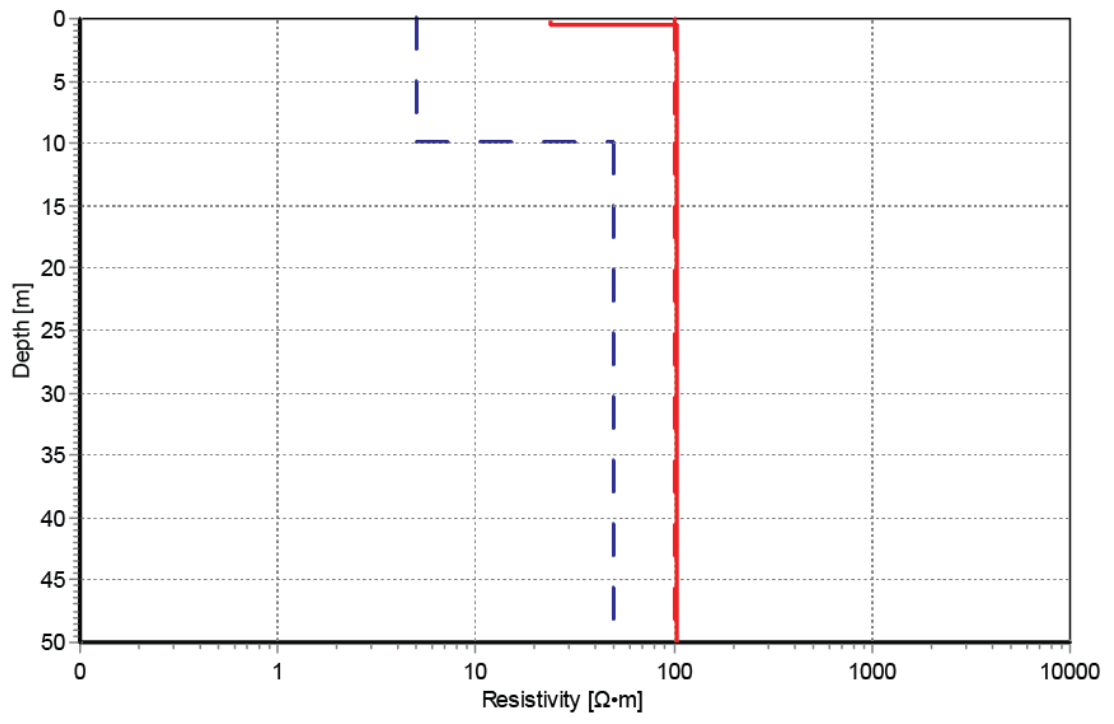

Figure 5.12. Result of the inversion of the half-space models. [A] Inversion of half-space model with a resistivity of $1 \Omega \cdot \mathrm{m}$. [B] Inversion of half-space with a resistivity of $100 \Omega \cdot \mathrm{m}$. 
Model parameters

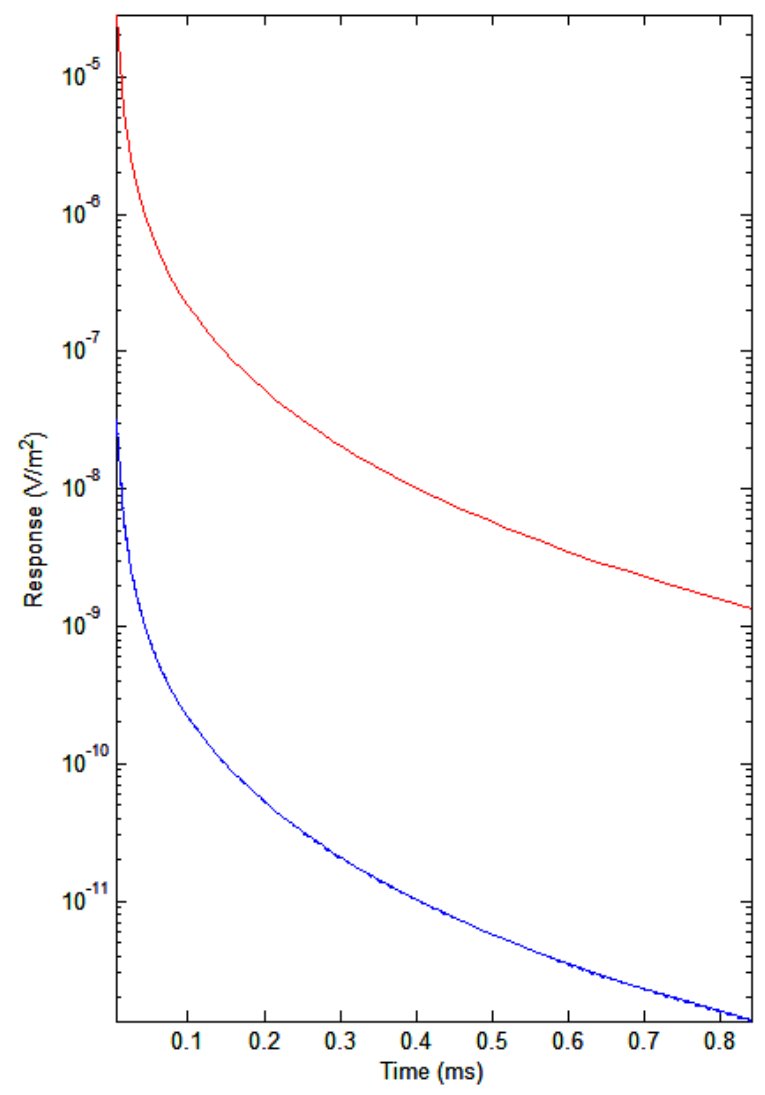

\begin{tabular}{l} 
_ Forward model: $1 \mathrm{Ohm} . \mathrm{m}$ half-space \\
_. Inverted model: $1 \mathrm{Ohm} . \mathrm{m}$ half-space \\
- Forward model: $100 \mathrm{Ohm} . \mathrm{m}$ half-space \\
\hline - Inverted model: $100 \mathrm{Ohm} . \mathrm{m}$ half-space
\end{tabular}

Figure 5.13. Comparing the decay curve achieved by the forward model with the decay curve achieved by forward modelling of the inverted model for the half-space models. 
The results of inverting the half-space models are presented in Figure 5.12. The dotted red line represents the parameters of the model used to create the decay curve (the 'true' model that the inversion program should match). The dotted blue line represents the parameters of the initial guess model, which is a user chosen model from which the program will begin its iterative process. In this example, a two-layer model with a resistivity of $5 \Omega \cdot \mathrm{m}$ and $50 \Omega \cdot \mathrm{m}$ for the first and second layers, respectively, was chosen. This was done because the program appeared to output much better results when using a two-layer model, and where the resistivity of the second layer was greater than the first. The solid red line represents the inverted model, calculated by the software based on the data (the forward-modelled decay curve) and the initial guess model. In Figure 5.12A, the resistivity of the inverted model matches the forward model very closely. A slight deviation occurs at a depth of $24.3 \mathrm{~m}$. For Figure 5.12B, similar results are observed. One significant difference is present: a very thin $(0.4 \mathrm{~m})$ layer has been introduced near the surface, with a resistivity of $23.8 \Omega \cdot \mathrm{m}$. This layer, however, is so thin that its effects are almost negligible.

By running a forward model using the parameters of the inverted model, we can compare the results of the inversion with the decay curve from the original model. For the half-space models, these are presented in Figure 5.13. For both models, both curves follow each other almost perfectly, meaning the program was able to invert the data and achieve a model very close to the model used to generate the data, for this very simple example. 
[A]

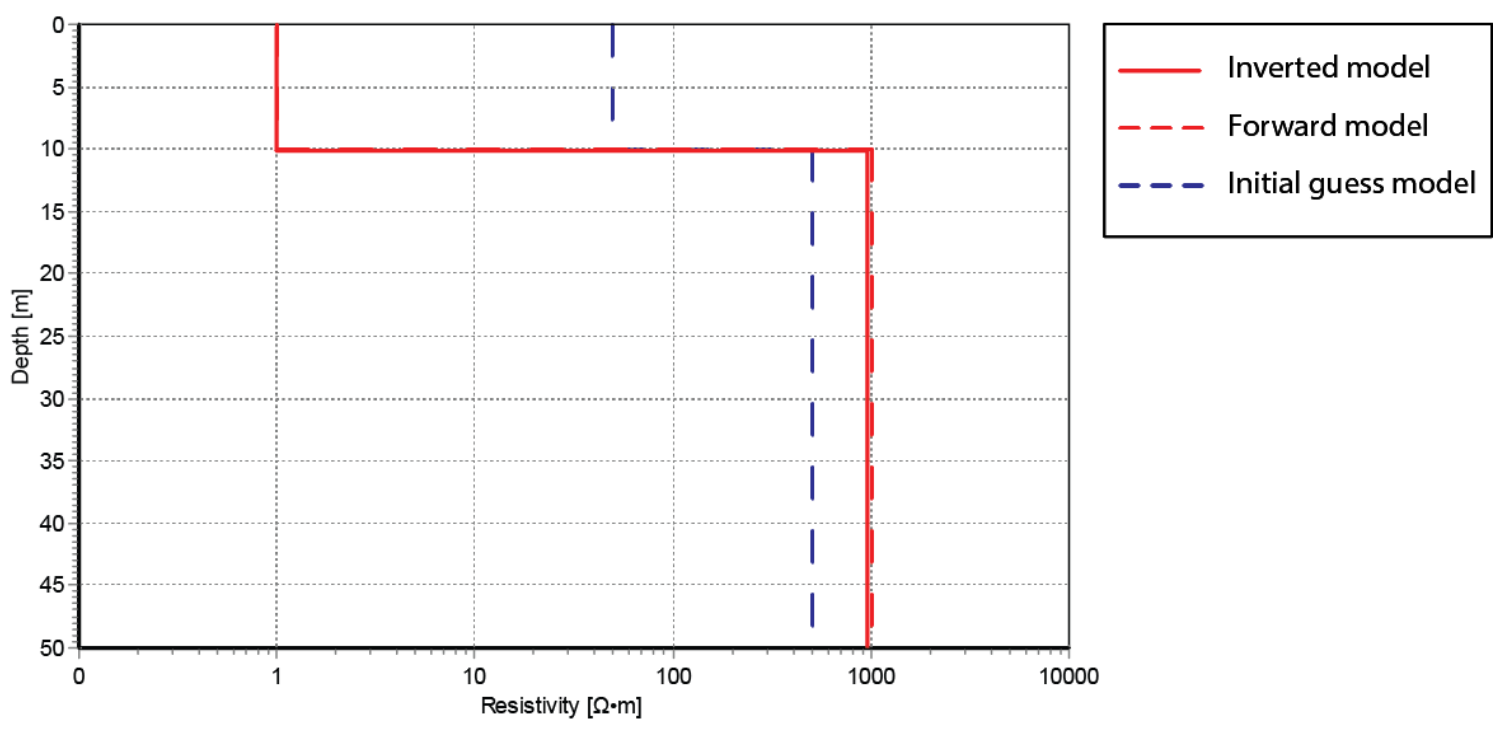

[B]

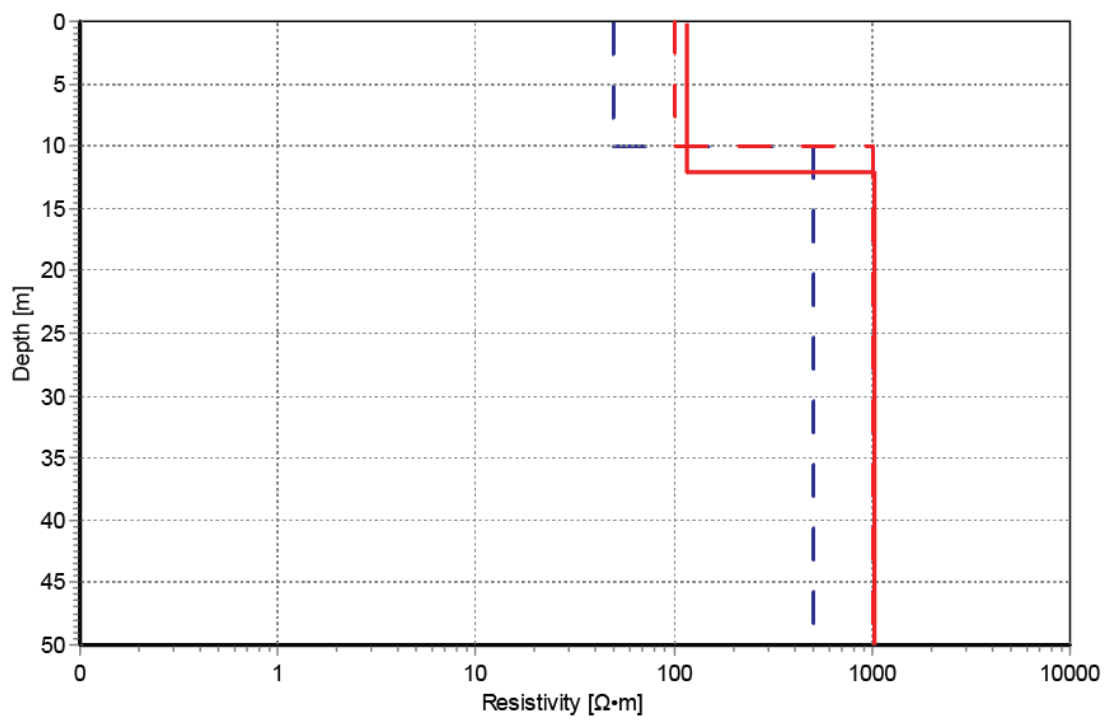

Figure 5.14. Result of the inversion of the overburden resistivity models. [A] Inversion of the $1 \Omega \cdot \mathrm{m}$ overburden model over a 1,000 $\Omega \cdot \mathrm{m}$ half-space. [B] Inversion of the $100 \Omega \cdot \mathrm{m}$ overburden model over a $1,000 \Omega \cdot m$ half-space. 


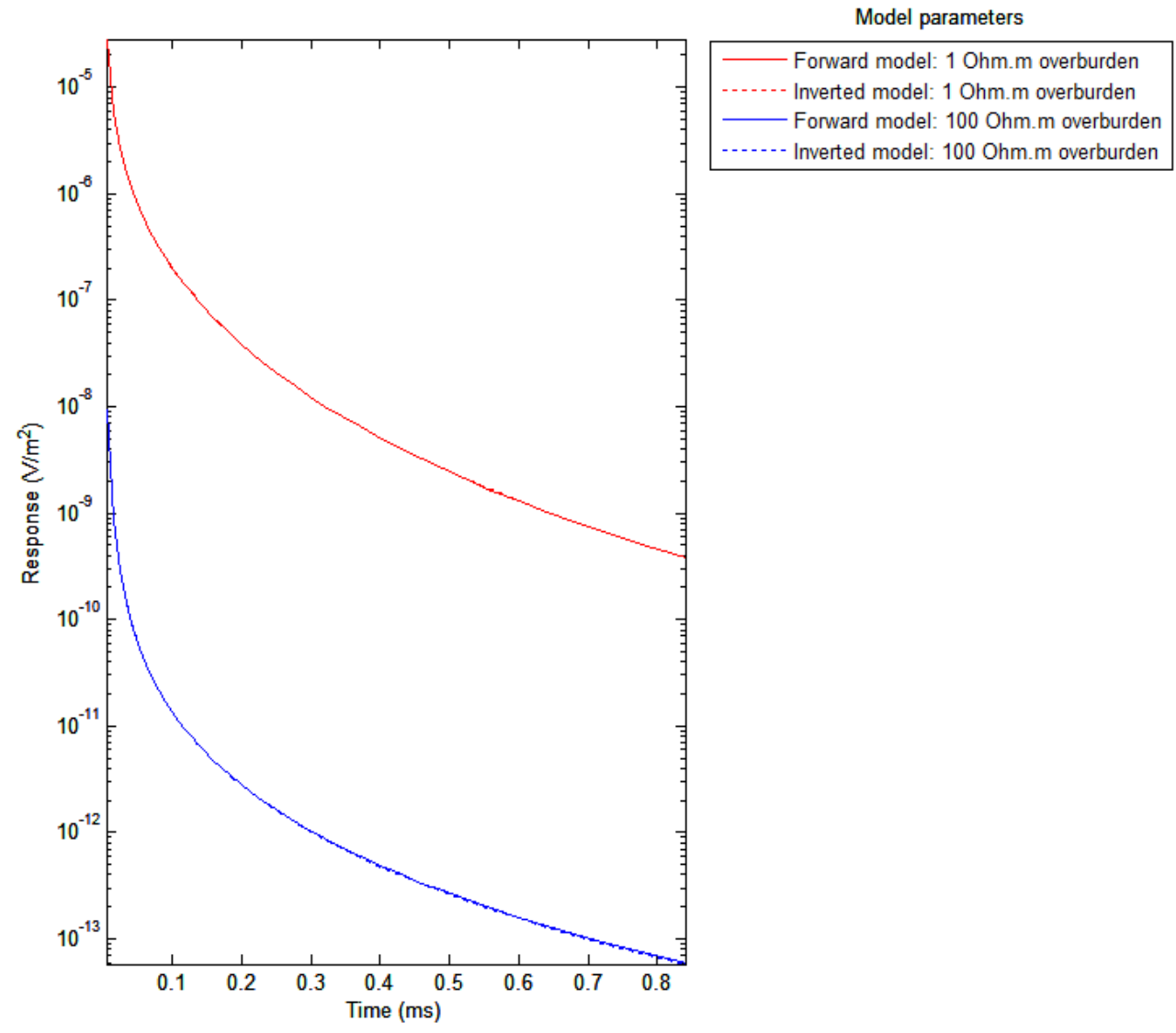

Figure 5.15. Comparing the decay curves calculated using the initial model (solid lines) with the decay curves calculated by forward modelling of the inverted model (dotted lines) for the overburden resistivity models. 
The results of the inversions of the overburden resistivity models are presented in Figure 5.14. In Figure 5.14A the resistivity of the inverted model matches that of the initial model very well. In Figure 5.14B, however, the inverted model does not match quite as well. There is a slight difference in resistivity of the first layer, and a slight difference in the depth of the second layer between the inverted model and the forward model. This difference can be explained in Figure 5.11. The decay curve of model $B$ is very much in the noise, while the amplitude of the curve of model $A$ is much higher and is not affected by the noise.

The comparison between the decay curves of the inverted model and the original forward model is presented in Figure 5.15. Similarly as the half-space models, both curves match very closely. 
[A]

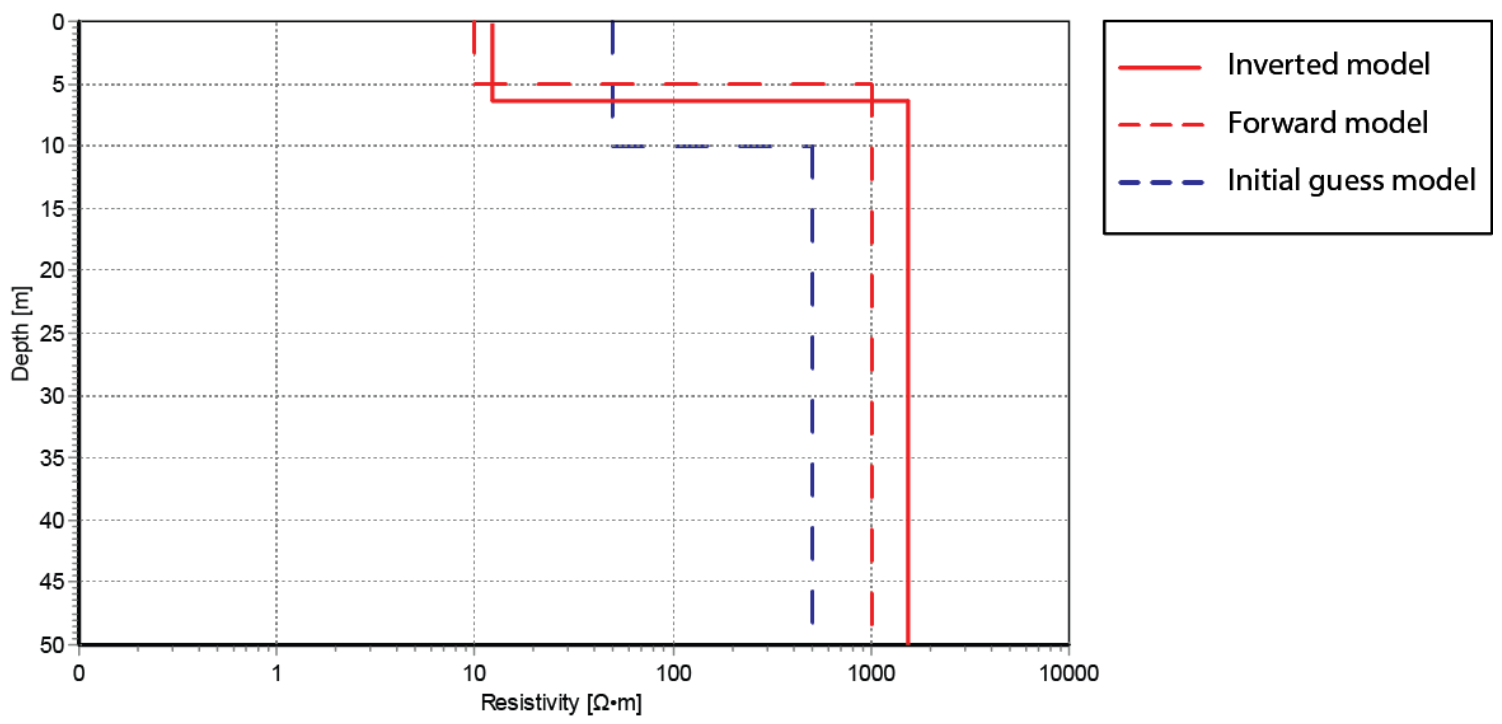

[B]

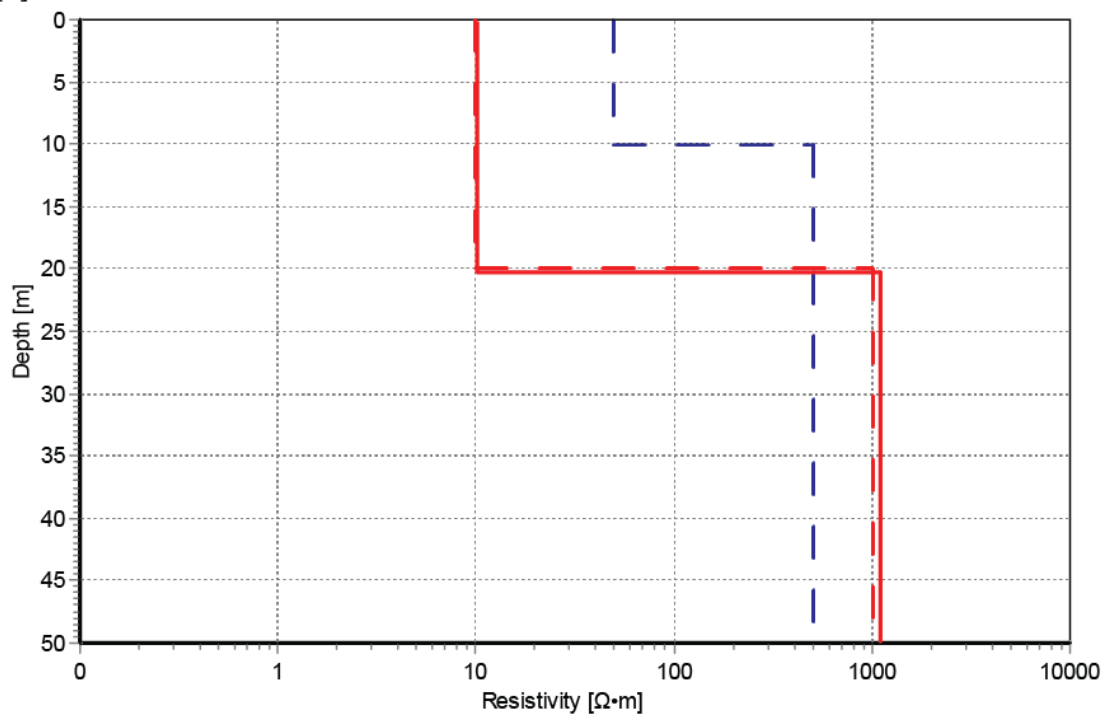

Figure 5.16. Result of the inversion of the overburden thickness models. [A] Inversion of the $5 \mathrm{~m}$ overburden model with a resistivity of $10 \Omega \cdot \mathrm{m}$ over a $1,000 \Omega \cdot \mathrm{m}$ half-space. [B] Inversion of the $20 \mathrm{~m}$ thick overburden model over a 1,000 $\Omega \cdot m$ half-space. 


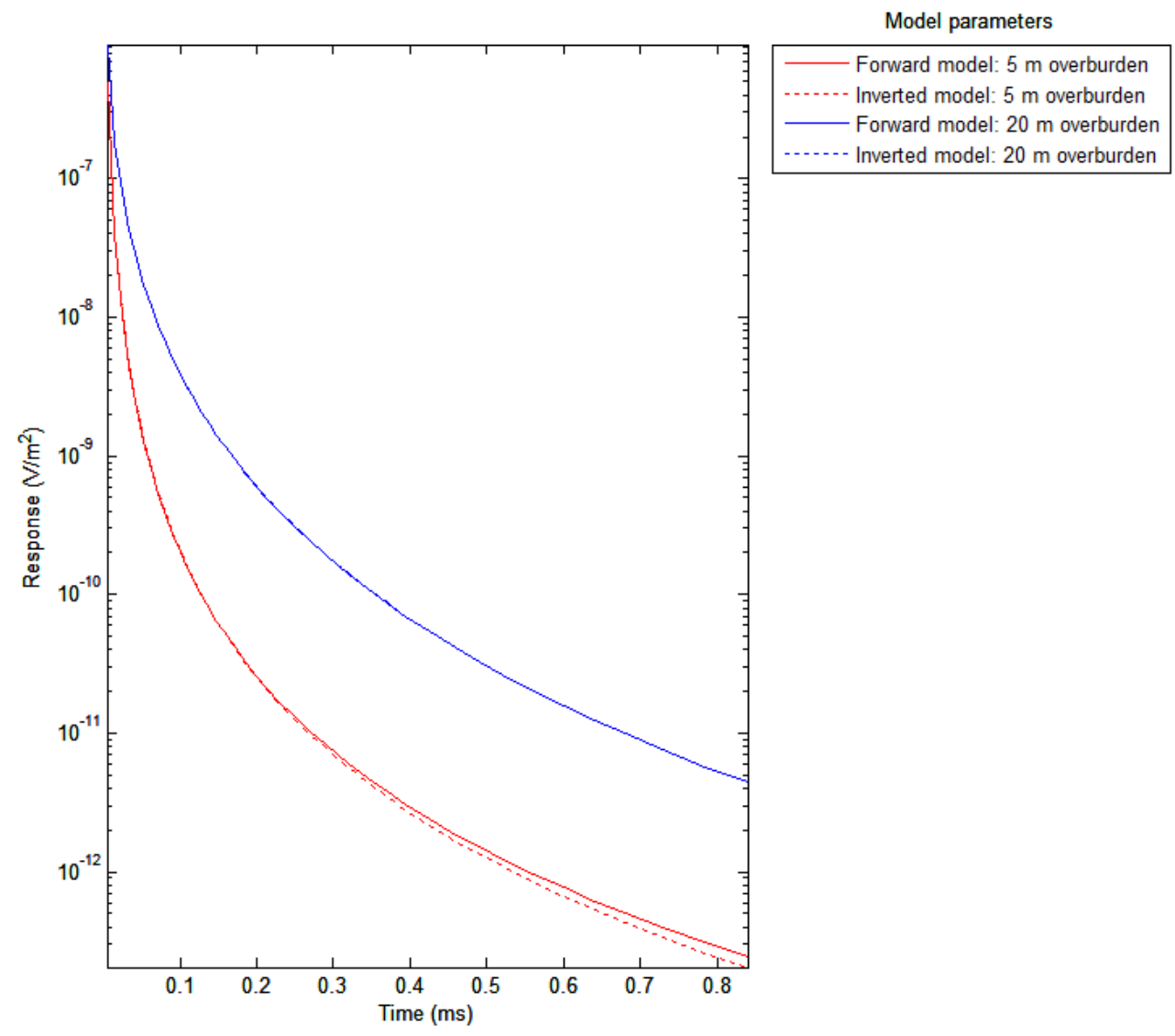

Figure 5.17. Comparing the decay curve achieved by the forward model with the decay curve achieved by forward modelling the inverted model for the overburden thickness models. 
The results of the inversions of the overburden thickness models are presented in Figure 5.16. In Figure 5.16A, the resulting inverted model does not match the forward model as well as the previous models, although it is still close. Looking at Table 5.4, the residual is higher (1.686) than all other models (approximately 0.01-0.5). This is a little surprising as the curve for this model (overburden thickness $A$ ) is not noisier than that of the overburden resistivity model B. This may be explained by the initial guess model. The initial guess model can create large differences in the results of the inversion. A slight tweak of the initial model could cause the inversion to fit much better and with a much lower residual. The results of inverting overburden thickness model B is presented in Figure 5.16B. This inversion fits the forward model very well.

The comparison between the decay curves of the inverted model and the initial model is presented in Figure 5.17. As expected, the decay curves of model A does not match quite as well as the other curves. Model B fits very well. 


\section{Case studies}

Three case studies are presented to demonstrate the capabilities of the IMAGEM system for graphite exploration. The first case study, Mayo, will pertain to analysing the noise level of the IMAGEM system, and attempting to invert IMAGEM and MaxMin field data, and features a comparison between the survey results of the MaxMin system and IMAGEM system, and their inversion results. The next case study, Matawinie, showcases the high spatial resolution of the IMAGEM system and visualization of field data. The third and last case study, Lac Tétépisca, compares the survey results of the IMAGEM system with that of the MaxMin system.

\subsection{Mayo}

The IMAGEM system was used to perform a survey in the city of Mayo in the Outaouais region of Quebec. The survey was performed along a public road on September $14^{\text {th }} 2014$, and spans $1.1 \mathrm{~km}$ in length. This location is used as a test and calibration site, and has been surveyed with the IMAGEM system a number of times, at different stages of its development. The site features a prominent conductivity anomaly attributed to a graphite deposit at depths between 10 to $15 \mathrm{~m}$ beneath the road, striking perpendicularly. The MaxMin system was also used to perform a survey of the same line, so the results may be compared with the IMAGEM system. Datasets from this survey were chosen to analyse the noise of the IMAGEM system, and to test inversion of field data for both the IMAGEM and MaxMin systems. 


\subsubsection{Noise analysis}

Instrument noise associated with the IMAGEM system can be measured by calculating the variation between measurements made at a static location. This is done by taking a 'stack' of measurements - multiple measurements made at the same location that are not affected by the motion of the system or changes in subsurface conditions - and calculating the average standard deviation of the response. Data from the east-west oriented $1.1 \mathrm{~km}$ long line surveyed with the IMAGEM system is presented in Figure 6.1. Changes in the subsurface are easily observed: there is one large response to the west, which is due to a man-made culvert, and two smaller responses to the east, which are believed to be related to graphite deposits. The rest of the line does not exhibit any anomaly, and therefore the subsurface is believed to be of uniform composition in terms of conductivity.

The measurements used to analyse the noise are enclosed in the red rectangle on Figure 6.1. This dataset contains measurements for channels 25 to 66 , spanning 4 seconds of surveying (31 data points), with no change in geology (Figure 6.2). Small variations in response can be observed for each channel.

The decay curves from Figure 6.2 are stacked and presented in Figure 6.3. The standard deviation appears is highest for channels 31-34, and declines towards later channels. The resulting mean standard deviation across all channels is calculated to be $2.96 \times 10^{-8} \mathrm{~V} / \mathrm{m}^{2}$. This is the level of instrument noise which was used in the inversion study presented in Section 5.2. 


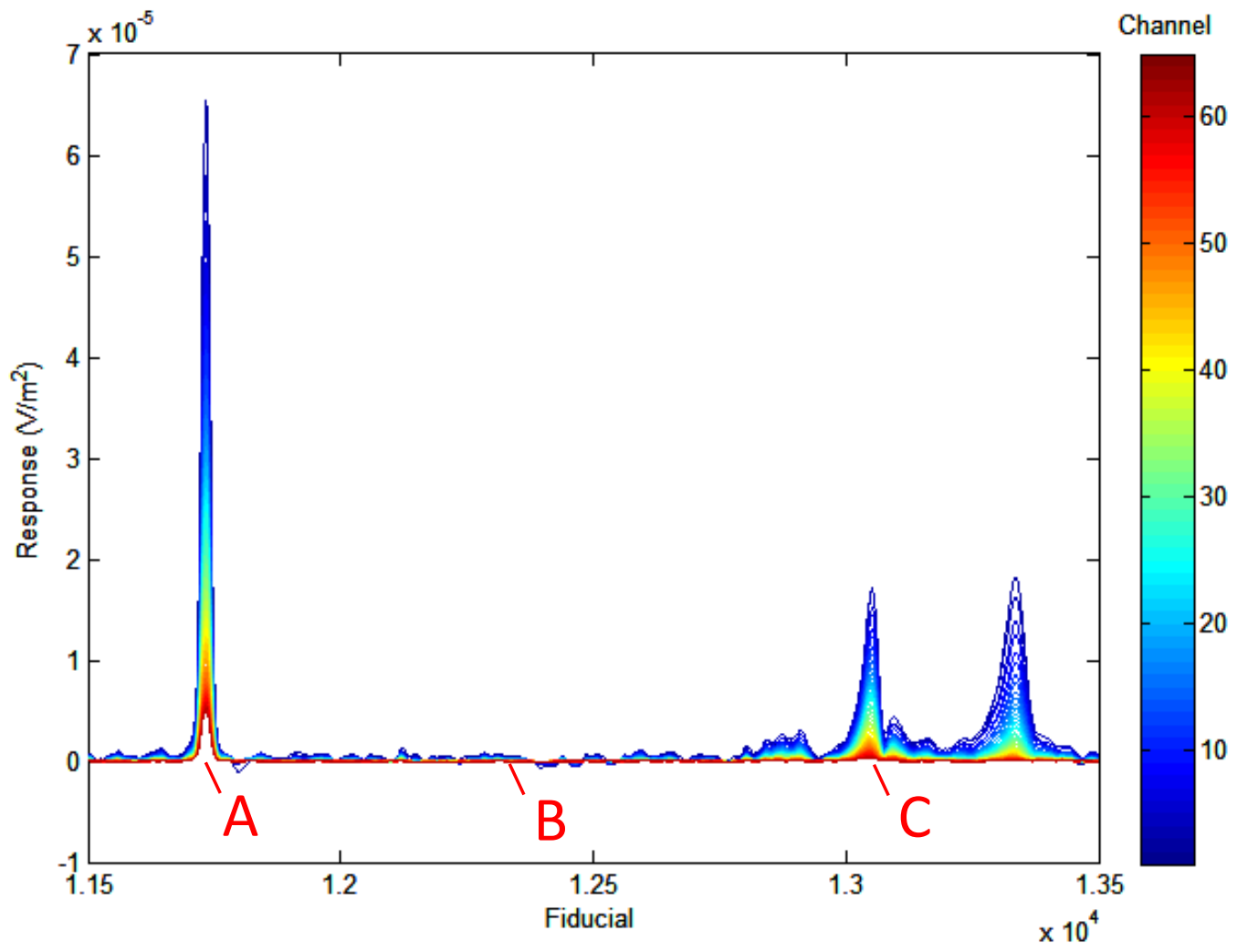

Figure 6.1. Response of the IMAGEM system for a $1.1 \mathrm{~km}$ long section of a survey line at the Mayo calibration site. The red rectangle represents the section used for the noise analysis. 


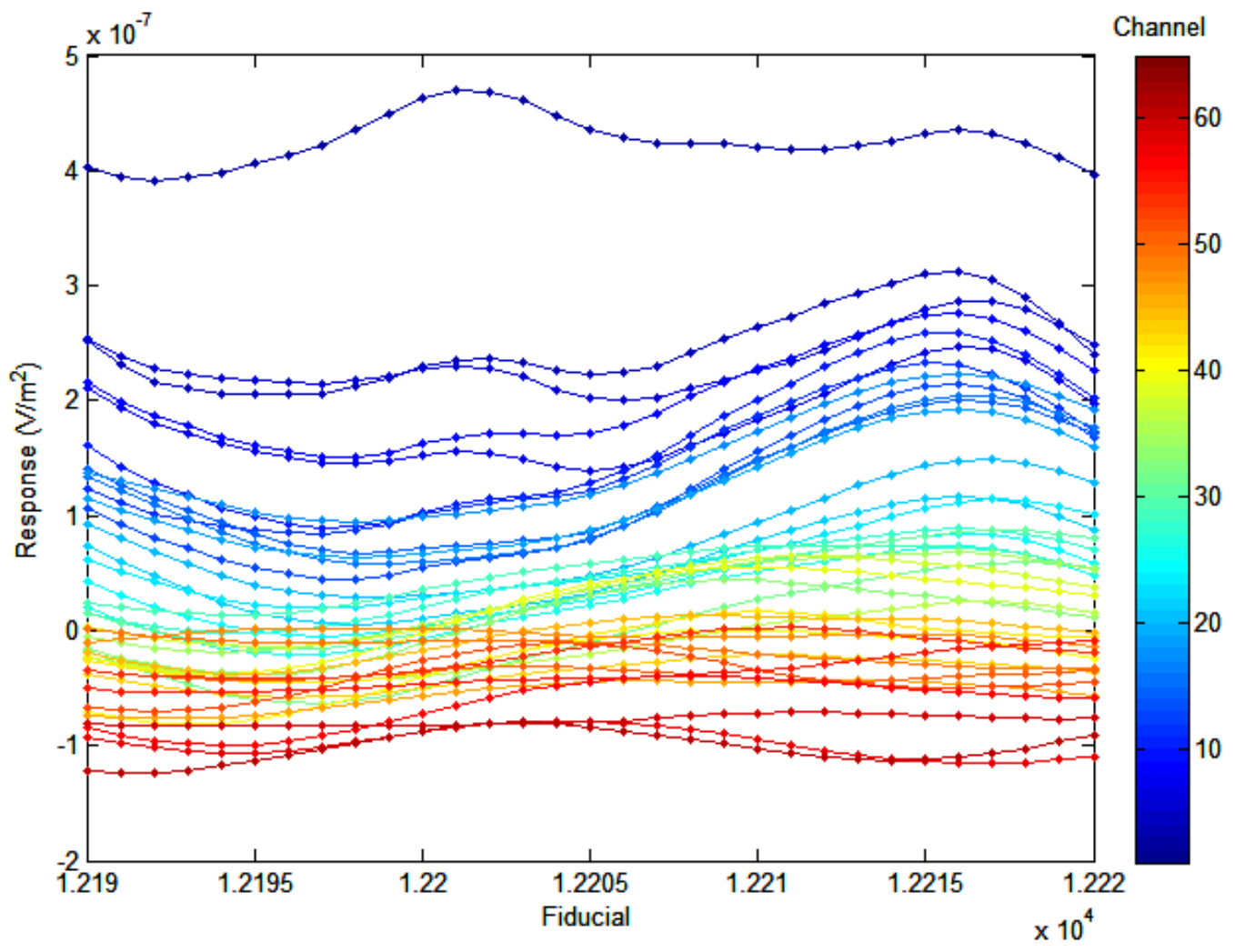

Figure 6.2. Response of the IMAGEM system for the stack of measurements enclosed in the red rectangle in Figure 6.1. 

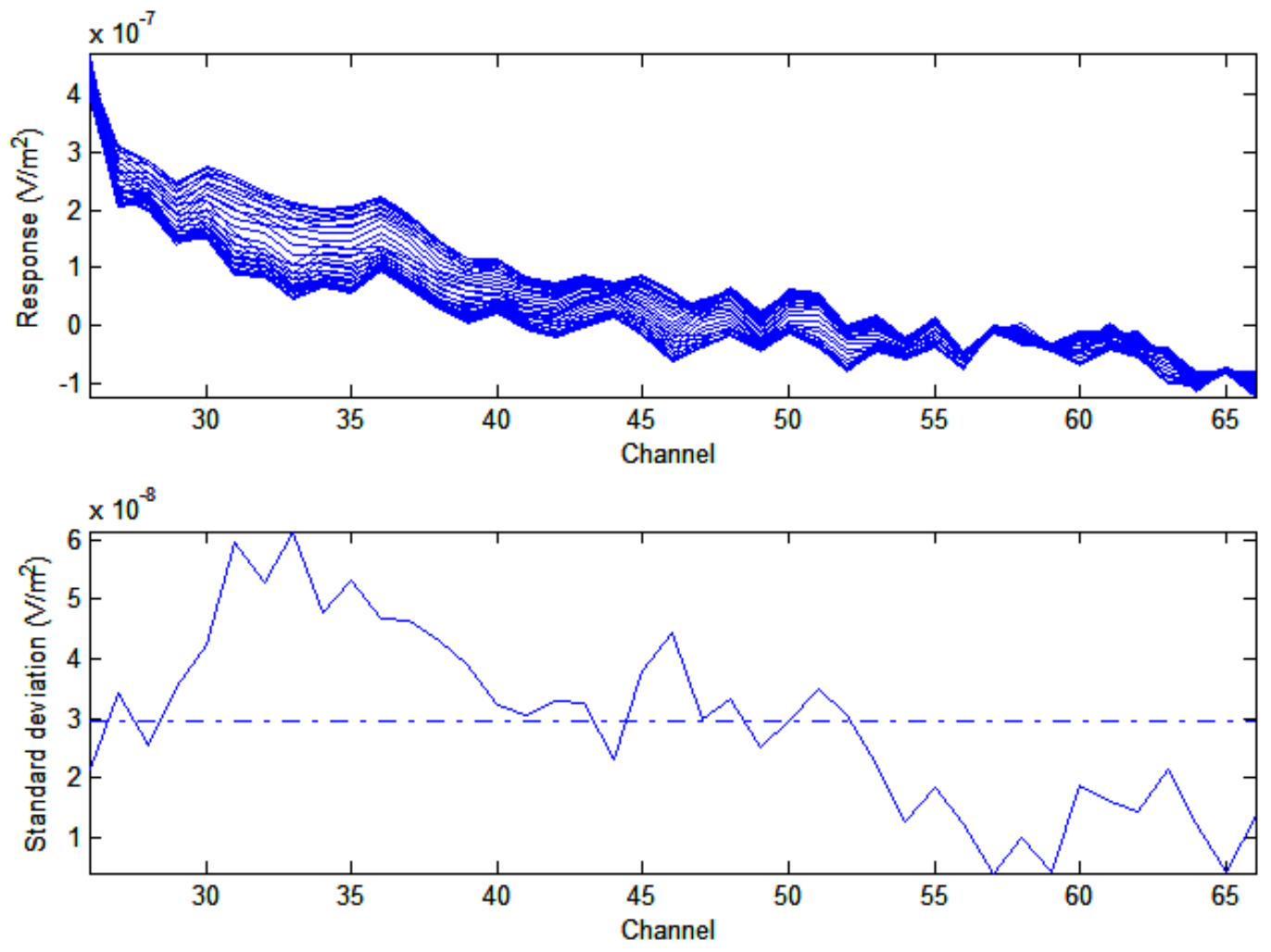

Figure 6.3. (Top) Stack of the decay curves displayed in Figure 6.2. (Bottom) Standard deviation versus channel number. The mean standard deviation (dashed-dotted line) across each channel is $2.96 \times 10^{-8} \mathrm{~V} / \mathrm{m}^{2}$. 


\subsubsection{Inversion}

Field data from the MaxMin and IMAGEM systems were inverted using Aarhusinv. Based on knowledge of the local geology, the expected results of the inversion consists of a conductive overburden of a few metres thick overlaying a resistive basement for the entire length of the survey line other than at the location of the graphite anomaly (point $C$ in Figure 6.1). For this anomaly, the inversion could reveal a more conductive overburden layer, where graphite is disseminated, overlaying a resistive basement. Alternatively, the result of the inversion could be a three-layer model with a conductive overburden layer overlaying a conductive graphite-hosting layer, with a resistive basement beneath.

\subsubsection{Inversion of MaxMin field data}

The data acquired during the MaxMin survey is presented in Figure 6.4. There was a total of 32 stations, approximately spaced $12.5 \mathrm{~m}$ apart using $50 \mathrm{~m}$ coil separation. The graphite anomaly is located approximately between stations 4 and 11 .

Because the MaxMin system measures using pre-defined stations, there are few data points, and is therefore possible to invert the entire line. At each station, there are only 6 data points ( 3 frequencies $\times 2$ types of data (in-phase and quadrature)) to invert. The analysis was also used as an opportunity to see the effects of differing initial guess models on final inversion results. The inversion was performed three times using a different initial guess model each time and the results of the inversion are compared. A summary of the different initial guess models used is presented in Table 6.1. In all cases, a two-layer initial guess model was used. 


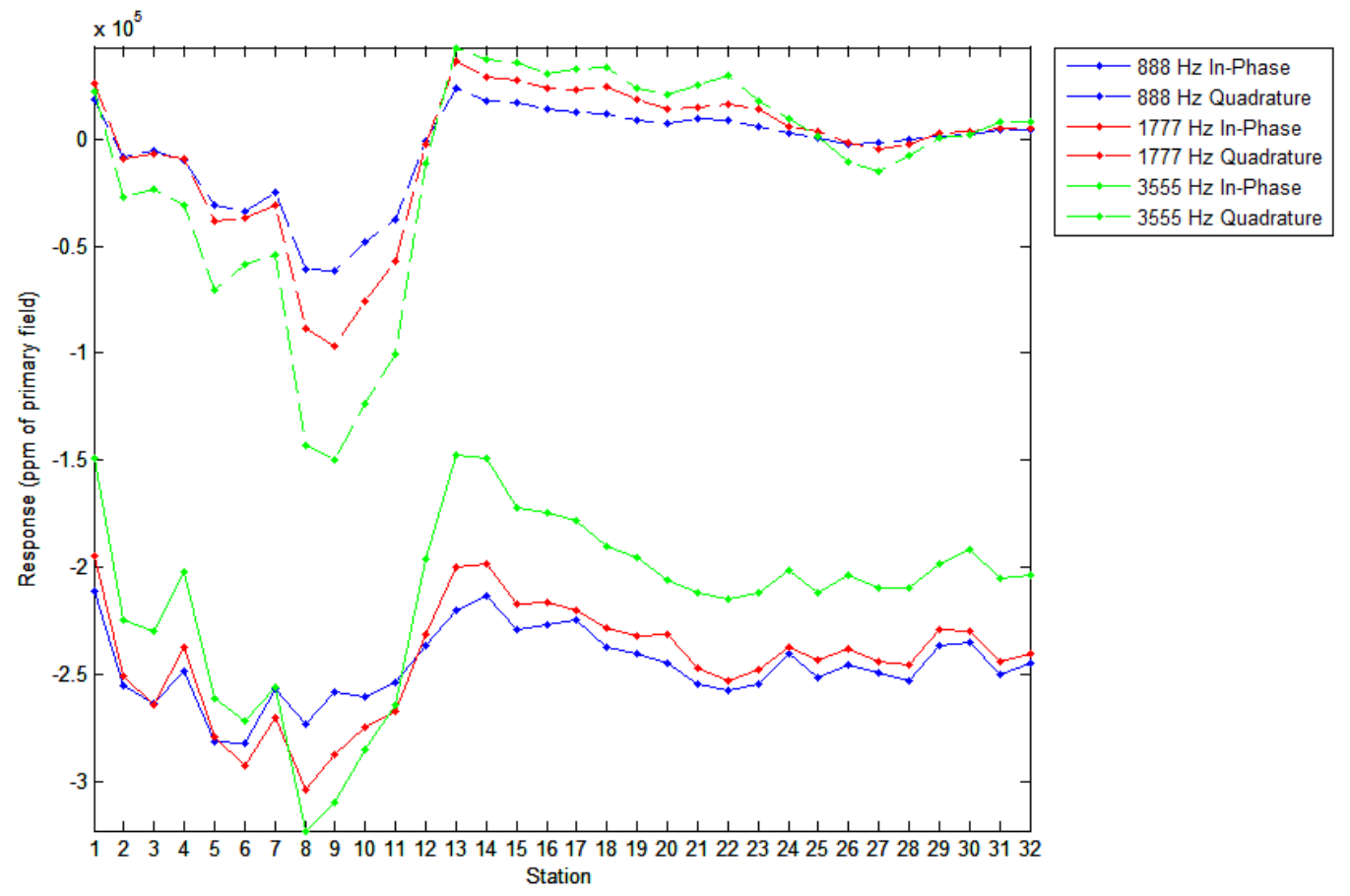

Figure 6.4. Results of the MaxMin survey data. 
Table 6.1. The different initial guess models used for the inversion of the MaxMin data.

\begin{tabular}{|c|c|c|c|}
\hline \multirow{2}{*}{$\begin{array}{c}\text { Initial guess } \\
\text { model }\end{array}$} & \multicolumn{2}{|c|}{ Resistivity $(\mathbf{\Omega} \cdot \mathrm{m})$} & \\
\cline { 2 - 4 } & Layer 1 & Layer 2 & Depth to layer $\mathbf{2}(\mathbf{m})$ \\
\hline $\mathbf{1}$ & 100 & 1,000 & 10 \\
\hline $\mathbf{2}$ & 10 & 100 & 10 \\
\hline $\mathbf{3}$ & 10 & 100 & 1 \\
\hline
\end{tabular}


The results of the inversion using initial guess model 1 is presented in Figure 6.5. Of the three scenarios investigated, this is the scenario for which the initial guess model features the highest resistivities. The residuals are very high, and vary considerably (residuals higher than approximately 5 indicate a very poor fit between field data and inverted data). This indicates that the inversion results are not to be trusted. In particular, at stations 6 to 8 , the inversion algorithm fails to recognize distinct layers (depth to layer $2>250 \mathrm{~m}$ ).

The inversion results using model 2 are presented in Figure 6.6. The residuals are again high, but more consistent. Similarly as model 1 , the software program produces a very thick first layer (>100 m) around the location of the graphite anomaly. However, the resistivity of the first layer does not decrease in this area, which would be needed to suggest a conductive ore body. Although the resisitivity of the second layer decreases, the thickness of the first layer is too thick for the resisitvity of the second layer to be of any significance.

Model 3 is similar to model 2, except that the graphite is hypothesized to be very close to the surface. The results of the inversion using initial guess model 3 are presented in Figure 6.7. As for model 2, the residuals are relatively high but consistent. Unlike the other two models, the depth to layer 2 is consistent, and the largest value is observed at station 6 . The resistivity of layer 1 , however, varies drastically, with the highest resistivity observed between stations 5 and 13 . This is the opposite of what is 
expected as this is where the graphite source body is located. The resistivity of layer 2 is consistent.

This analysis shows that the inversion of the MaxMin data set is very sensitive to the choice of initial guess model. With only 6 points to invert at each station, the inversion is not constrained enough to return valid results. Only one journal article (Attwa et al., 2011) was found in the literature presenting inverted MaxMin data. This article concerned an experimental survey during which 10 MaxMin frequencies were used. There were therefore 20 data points to invert at each station which helped the inversion algorithm to converge to meaningful results in that case.

The expectation of the inversions was for a model consisting of a conductive overburden overlaying a resistive basement for the majority of the survey, and for the location of the graphite anomaly (Stations 4-11 of Figure 6.4) we expected a more conductive first layer, or a greater thickness of the first layer, or even a more conductive second layer. However, the results did not reliably show this. Although there was some increase in layer one thickness observed in models 1 and 2 (Figure 6.5 and Figure 6.6), there lacked the accompanying decrease in resistivity of layer 1 that was expected. The increases in the depth were also much too large to be valid as they greatly surpass the depth of penetration of the MaxMin system for the configuration that was used for the survey. Other results, like model 3 in Figure 6.7, show the opposite of what was expected: an increase in layer one resistivity at the location of the graphite anomaly. The best (lowest) residuals obtained were approximately 5.0. This is much higher than 
the residuals of the synthetic inversion ( 0.05 at best) and on par with that of the IMAGEM field data inversion (3.2 at best).

The problems encountered may have been caused by the improper use of 1D inversion in a situation that merits 2D or 3D inversion. In the case of the MaxMin system, the data is positive when the subsurface is more conductive than the calibration site (which is resistive). However, when a conductive body is thinner (horizontally) than the transmitter-receiver separation, and the profile is perpendicular to the structure, then the anomaly observed in the data is U-shaped and negative, as is seen in the MaxMin results (Figure 6.4). In such a situation, 2D or 3D inversion must be used. For ground systems in general, the footprint of the system must be smaller than the anomaly for 1D inversion to be justified. In the case of the IMAGEM system, 1D inversion was used even where the footprint was larger than the anomaly, such as in the case of the man-made culvert (point A, Figure 6.8) and possibly the graphite deposit (point C, Figure 6.8). At these two locations, 2D or 3D inversion should have been used because the observed data at a single point is affected by the adjacent material. 

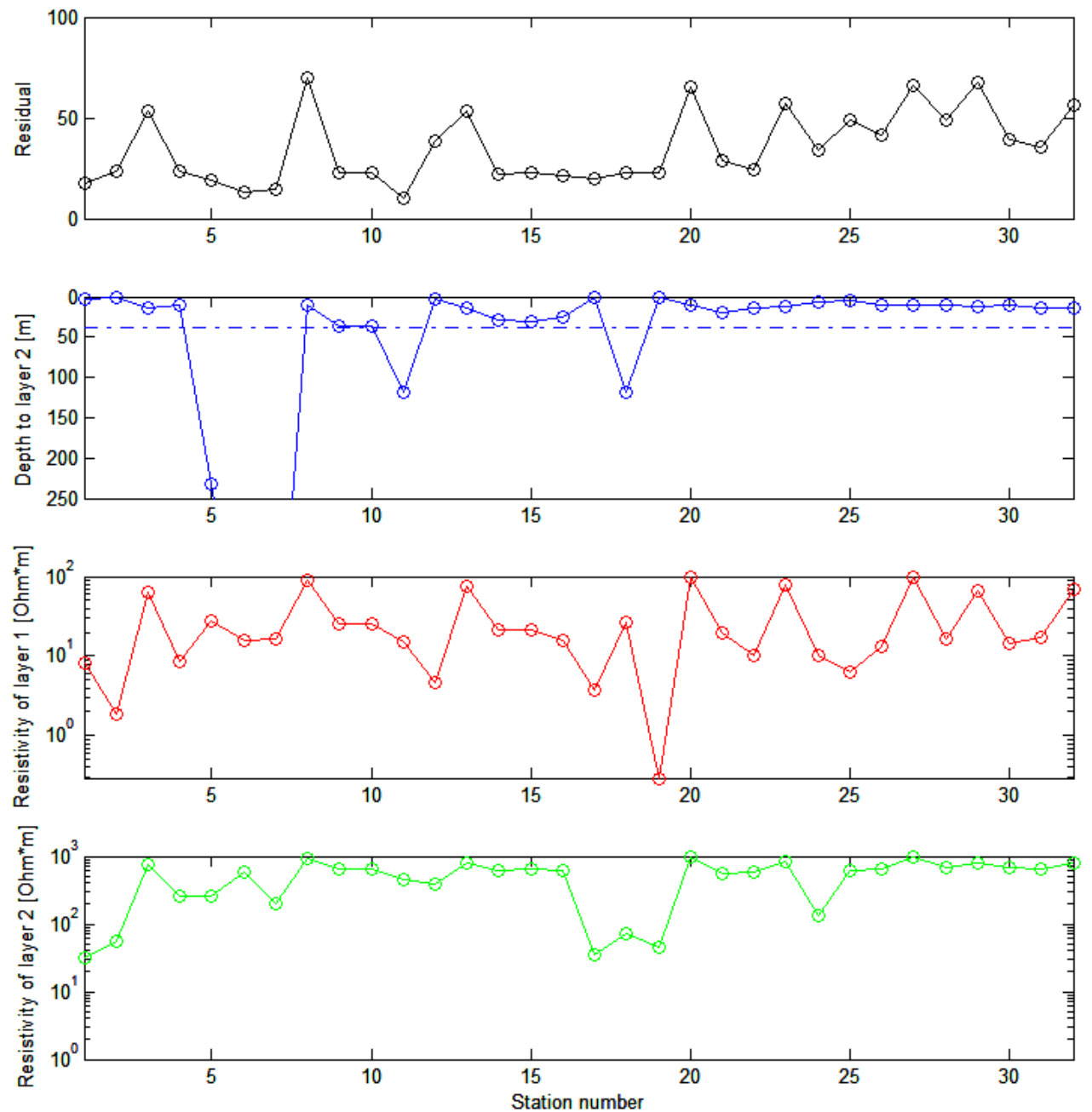

Figure 6.5. Inversion results using model 1. The dotted blue line on the second graph from the top represents the shallowest depth of penetration of the MaxMin system for the configuration used during the survey. 

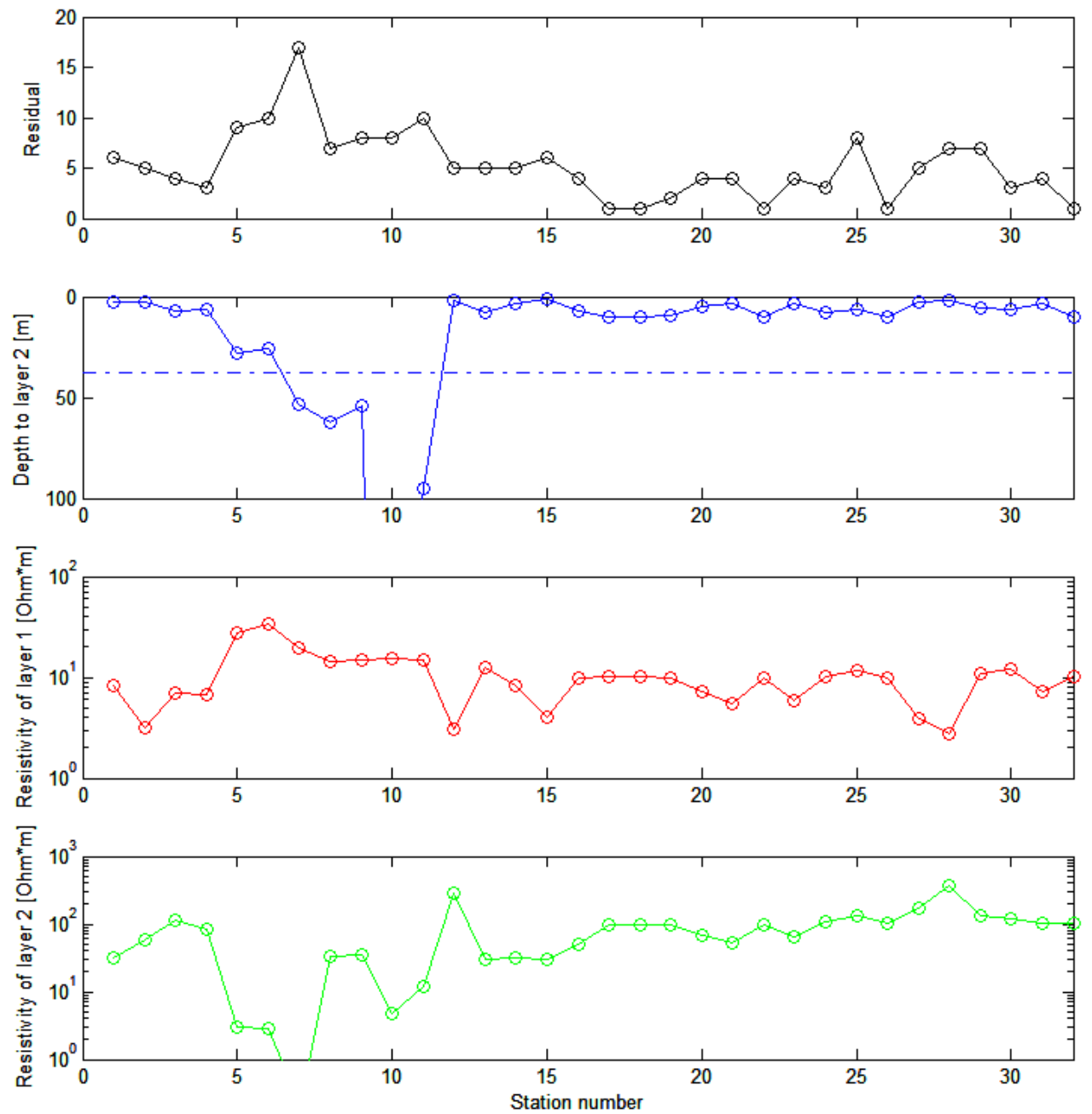

Figure 6.6. Inversion results using model 2. The dotted blue line on the second graph from the top represents the shallowest depth of penetration of the MaxMin system for the configuration used during the survey. 

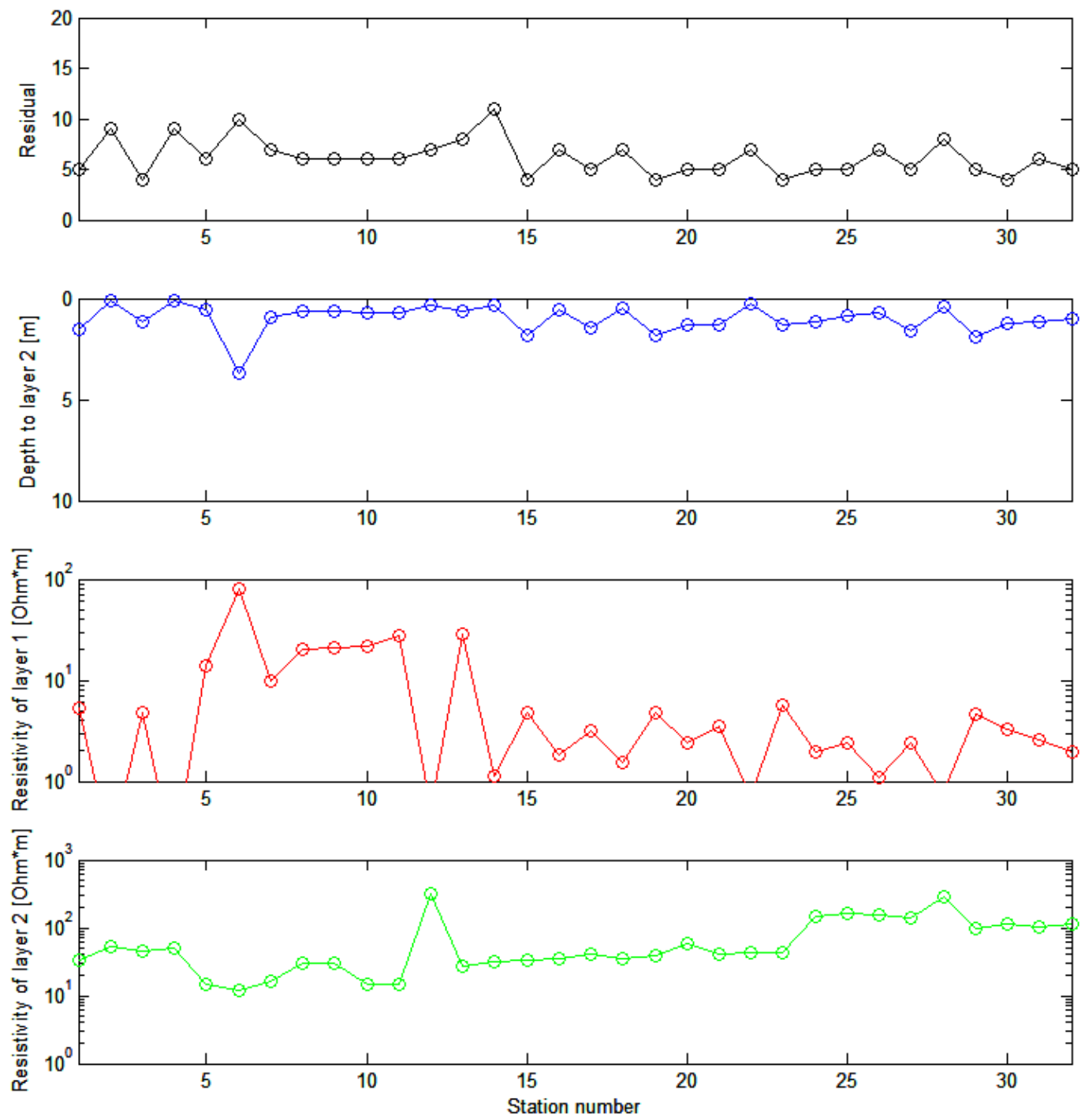

Figure 6.7. Inversion results using model 3. 


\subsubsection{Inversion of IMAGEM data}

Inversion of IMAGEM field data was performed on points A, B, and C of Figure

6.1. The response at point $A$ is caused by a man-made culvert buried at a depth of approximately $1 \mathrm{~m}$. Point $\mathrm{B}$ is background response not related to any anomalies. The response at point $\mathrm{C}$ is believed to correspond with a graphite deposit. The decay curves at these locations are presented in Figure 6.8. The inversion was performed using AarhusInv (Section 4). A summary of the initial guess models used at each point and the resulting inversion is presented in Table 6.2. For the two anomalies at points $A$ and $C$, a three-layer initial guess model was used, whereas a simpler two-layer initial guess model was used for the background response at point $B$. 


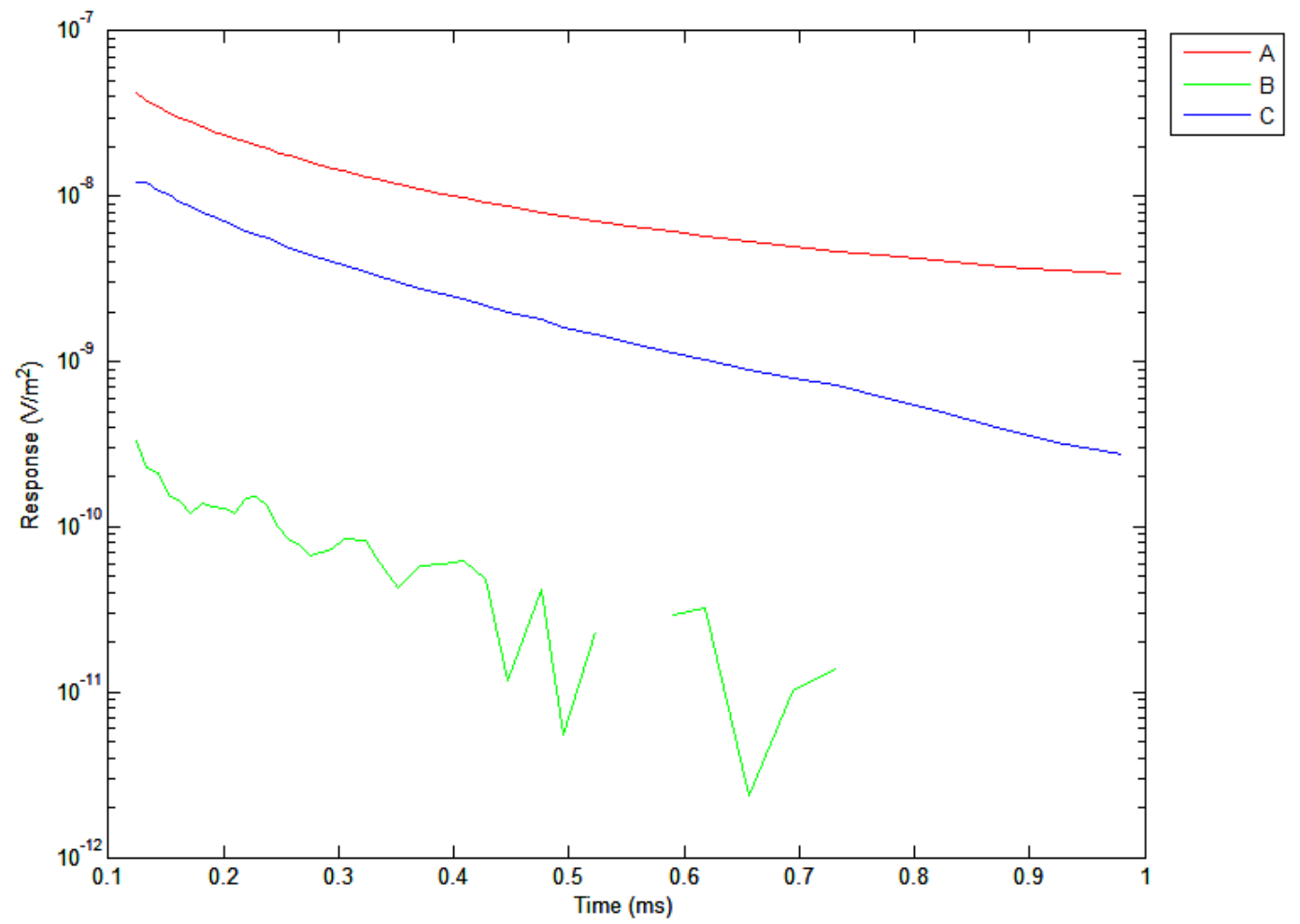

Figure 6.8. Decay curves of points A, B, and C of Figure 6.1. The gaps observed in curve B is caused by negative values which have been removed from analysis. 
Table 6.2. Summary of the results of inverting field data with the IMAGEM system.

\begin{tabular}{|c|c|c|c|}
\hline & \multicolumn{2}{|c|}{ Point A } \\
\hline & & Initial guess model & Inverted model \\
\hline \multirow{3}{*}{ Resistivity $(\Omega \cdot m)$} & Layer 1 & 100 & 182.4 \\
\hline & Layer 2 & 5 & 0.1 \\
\hline & Layer 3 & 1,000 & $1,137.2$ \\
\hline \multirow{3}{*}{ Thickness (m) } & Layer 1 & 1 & 17.1 \\
\hline & Layer 2 & 1 & 0.6 \\
\hline & Layer 3 & $\infty$ & $\infty$ \\
\hline \multicolumn{2}{|c|}{ \# of iterations } & $\mathrm{N} / \mathrm{A}$ & 9 \\
\hline \multicolumn{2}{|c|}{ Start residual } & $\mathrm{N} / \mathrm{A}$ & 70.026 \\
\hline \multicolumn{2}{|c|}{ End residual } & N/A & 12.108 \\
\hline & & \multicolumn{2}{|c|}{ Point B } \\
\hline & & Initial guess model & Inverted model \\
\hline \multirow{2}{*}{ Resistivity $(\Omega \cdot \mathrm{m})$} & Layer 1 & 100 & 20,000 \\
\hline & Layer 2 & 1,000 & 6.4 \\
\hline \multirow{2}{*}{ Thickness (m) } & Layer 1 & 10 & 90.1 \\
\hline & Layer 2 & $\infty$ & $\infty$ \\
\hline \multicolumn{2}{|c|}{ \# of iterations } & $\mathrm{N} / \mathrm{A}$ & 13 \\
\hline \multicolumn{2}{|c|}{ Start residual } & N/A & 59.839 \\
\hline \multicolumn{2}{|c|}{ End residual } & $\mathrm{N} / \mathrm{A}$ & 10.827 \\
\hline & & \multicolumn{2}{|c|}{ Point C } \\
\hline & & Initial guess model & Inverted model \\
\hline \multirow{3}{*}{ Resistivity $(\Omega \cdot m)$} & Layer 1 & 100 & $2,507.8$ \\
\hline & Layer 2 & 10 & 0.2 \\
\hline & Layer 3 & 1,000 & 410 \\
\hline \multirow{3}{*}{ Thickness (m) } & Layer 1 & 1 & 23.2 \\
\hline & Layer 2 & 1 & 439.5 \\
\hline & Layer 3 & $\infty$ & $\infty$ \\
\hline \multicolumn{2}{|c|}{ \# of iterations } & N/A & 17 \\
\hline \multicolumn{2}{|c|}{ Start residual } & $\mathrm{N} / \mathrm{A}$ & 140.403 \\
\hline \multicolumn{2}{|c|}{ End residual } & $\mathrm{N} / \mathrm{A}$ & 3.215 \\
\hline
\end{tabular}




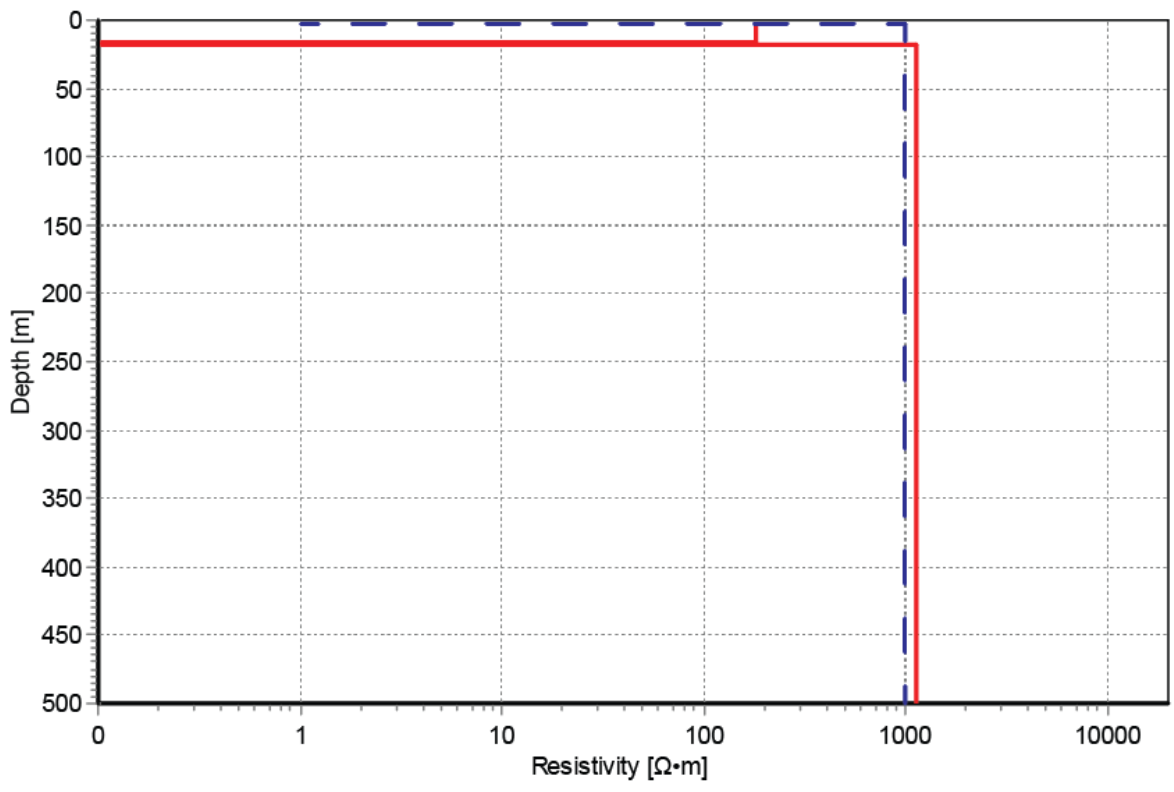

Inverted model

- - - Initial guess model

Figure 6.9. Inverted model at point A. 


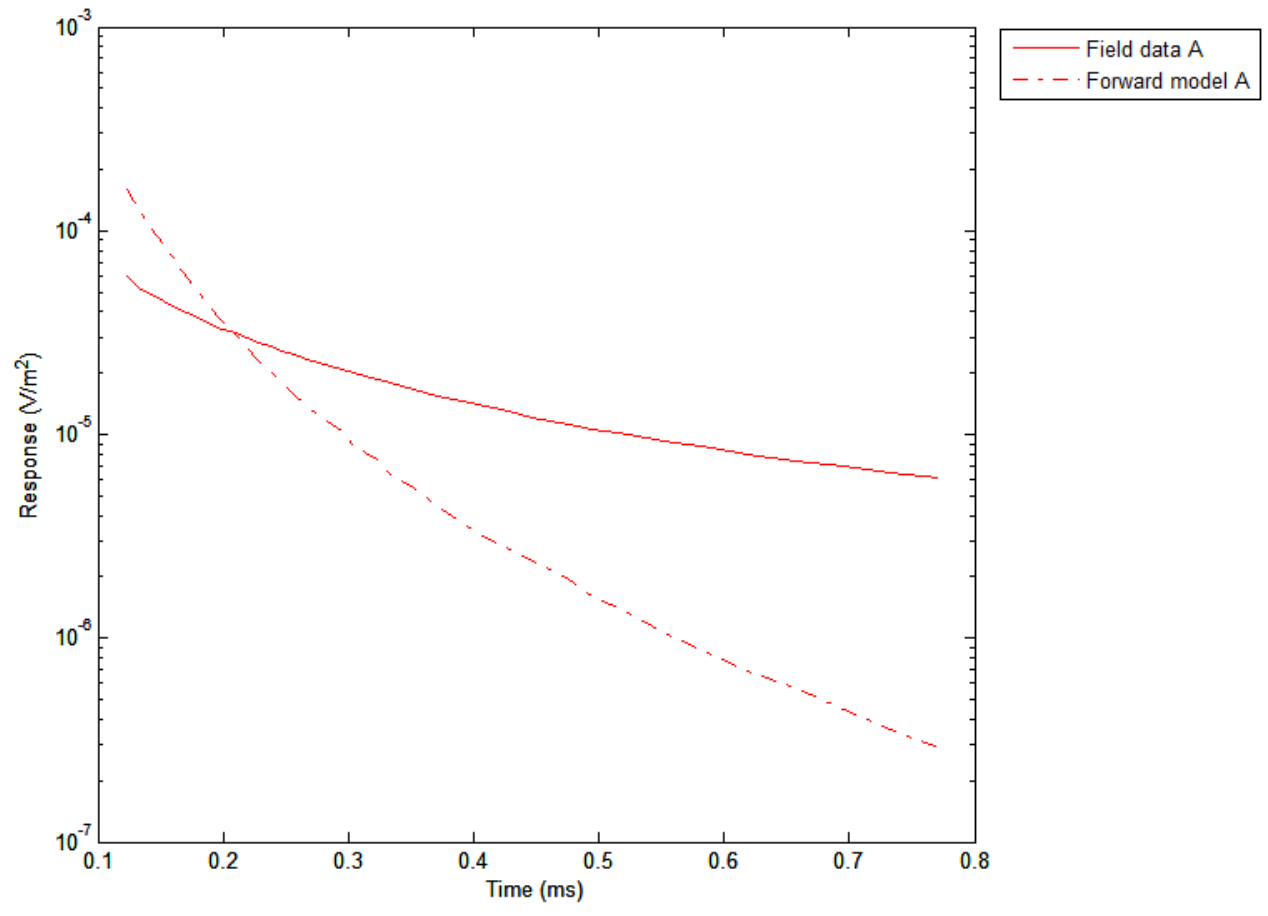

Figure 6.10. Comparing the IMAGEM field decay curve with the decay curve achieved by forward modelling the inverted model at point $A$. 


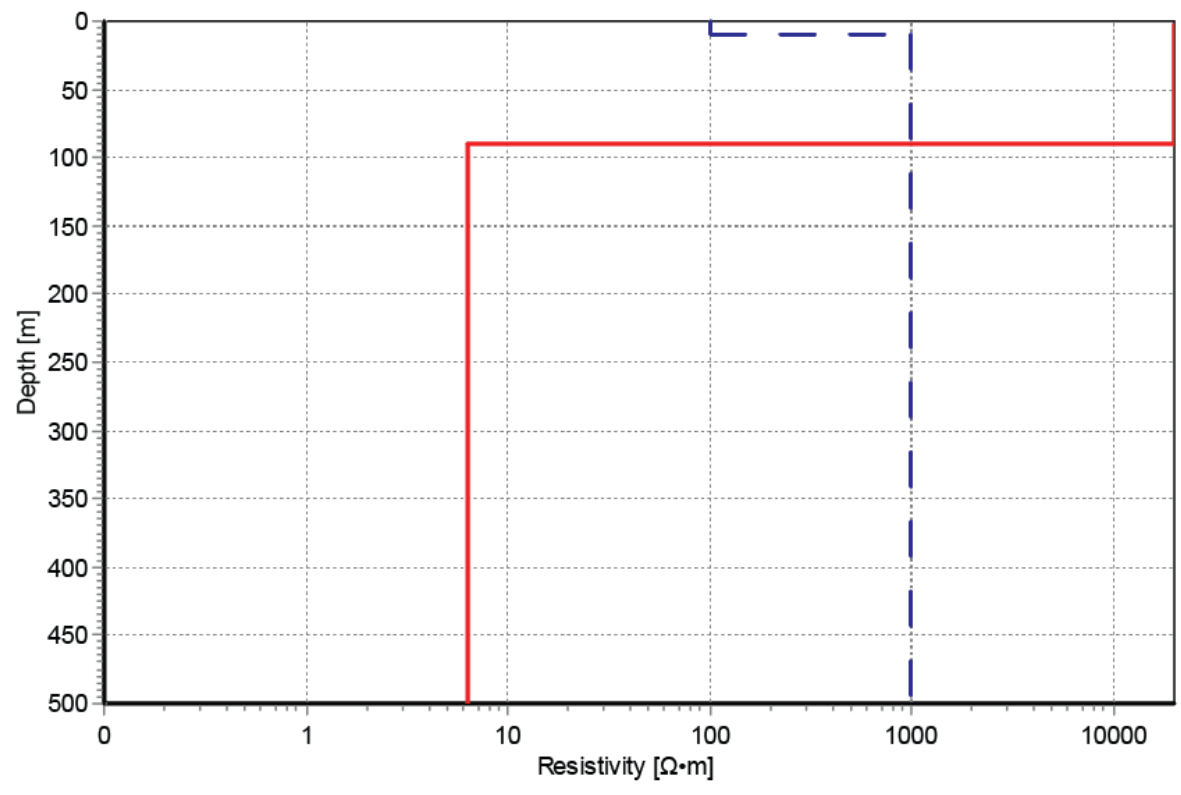

Inverted model

- - - Initial guess model

Figure 6.11. Inverted model at point B. 


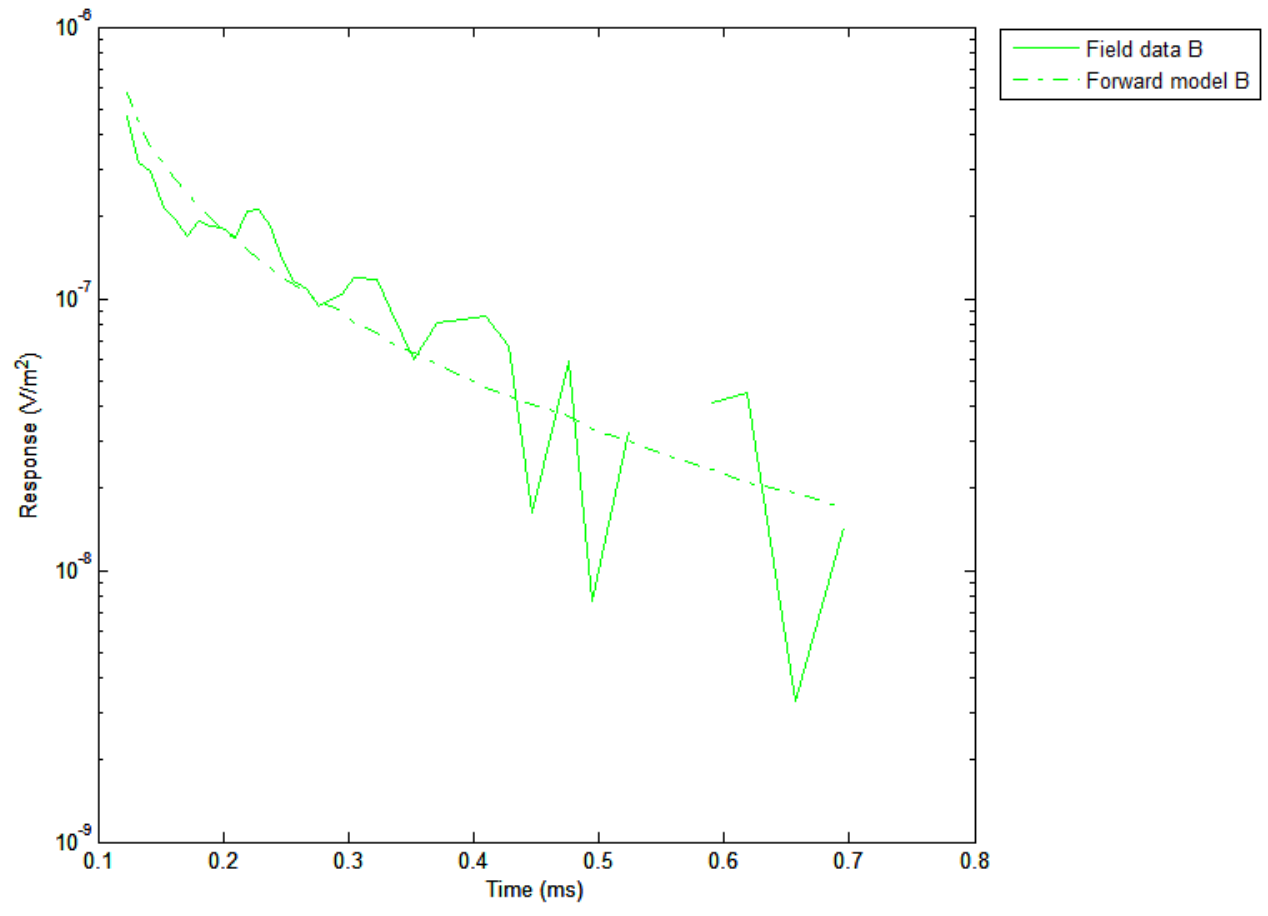

Figure 6.12. Comparing the IMAGEM field decay curve with the decay curve achieved by forward modelling the inverted model at point $B$. 


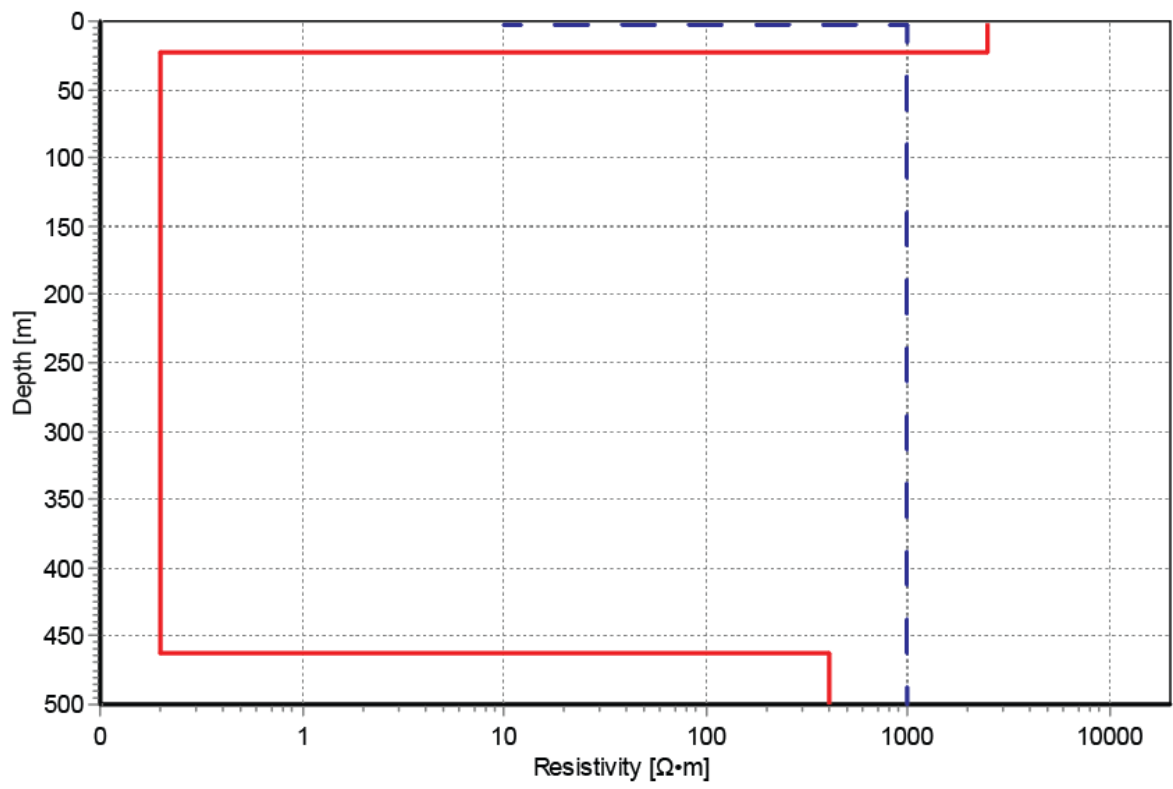

Inverted model

- - - Initial guess model

Figure 6.13. Inverted model at point C. 


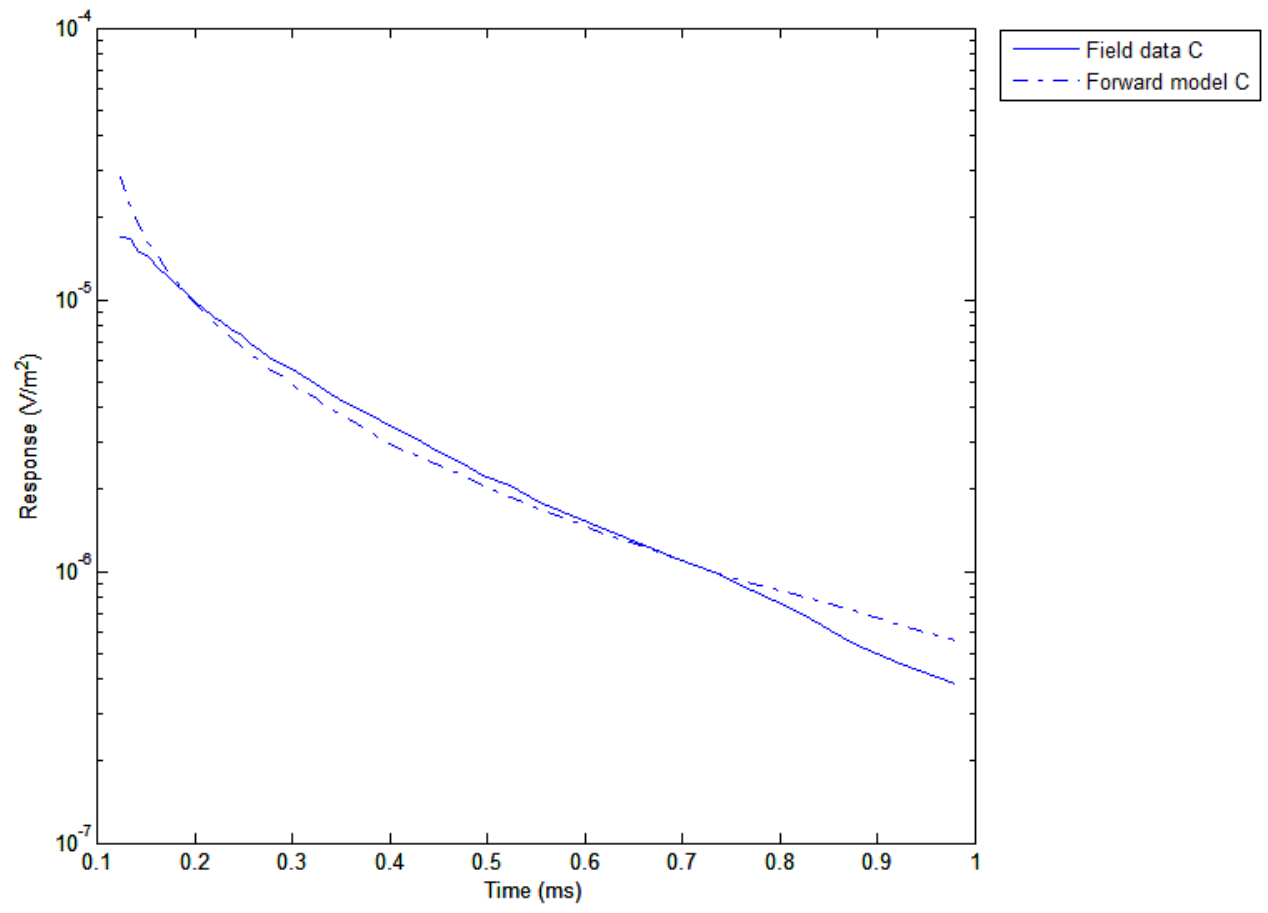

Figure 6.14. Comparing the IMAGEM field decay curve with the decay curve achieved by forward modelling the inverted model at point $\mathrm{C}$. 
The inverted model of point $A$ is presented in Figure 6.9. The inverted model consists of an overburden layer overlaying a very thin $(0.6 \mathrm{~m})$ and very conductive $(0.1$ $\Omega \cdot \mathrm{m})$ layer. Beneath is a resistive $(1,137.2 \Omega \cdot \mathrm{m})$ basement. The decay curves of the field data and the forward model of the inverted model is presented in Figure 6.10. The two curves do not match well. The forward model curve decay faster than the IMAGEM field data curve.

The inverted model at point $B$ is presented in Figure 6.11. The inverted model consists of a very thick $(90.1 \mathrm{~m})$ and very resistive $(20,000 \Omega \cdot \mathrm{m}$, which is the maximum allowed by the program's configuration file) layer overlaying a conductive $(6.4 \Omega \cdot \mathrm{m})$ half-space. The decay curves of the field data and the forward model of the inverted model are presented in Figure 6.12. Despite the high amount of noise, the curves match fairly well in terms of the general slope and amplitude.

The inverted model for point $C$ is presented in Figure 6.13. The inversion resulted in a model consisting of a resistive $(2,507.8 \Omega \cdot \mathrm{m})$ first layer overlaying a very thick (439.5 $\mathrm{m})$ and very conductive $(0.2 \Omega \cdot \mathrm{m})$ second layer, on top of a $410 \Omega \cdot \mathrm{m}$ half-space. The decay curves of the field data and the forward model of the inverted model is presented in Figure 6.14. The curves match quite well.

Each model converged after a reasonable number of iterations, and the end residuals for each inversion are a bit high, but not unreasonable (values smaller than 1.0 are good). Results, however, are questionable. At point $A$, the inversion did not resolve layer 2 (culvert) and exaggerated the thickness of layer 1 instead. The inverted model at 
point A can effectively be considered a 2 layer model, with resistivity values close to those of the initial guess model. The inverted model at point B reached the highest possible resistivity for the first layer allowed by the program. It is rather disconcerting that the inversion suggested a resistive near-surface layer over a conductive basement whereas the input model (which was chosen to reflect known geology) consisted in a conductive near-surface layer over a resistive basement. The interface between layers 1 and 2 is at $90.1 \mathrm{~m}$, a depth much greater than the depth of investigation of the IMAGEM system. The model at point $C$ has similar problems as the model at point $B$, with the interface between layers 2 and 3 located at an unrealistically large depth.

The inversion exercise did not lead to realistic results. The unconventional transmitter design of the IMAGEM system probably cannot be modelled as a simple loop (Figure 4.4). Further research is needed to understand the system's signal better. Processing steps such as deconvolution might need to be applied to the field data before inversion is attempted. However, for points $A$ and $C$, the problem may also stem from improper use of $1 D$ inversion on $2 D / 3 D$ structures. The man-made culvert and the laterally thin graphite deposit are small enough, relative to the footprint of the system, that any measurement made by the IMAGEM system is also affected by the adjacent material, which has a different resistivity. Thus, a 1D inversion would not take into account this surrounding material, and would therefore result in an incorrect inversion. Furthermore, for point $A$, the decay curve caused by the man-made culvert cannot be described by a decaying slope of $t^{-5 / 2}$, as is used in the AarhusInv software, because of its 
extreme conductivity. This is one of the reasons why the software could not match the decay curve of the field data.

\subsection{Matawinie}

The IMAGEM system was used to survey a graphite-hosting property in the Grenville Province of the Canadian Precambrian Shield, in the Matawinie region of Quebec, located $140 \mathrm{~km}$ north of the Port of Montreal. This region is especially prospective for graphite because it hosts one of only two graphite-producing mines in Canada. The Matawinie property is owned by Nouveau Monde (Dubé \& Desaulniers, 2015). The graphite deposit is hosted in proterozoic crystalline marble and graphitic paragneiss. This is underlain by paleoproterozoic and Mesoproterozoic syenite, monzonite, monzodiorite and other related alkaline intrusions (Figure 6.15).

The survey data was interpolated using the Inverse Distance Weighing (IDW) method. This method calculates the value of a point based on the values of other data points nearby using

$$
\hat{v}=\frac{\sum_{i=1}^{n} \frac{1}{d^{p_{i}}} v_{i}}{\sum_{i=1}^{n} \frac{1}{d^{p_{i}}}}
$$

where $d$ is the distance of the known value $(v)$ from the unknown value to be calculated $(\hat{v}), p$ is the power, in this case 2 , and $n$ is the number of known data points. The interpolation must be kept to a small fixed radius because of the high spatial resolution of the system. 
The result of channel 50 of the survey for the western zone is presented in Figure 6.16. This channel was chosen as it is a mid-time channel, thus has a good signal-tonoise ratio and is unaffected by early-time channel effects. There are multiple areas of high conductance scattered throughout the survey lines, but with most concentrated on the line striking SE-NW. There are two anomalies near the western edge of the survey, and two or three more near the centre on this SE-NW line. Two other small anomalies are located just south of this, on the NE-SW striking lines. The anomaly on the eastern edge of the survey is located on a public road and may be caused by a passing vehicle.

The survey is visualized with increasing depth in Figure 6.17, by presenting the interpolation from early time channel (30) to late time channel (90) stacked vertically, with the early time being on top. The signal-to-noise ratio visibly decreases with increasing channel times, as is apparent by the discoloring of the background response; the amplitude of the background response and the anomalies are more similar at channel 90 than at channel 30. The western anomaly pinches out towards the east with depth, and is no longer visible at channel 90. In contrast, a small anomaly near-surface located just east of the latter increases in size with depth. The same is seen for another anomaly on the same line, located near the centre of the survey area. The small anomalies located on the NE-SW lines also increase in size with depth.

The anomalies observed are approximately on the order of 1-100 metres in size. The scattered nature observed would suggest that the deposit is formed of many small clusters of graphite. 


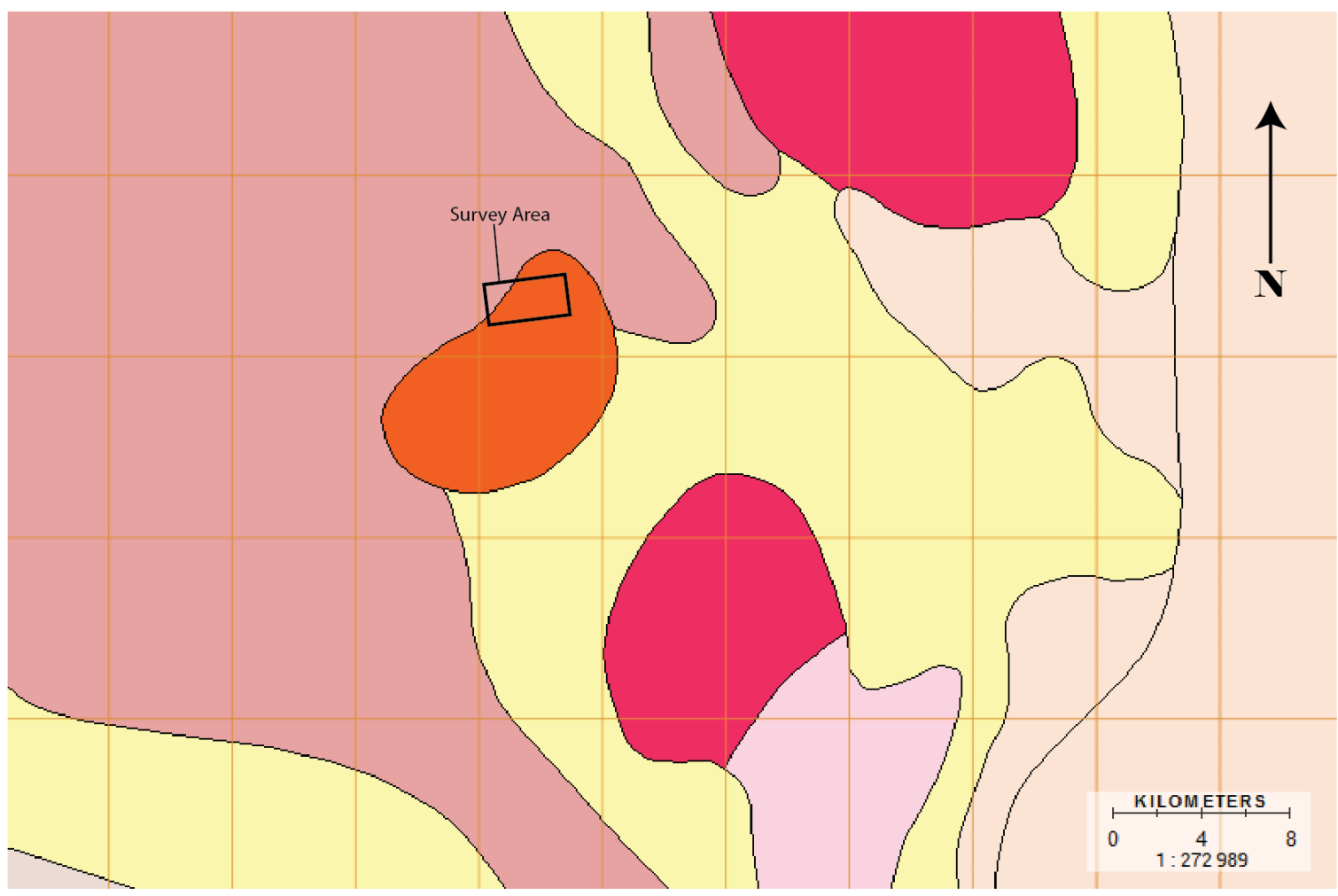

PROTEROZOIC

Paragneiss, granulitic intrusive rocks and felsic metavolcanic rocks (Chain Lakes Massif)
PALEOPROTEROZOIC AND MESOPROTEROZOIC GRANITOIDS

Granite, quartz monzonite, quartz syenite and pegmatite

Syenite, monzonite, monzodiorite and other related alkaline intrusions

Orthopyroxene-bearing granitoids: charnockite, mangerite, jotunite and hypersthene syenite

Unsubdivided granitoids

Migmatite

Figure 6.15. Local geology of the Matawinie survey area (source: Geological Survey of Quebec). 


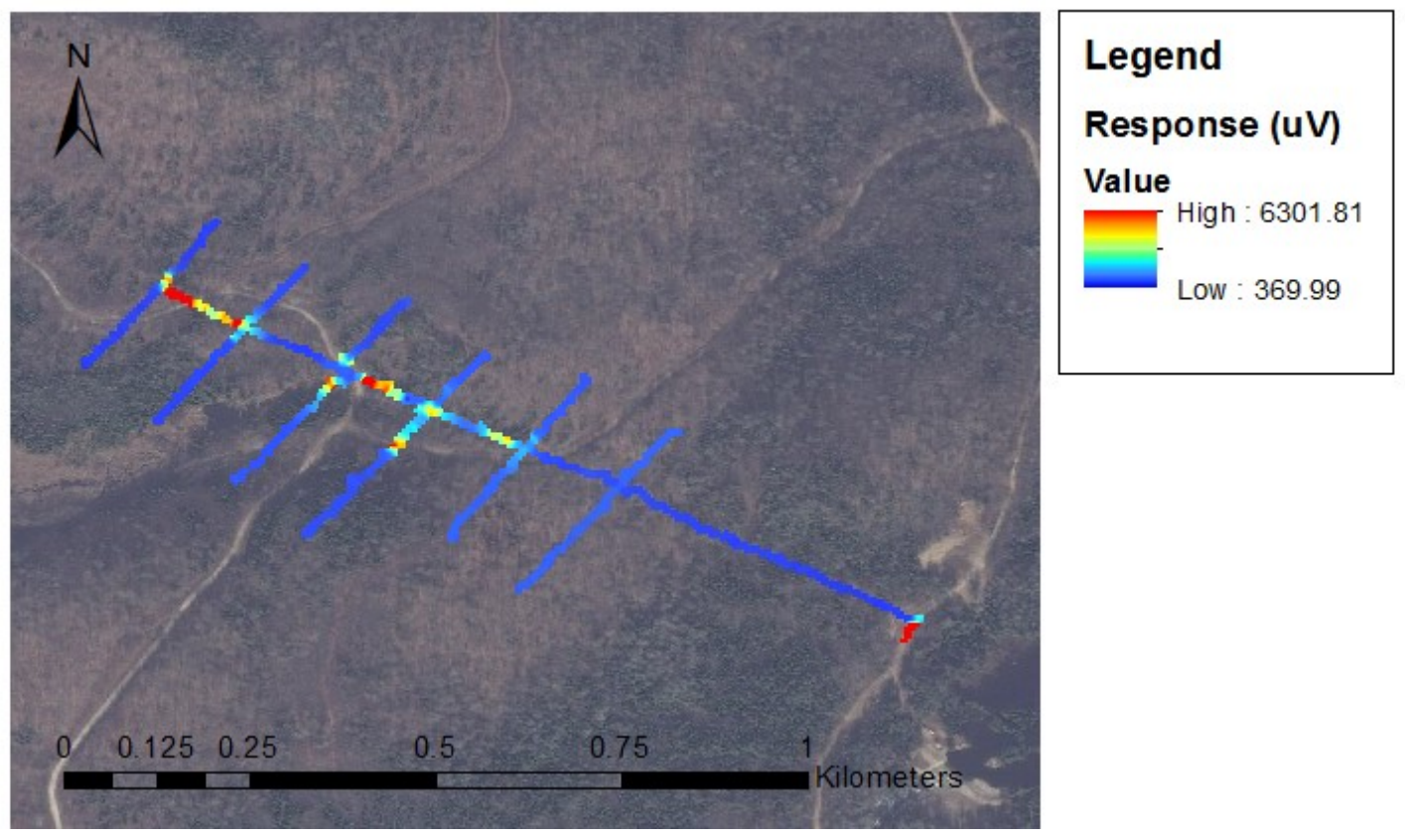

Figure 6.16. Interpolated results of the Matawinie survey for channel 50 using Inversed Distance Weighing (IDW). (Data source: Nouveau Monde Mining Inc., shown with permission). 


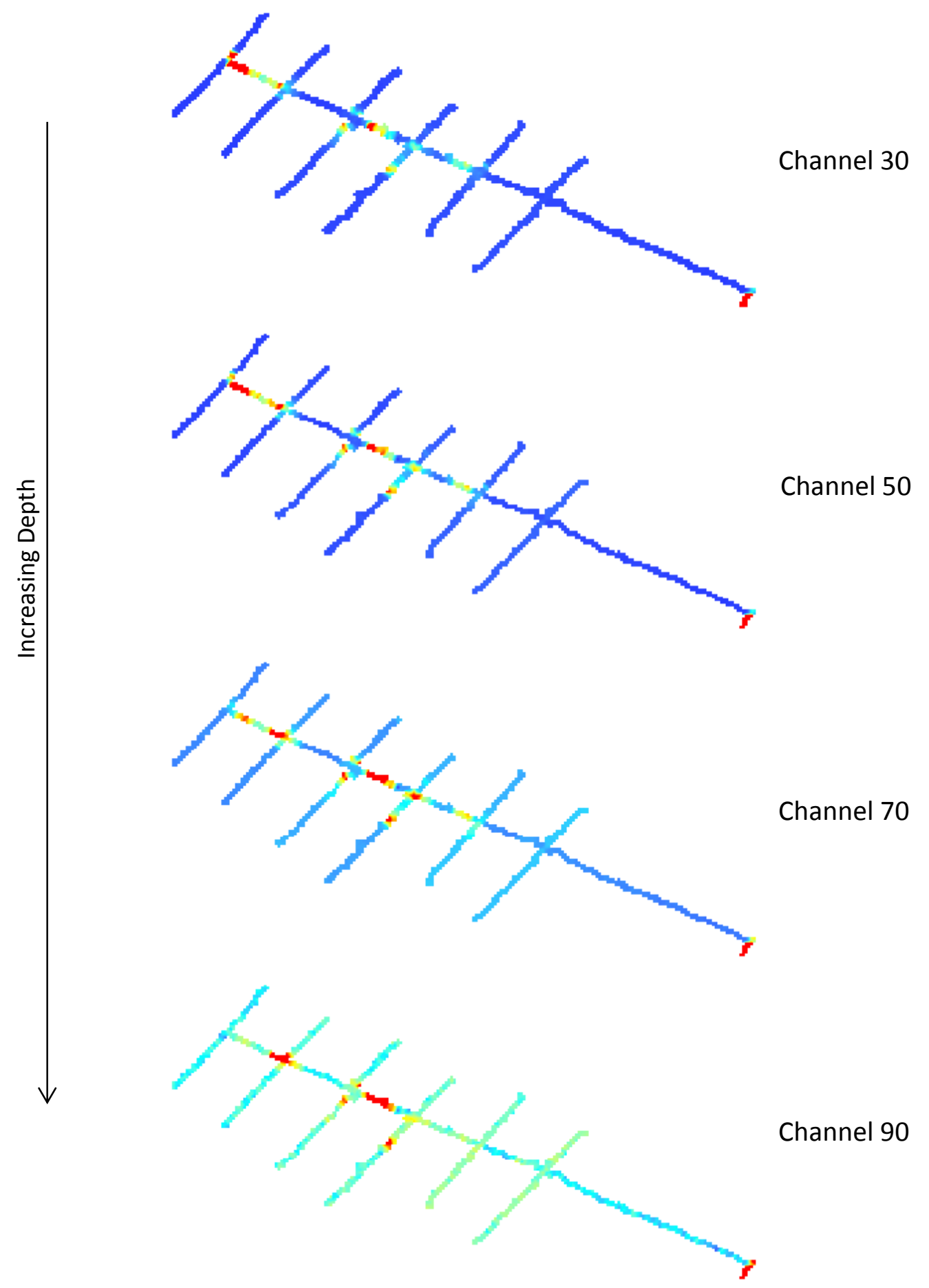

Figure 6.17. Visualizing survey data at increasing depth using the interpolated maps at increasing channel times. 


\subsection{Lac Tétépisca}

The survey was conducted in Lac Tétépisca, approximately $240 \mathrm{~km}$ north of BaieComeau, Quebec, in the Grenville Province of the Canadian Precambrian Shield. The survey covered $7.5 \mathrm{~km}^{2}$ of rough topography terrain with a line spacing of $100 \mathrm{~m}$ (Figure 6.18). A MaxMin survey was conducted in parallel with IMAGEM in some areas of the survey grid to serve as a basis for comparison. Both the MaxMin and IMAGEM systems achieved a similar daily production of $\sim 4$ line-km (Ricard et al., 2015).

Figure 6.19 shows IMAGEM channel 50 data in map-view. Such a display, which can readily be done with minimal processing by the field operator, allows quality control of the data to be done by a quick visual inspection and the ground data to be compared with airborne data. The display reveals high amplitude anomalies which are consistent on adjacent survey lines, such as the elongated $200 \mathrm{~m}$-long and $150 \mathrm{~m}$-wide NE-SW trending zone in the northeast part of the survey area.

Figure 6.20 shows coincident IMAGEM and MaxMin data for a 900 m-long survey line. The transmitter-receiver separation of the MaxMin survey was $50 \mathrm{~m}$. MaxMin data were recorded at $220,880,3520$, and $14080 \mathrm{~Hz}$. Between stations $-100 \mathrm{~m}$ and $-200 \mathrm{~m}$ in Figure 6.20, the MaxMin data features a prominent $110 \mathrm{~m}$ wide anomaly corresponding to a $60 \mathrm{~m}$ thick body or multiple sub-parallel closely spaced bodies. An average conductivity-thickness of 37 Siemens was calculated for this anomaly, which corresponds to a good conductor (Ricard et al., 2015). Interestingly, the high spatial resolution of the IMAGEM system allowed it to resolve two anomalies, likely to be 
associated with two distinct thinner bodies, in the same area. Between stations $-550 \mathrm{~m}$ and $-850 \mathrm{~m}$ in Figure 6.20 , the MaxMin data has a very complicated signature, which confuses interpretation. This is probably due to topography and presence of highly magnetic horizon. This part of the line features rapid elevation changes, as seen on the elevation profile (Figure 6.18). The MaxMin system, especially its in-phase component, is sensitive to topography. It is difficult for operators in the field to orient the coils to achieve a perfect co-planar configuration between the MaxMin transmitter and receiver coils in rugged terrain. The IMAGEM system, with its rigid geometry, does not suffer from these problems. Its data in the same area shows several well-defined prominent peaks. 


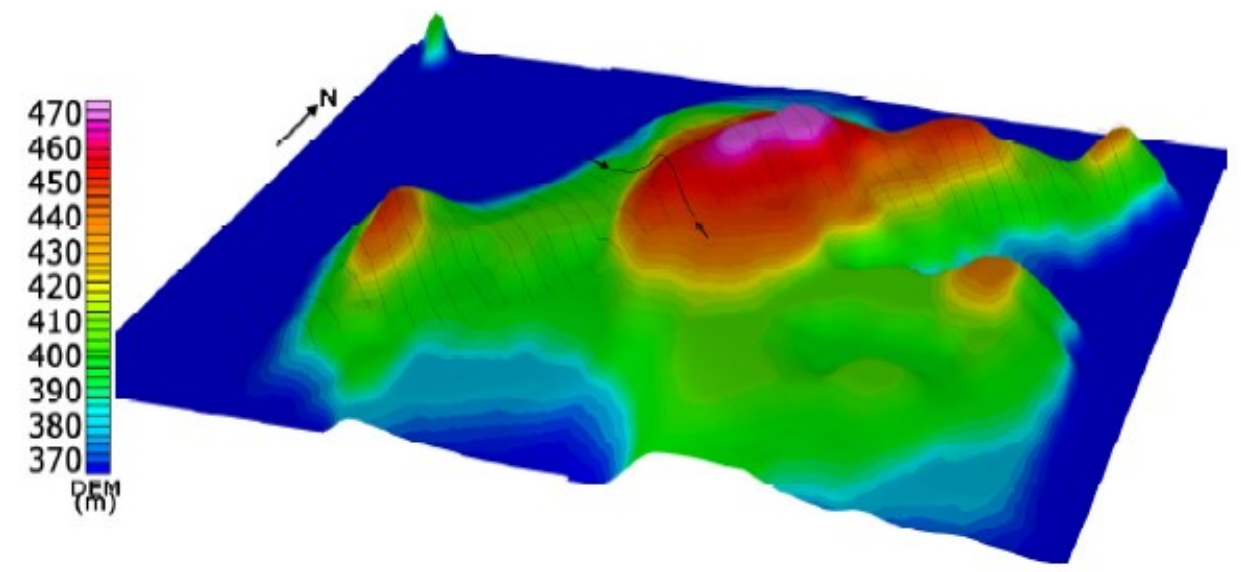

Figure 6.18. Digital elevation model (DEM) and survey lines for the northern part of the survey area (Ricard et al., 2015). Arrows point to the line presented in Figure 6.19. 


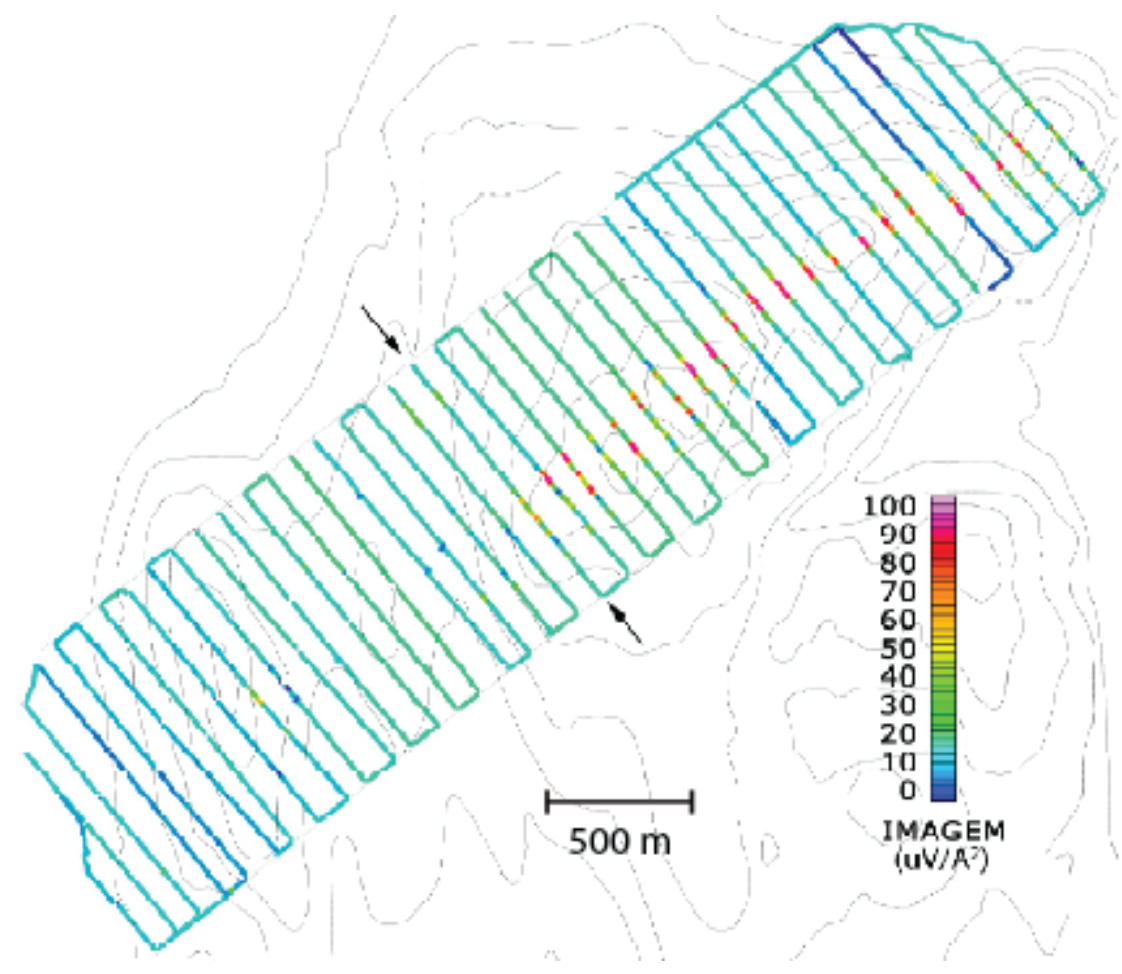

Figure 6.19. IMAGEM data results. Amplitude of channel 50 ( $0.3 \mathrm{~ms}$ after turn-off) color coded against elevation contours (Ricard et al., 2015). Arrows point to the line presented in Figure 6.18 (Data source: Focus Graphite Inc., shown with permission) 


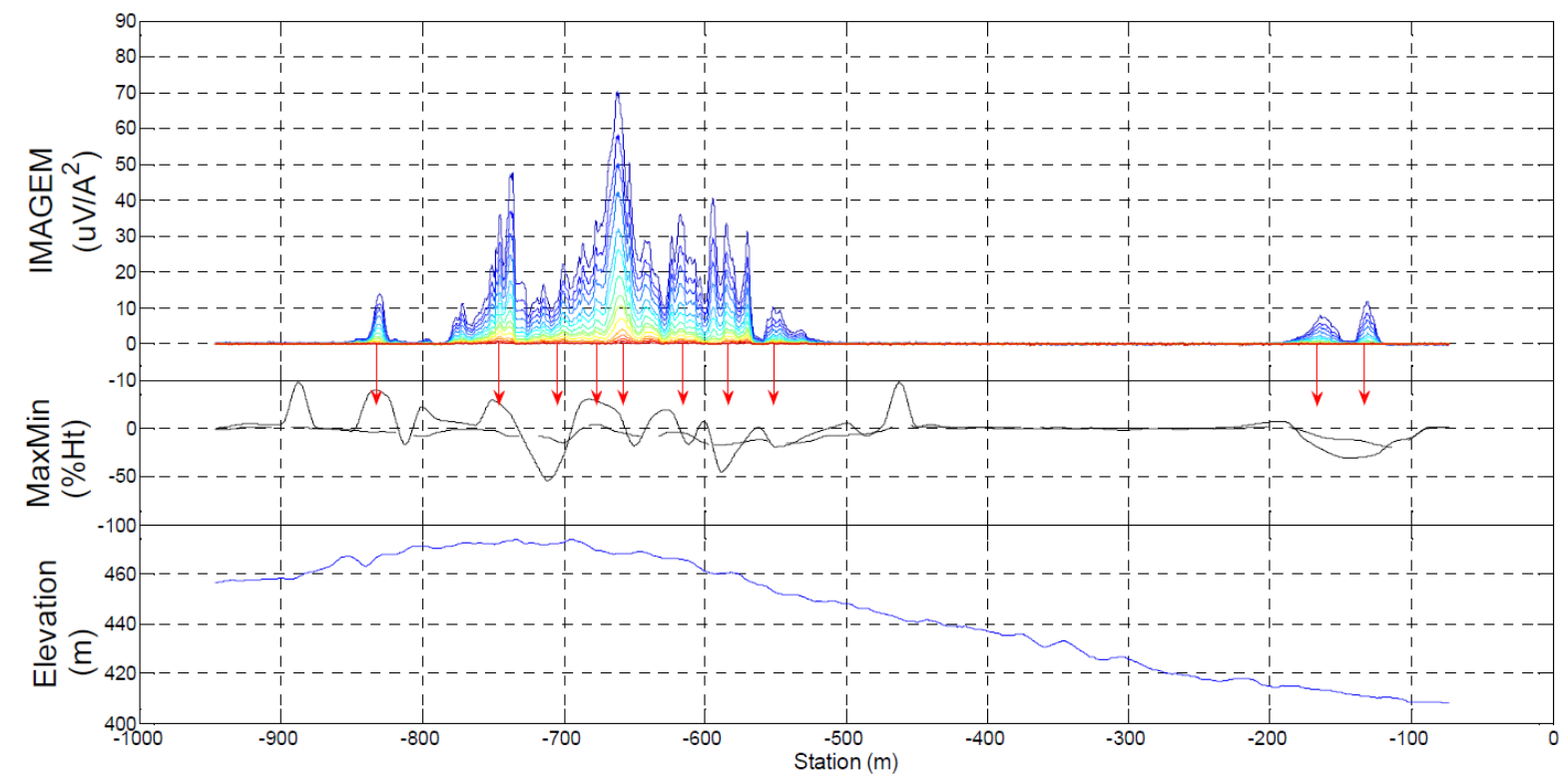

Figure 6.20. IMAGEM data for channels 45 to 90 ( $0.21 \mathrm{~ms}$ to $4.3 \mathrm{~ms}$ after turn off; top panel). MaxMin data at a frequency of $3520 \mathrm{~Hz}$ (middle panel: solid and dotted lines are the in-phase and quadrature components, respectively), and elevation profile (bottom panel) (Ricard et al., 2015). 


\section{Discussion and summary}

The results of forward modeling presented in Section 5.1, specifically Figure 5.6 to Figure 5.10, shows the effects of very highly conductive environments, suggesting the use of caution when surveying in highly-conductive environments. The results showed that there exists a 'blinding' effect caused by very conductive near-surface layers which will mask the measurements of any other layers beneath, similar to a bright light being flashed into the eyes; it is difficult or impossible to see beyond the light. Even using a graphite layer with a conductivity of $10 \Omega \cdot m$, this effect is still observed, although only at the earliest (0.001-0.06 ms after turn-off) time channels. Mid-time channels (0.3-1 ms after turn-off) may be more reliable, as they are still early enough to be above the noise and still evade this blinding effect caused by very conductive layers. Nonetheless, this effect, where the response becomes insensitive to further increases in the thickness of the conductive layer, in turn causes problems during inversion by making it impossible to resolve deeper layers.

The inversion of synthetic data in Section 5.2 demonstrated that the inversion capabilities of the AarhusInv program are working properly when modelling the IMAGEM system. Even for decay curves with a relatively low signal to noise ratio (lower than what is observed in the IMAGEM's field data) the software was able to properly invert the data. This is useful to know, as it helps isolate the problem encountered when inverting field data in Section 6.1.2.2. It shows that these problems are not caused by noisy data. 
The case studies of Chapter 6 showcased the high spatial resolution of the IMAGEM system, especially when compared to the MaxMin system. For the Mayo survey (Section 6.1) the IMAGEM system was able to clearly differentiate two anomalies (Figure 6.1), whereas for the MaxMin, only one anomaly is observed. This is again seen in Section 6.3; looking at one line of the survey, the IMAGEM system could differentiate two anomalies whereas only one could be seen with the MaxMin (Figure 6.20).

The inversion of field data with AarhusInv was problematic both for IMAGEM and the MaxMin systems. The MaxMin system surveys at discrete stations and, with 3 sounding frequencies, there are only 6 data points available for inversion. The problem is ill-posed; the input data does not provide enough constraints. A sign of this is that a small change in the parameters of the initial guess model produces a significant change in the inverted model. Perhaps the largest contributor to the problematic inversion results was the use of $1 \mathrm{D}$ inversion for 2D or 3D targets. However, the inversion of point $B$ (Figure 6.11, Figure 6.12) is a 1D situation, as there is no rapid change in subsurface resistivity measured within the footprint of the system. Thus, the problematic inversion results, in this case, are not solely caused by the use of $1 D$ inversion in 2D/3D situations.

The inversion results of the IMAGEM system were for the most part geologically unreasonable. Causes might be in hardware and/or software. The decay curves of the IMAGEM system, when compared to the decay curves generated by AarhusInv while modelling the IMAGEM for a plausible geological model that is representative of the geology of the region, were very different in shape. Either further refinements of the 
transmitter and receivers are needed, or the shape of the decay curve needs to be "conditioned" through post-processing before inversion is attempted. With its great system configuration flexibility, AarhusInv remains the best program to model the IMAGEM system.

The IMAGEM system is still in development and is constantly being improved. Its main strengths are its high spatial resolution and its sensitivity to strong near-surface conductors. It already shows great potential as a tool to detect and delineate small graphite deposits. 


\section{Bibliography}

Apex Parametrics Limited, n.d. Apex parametrics MaxMin I-8 system. Ontario: s.n.

Attwa, M., Gunther, T., Grinat, M. \& Binot, F., 2011. Evaluation of DC, FDEM and IP resistivity methods for imaging perched saltwater and a shallow channel witihin coastal tidal flat sediments. Journal of Applied Geophysics 75, pp. 656-670.

Auken, E. et al., 2005. Piecewise 1D laterally constrained inversion of resistivity data. Geophysical Prospecting, pp. 497-506.

Auken, E. et al., 2014. An overbiew of a highly versatile forward and stable inverse algorithm for airborne, ground-based and borehole electromagnetic and electric data. Exploration Geophysics, pp. 1-13.

Burger, H. R., Sheehan, A. F. \& Jones, C. H., 2006. Introduction to applied geophysics. W. W. Norton \& Company, Volume 1, pp. 499-505.

Christiansen, A. V. \& Auken, E., 2008. Presenting a free, highly flexible inversion code. s.I., Society of Exploration Geophysicists, p. 4.

Cunningham, M., 2013. Real-time signal discrimination and audio feedback for a new ground time-domain electromagnetic survey system.. B.Sc. Thesis, Department of Earth Sciences, Carleton University.

Dubé, J. \& Desaulniers, E., 2015. Geophysical approach for graphite exploration: The Matawinie example in Quebec. Toronto, Canadian Exploration Geophysical Society.

Frischknecht, F. C., Labson, V. F., Spies, B. R. \& Anderson, W. L., 1991. Electromagnetic Methods in Applied Geophysics. Society of Exploration Geophysicists, Volume 2, pp. 258-259.

Grant, F. S. \& West, G. F., 1965. Interpretation theory in applied geophysics. McGraw-Hill Book Company, Volume 1, pp. 444-464.

Gwinn, G. R., 1943. Graphite - Natural and Manufactured. United States Department of interior.

Kalyoncu, R. S., 2000. Graphite. USGS Minerals commodity.

Krauss, U. H., Schmidt, H. W., Taylor, Jr., H. A. \& Sutphin, D. M., 1998. International strategic minerals inventory summary report -- Natural graphite.. U.S. Geological Survey Circular, Issue 930-H, pp. 1-17.

Mackinnon, A. \& LeBaron, P. S., 1992. Graphite Occurences of the Frontenac Axis, Earth Ontario. Ontario Geological Survey, Mineral Deposits Circular, Volume 33, pp. 3-31. 
Nabighian, M. N. \& Macnae, J. C., 1991. Electromagnetic methods in applied geophysics. Society of Exploration Geophysicists, Volume 2, pp. 427-431.

Olson, D. W., 2012. Graphite. USGS 2012 Minerals Yearbook.

Ricard, J.-C., 2013. Développement d'un système émetteur-récepteur à géométrie fixe pour levés géophysiques à impulsion électromagnétique. M.A.Sc. Thesis, Department of eletrical engineering, University of Ottawa.

Ricard, J.-C., Meunier, E., Samson, C. \& Boivin, M., 2015. Graphite exploration with a new highresolution ground-based time-domain EM system: a case study from Lac Tétépisca, QC.. Toronto, Canadian Exploration Geophysical Society.

Simandl, G. J. \& Kenan, W. M., 1997. Crystalline flake graphite. British Columbia Geological Survey, pp. 1-3.

Telford, W. M., Geldart, L. P. \& Sheriff, R. E., 1990. Applied Geophysics. Press Syndicate of the University of Cambridge, Volume 2, p. 372.

U.S. Department of the Interior, 2015. Mineral commodity summaries 2015, s.I.: U.S. Geological Survey. 